\title{
KLT VARIETIES WITH TRIVIAL CANONICAL CLASS. HOLONOMY, DIFFERENTIAL FORMS, AND FUNDAMENTAL GROUPS
}

\author{
DANIEL GREB, HENRI GUENANCIA, AND STEFAN KEBEKUS
}

\begin{abstract}
We investigate the holonomy group of singular Kähler-Einstein metrics on klt varieties with numerically trivial canonical divisor. Finiteness of the number of connected components, a Bochner principle for holomorphic tensors, and a connection between irreducibility of holonomy representations and stability of the tangent sheaf are established. As a consequence, known decompositions for tangent sheaves of varieties with trivial canonical divisor are refined. In particular, we show that up to finite quasi-étale covers, varieties with strongly stable tangent sheaf are either Calabi-Yau or irreducible holomorphic symplectic. These results form one building block for Höring-Peternell's recent proof of a singular version of the Beauville-Bogomolov Decomposition Theorem.
\end{abstract}

\section{CONTENTS}

1. Introduction

\section{Part I. Preparations}

2. Notation and conventions $\quad 7$

3. Singular Kähler-Einstein metrics 11

4. The standard setting 14

$\begin{array}{ll}\text { Part II. Holonomy } & 18\end{array}$

5. The classification of restricted holonomy 18

6. The canonical decomposition of the tangent sheaf 19

7. Covering constructions 22

Part III. The Bochner principle on singular spaces 29

8. The Bochner principle for reflexive tensors and bundles 29

9. Proof of Theorem 8.1 ("Bochner principle for bundles") 31

10. Proof of Theorem 8.2 ("Bochner principle for tensors") 38

11. Augmented irregularity revisited 39

Part IV. Varieties with strongly stable tangent sheaf $\quad 41$

12. The basic dichotomy: CY and IHS 41

13. Fundamental groups 46

14. Examples 49

References $\quad 53$

Date: 16 th June 2020.

2010 Mathematics Subject Classification. 14J32, 14E30, 32J27.

Key words and phrases. varieties with trivial canonical divisor, klt singularities, Kähler-Einstein metrics, stability, holonomy groups, Bochner principle, irreducible holomorphic symplectic varieties, Calabi-Yau varieties, differential forms, fundamental groups, decomposition theorem.

Daniel Greb is partially supported by the DFG-Collaborative Research Center SFB/TRR 45. Henri Guenancia is partially supported by the NSF Grant DMS-1510214. Stefan Kebekus gratefully acknowledges partial support through a joint fellowship of the Freiburg Institute of Advanced Studies (FRIAS) and the University of Strasbourg Institute for Advanced Study (USIAS). 


\section{INTRODUCTION}

The structure of compact Kähler manifolds with vanishing first Chern class is encapsulated in the following fundamental result.

Theorem 1.1 (Decomposition Theorem, [Bea83b] and references therein). Let $X$ be a compact Kähler manifold with $c_{1}(X)=0 \in H^{2}(X, \mathbb{R})$. Then, there exists a finite étale cover $\widetilde{X} \rightarrow X$ such that $\widetilde{X}$ decomposes as a Kähler manifold as follows,

$$
\widetilde{X}=T \times \prod_{i} Y_{i} \times \prod_{j} Z_{j},
$$

where $T$ is a complex torus, and where the $Y_{i}\left(\right.$ resp. $\left.Z_{j}\right)$ are irreducible and simply connected Calabi-Yau manifolds (resp. holomorphic symplectic manifolds).

In view of a desired birational classification of varieties with Kodaira dimension zero and in view of the recent progress in the Minimal Model Program, it is important to extend the Decomposition Theorem, mutatis mutandis, to the setting of varieties with mild singularities. This turns out to be a very difficult challenge. Indeed, the strategy of the proof of Theorem 1.1 consists in first using Yau's solution to the Calabi conjecture in order to equip $X$ with a Ricci-flat Kähler metric, and then applying the deep theorems of De Rham and Cheeger-Gromoll to split a finite étale cover of $X$ according to its holonomy decomposition. The identification of the factors then follows from the Berger-Simons classification of holonomy groups combined with the Bochner principle, which states that holomorphic tensors are parallel.

Now, if $X$ is a singular projective variety with klt singularities and numerically trivial canonical divisor, one can still achieve the first step. More precisely, Eyssidieux, Guedj, and Zeriahi constructed "natural" Ricci-flat Kähler metrics $\omega$ on the regular locus $X_{\text {reg }}$ of $X$, [EGZ09]; see Section 3 for further references. However, as we will see in Proposition 4.2, the Kähler manifold $\left(X_{\text {reg, }} \omega\right)$ is not geodesically complete unless $X$ is smooth. The incompleteness of $\omega$ is a major obstacle to using the splitting theorems mentioned above or the Bochner principle directly in our setup. Consequently, it is highly challenging to analyse the geometry of $\left(X_{\text {reg }}, \omega\right)$ using differential-geometric techniques alone.

In this paper, we use recent advances in higher-dimensional algebraic geometry to study the geometry of the Kähler manifold $\left(X_{\mathrm{reg}}, \omega\right)$ and its relation to the global algebraic geometry of the projective variety $X$. More precisely, we will investigate the following.

- The holonomy group $G=\operatorname{Hol}\left(X_{\text {reg }}, g\right)$, where $g$ is the Riemannian metric on $X_{\text {reg }}$ induced by $\omega$.

- The algebra of global holomorphic forms $\mathcal{A}=\oplus_{p} H^{0}\left(X_{\mathrm{reg}}, \Omega_{X_{\mathrm{reg}}}^{p}\right)$.

- The fundamental group $\pi_{1}\left(X_{\text {reg }}\right)$.

Motivated by a decomposition theorem for tangent sheaves of klt varieties with numerically trivial canonical divisor established by Greb, Kebekus, and Peternell in [GKP16b] and building on Bost's criteria for algebraic integrability of foliations, Druel recently obtained the singular version of the Decomposition Theorem for such varieties of dimension $\operatorname{dim} X \leq 5$, see [Dru18]. Using Druel's strategy as well as the results presented in this paper, in particular Proposition D and Theorem E below, Höring and Peternell very recently gave a proof of the singular version of the Decomposition Theorem in [HP17], thus completing the long quest for such a result in the singular category.

1.1. The Bochner principle. There exist strong connections between the three objects listed above. First of all, it is in general significantly simpler to compute the 
neutral component $G^{\circ}$ of $G$, as $G^{\circ}$ is invariant under finite étale covers, and since all possible isomorphism classes were classified by Berger-Simons. The group of connected components, $G / G^{\circ}$, is then controlled by the fundamental group of the variety via the canonical surjection $\pi_{1}\left(X_{\text {reg }}\right) \rightarrow G / G^{\circ}$. Finally, the link between $G$ and $\mathcal{A}$ is provided in the smooth case by the Bochner principle, which is a straightforward application of the maximum principle to Bochner's formula. One of our main results consists in the following generalisation of the Bochner principle to the singular setting.

Theorem A (Bochner principle, Theorem 8.2). Let X be a projective klt variety with $K_{X}$ numerically trivial. Let $H$ be an ample divisor on $X$, and let $\omega_{H} \in c_{1}(H)$ be the singular Ricci-flat Kähler metric constructed by [EGZ09], with associated Riemannian metric $g_{H}$ on $X_{\text {reg. }}$. Then, every holomorphic tensor on $X_{\text {reg }}$ is parallel with respect to $g_{H}$.

The proof of this result is much more involved than in the smooth case. We first establish a Bochner principle for subbundles of tensor bundles, Theorem 8.1, using the analysis developed in [Gue16]. To obtain Theorem A, this is subsequently combined with group-theoretic arguments and with the existence of a certain "holonomy cover", which we explain in Theorem B below.

1.2. The holonomy cover. As we explained above, it is difficult in general to compute the full holonomy group, but rather easy to get our hands on its neutral component $G^{\circ}$, cf. Proposition 5.3. In the smooth setup, the passage from this component to the full holonomy group is facilitated by the a priori control over the fundamental group of Ricci-flat manifolds given by the Cheeger-Gromoll Theorem. In our setup, we have the following major potential problem: even if the (restricted) holonomy of the metric on $X_{\text {reg }}$ has no flat factors, the fundamental group $\pi_{1}\left(X_{\text {reg }}\right)$ might be infinite, and it is therefore not clear that we can make the holonomy group connected by taking a finite étale cover of $X_{\text {reg }}$.

However, we can overcome this potential topological obstruction by relying on recent progress in higher-dimensional algebraic geometry. Our two main technical ingredients for this part are Druel's integrability theorem, [Dru18, Thm. 1.4], and the theorem on the existence of maximally quasi-étale covers of klt varieties, [GKP16c, Thm. 1.5]. Combining these results with more elementary differentialgeometric considerations, we get the following.

Theorem B (Holonomy cover, Theorem 7.1 and Proposition 7.6). Setting as in Theorem $A$. Then, there exist normal projective varieties $A$ and $Z$, and a quasi-étale cover $\gamma: A \times Z \rightarrow X$ such that the following properties hold.

(1.1.1) The variety $A$ is Abelian, of dimension $\operatorname{dim} A=\widetilde{q}(X)$, the augmented irregularity of $X$.

(1.1.2) The variety $Z$ has canonical singularities, linearly trivial canonical divisor, and augmented irregularity $\widetilde{q}(Z)=0$.

(1.1.3) There exist a flat Kähler form $\omega_{A}$ on $A$ and a singular Ricci-flat Kähler metric $\omega_{Z}$ on $\mathrm{Z}$ such that $\gamma^{*} \omega_{H} \cong \operatorname{pr}_{1}^{*} \omega_{A}+\operatorname{pr}_{2}^{*} \omega_{Z}$ and such that the holonomy group of the corresponding Riemannian metric on $A \times Z_{\text {reg }}$ is connected.

Reminder 1.2. The augmented irregularity of $X$ is defined by Kawamata to be the maximal irregularity of any quasi-étale cover of $X$, see Section 2.7.

The singular Kähler-Einstein metric $\omega_{H}$ constructed by [EGZ09] does depend on the choice of the ample divisor $H$. However, using Theorems A and B to relate holonomy, restricted holonomy, and holomorphic differential forms, we will show that the isomorphism class of the restricted holonomy group $G^{\circ}$ is in fact independent of $H$, allowing us to speak of the restricted holonomy. The following proposition makes this precise. 
Proposition C (Restricted holonomy is independent of polarisation, Corollary 8.8). Setting as in Theorem A. Then, the isomorphism class of the restricted holonomy group $\mathrm{Hol}\left(X_{\mathrm{reg}}, g_{H}\right)^{\circ}$ does not depend on the ample polarisation $H$.

We will see in Section 3.2 that the construction of Ricci-flat Kähler metrics by [EGZ09] is well-behaved under quasi-étale cover, and then so is the restricted holonomy. In contrast, Section 14.1 shows by way of example that the restricted holonomy changes dramatically under birational modifications, even under crepant blowing up.

1.3. Decomposition of the tangent sheaf. Using the holonomy principle as well as the classification of Ricci-flat restricted holonomy groups, and comparing with the decomposition of the tangent sheaf of varieties with trivial canonical divisor established in [GKP16b], we can obtain more precise information about the tangent sheaf of $Z$, which splits according to the restricted holonomy representation of $X$.

Proposition D (Decomposition of the tangent sheaf, Proposition 7.9). Setup and notation as in Theorem B. Then, the cover $\gamma: A \times Z \rightarrow X$ can be chosen such that in addition to properties (1.1.1)-(1.1.3), there exists a direct sum decomposition of the tangent sheaf of $Z$,

$$
\mathscr{T}_{Z}=\bigoplus_{i \in I} \mathscr{E}_{i} \oplus \bigoplus_{j \in J} \mathscr{F}_{j}
$$

where the reflexive sheaves $\mathscr{E}_{i}$ (resp. $\mathscr{F}_{j}$ ) satisfy the following properties.

(1.2.1) The subsheaves $\mathscr{E}_{i} \subseteq \mathscr{T}_{Z}$ (resp. $\mathscr{F}_{j} \subseteq \mathscr{T}_{Z}$ ) are foliations with trivial determinant, of rank $n_{i} \geq 3$ (resp. of even rank $2 m_{j} \geq 2$ ). Moreover, they are strongly stable in the sense of [GKP16b, Def. 7.2].

(1.2.2) On $Z_{\text {reg, }}$ the $\mathscr{E}_{i}$ (resp. $\mathscr{F}_{j}$ ) are locally free and correspond to holomorphic subbundles $E_{i}$ (resp. $F_{j}$ ) of $T Z_{\mathrm{reg}}$ that are parallel with respect to the Levi-Civita connection of $g_{Z}$, the Riemannian metric on $Z_{\text {reg }}$ induced by $\omega_{Z}$. Moreover, their holonomy groups are $\mathrm{SU}\left(n_{i}\right)$ and $\mathrm{Sp}\left(m_{j}\right)$, respectively.

(1.2.3) If $x \in X_{\mathrm{reg}}$ and $(a, z) \in \gamma^{-1}(x)$, then the splitting

$$
T_{x} X \cong T_{(a, z)}(A \times Z)=T_{a} A \oplus \bigoplus_{i \in I} E_{i, z} \oplus \bigoplus_{j \in J} F_{j, z}
$$

corresponds to the decomposition of $T_{x} X$ into irreducible representations under the action of the restricted holonomy group $\operatorname{Hol}\left(X_{\mathrm{reg}}, g_{H}\right)$ at $x$.

Proposition D is a significant refinement of the decompositions obtained in [GKP16b] and [Gue16] and, as already mentioned above, is one of the ingredients in Höring-Peternell's proof for the singular analogue of the Decomposition Theorem in any dimension, see [HP17]. The irreducible pieces appearing in their result are the ones described in [GKP16b, Sect. 8.B]. We will discuss these in the next paragraph.

1.4. Irreducible pieces of the decomposition. Smooth Calabi-Yau manifolds and irreducible holomorphic symplectic manifolds are defined by two conditions: one is algebraic, expressed in terms of the algebra of holomorphic forms, and the other one is topological, namely simple connectedness. In particular, their holonomy group is connected and their algebra of holomorphic forms cannot be made any larger by taking finite étale covers. In the singular setting, [GKP16b, Def. 8.16] proposed the following purely algebro-geometric definition.

Definition 1.3 (CY and IHS). Let $X$ be a normal projective variety with $\mathscr{O}_{X} \cong \omega_{X}$ of dimension at least two, having at worst canonical singularities. 
(1.3.1) We call X Calabi-Yau (CY) if $H^{0}\left(Y, \Omega_{Y}^{[p]}\right)=\{0\}$ for all numbers $0<p<$ $\operatorname{dim} X$ and all finite, quasi-étale covers $Y \rightarrow X$.

(1.3.2) We call $X$ irreducible holomorphic symplectic (IHS) if there exists a holomorphic symplectic two-form $\sigma \in H^{0}\left(X, \Omega_{X}^{[2]}\right)$ such that for all finite, quasiétale covers $\gamma: Y \rightarrow X$, in particular for $X$ itself, the exterior algebra of global reflexive forms is generated by $\gamma^{[*]} \sigma$.

The combination of Theorem A and Theorem B above answers the natural question posed in [GKP16b] concerning the characterisation of these two classes of varieties in terms of the strong stability of their tangent sheaf.

Theorem E (Strongly stable varieties, Corollary 12.7). Let X be a projective klt variety of dimension at least two, with numerically trivial canonical divisor. Assume that $\mathscr{T}_{X}$ is strongly stable in the sense of [GKP16b, Def. 7.2]. Then, there exists a quasi-étale cover $\gamma: Y \rightarrow X$ such that $Y$ is Calabi-Yau or irreducible holomorphic symplectic.

In conclusion, a variety with klt singularities and numerically trivial canonical class admits a quasi-étale cover which is a CY or IHS variety if and only if its tangent bundle is strongly stable. Example 14.9 discusses a variety with canonical singularities, trivial canonical bundle and with no reflexive forms of intermediate degree that admits a quasi-étale cover which is an IHS variety.

In light of these results, one would like to propose an alternative definition of Calabi-Yau and irreducible holomorphic symplectic varieties that does not involve looking at quasi-étale covers. In other words, one would like to replace the assumption on simple connectedness. We want to emphasise that there does not seem to be an easy topological condition that would play its role in the singular setting.

- The assumption " $\pi_{1}(X)=\{1\}$ " is not the right one. Example 14.1 discusses a singular Kummer surface that is is simply connected and has the same algebra of reflexive forms as a smooth $K 3$. However, the example is a quotient of an Abelian variety.

- The assumption " $\pi_{1}\left(X_{\text {reg }}\right)=\{1\}$ " might seem like a good condition, but even in the IHS case, we do not know that $\pi_{1}\left(X_{\text {reg }}\right)$ is actually finite. Even worse, in the CY case $\pi_{1}\left(X_{\text {reg }}\right)$ could a priori be infinite with infinite completion for all we know.

However, due to the finiteness statement for $G / G^{\circ}$ contained in Theorem $\mathrm{B}$, a good condition to impose in place of simple connectedness is holonomy connectedness. This leads to the following characterisation, proven in Section 12.3.

Proposition F (Characterisation of CY and IHS by holonomy, Proposition 12.10). Setting as in Theorem A. Then, the following conditions are equivalent.

(1.3.3) $X$ is a Calabi-Yau variety.

(1.3.4) $\mathrm{Hol}\left(X_{\mathrm{reg}}, g_{H}\right)$ is connected and $H^{0}\left(X, \Omega_{X}^{[p]}\right)=\{0\}$ for all $0<p<n$.

(1.3.5) $\mathrm{Hol}\left(X_{\mathrm{reg}}, g_{H}\right)$ is isomorphic to $\mathrm{SU}(n)$.

Analogously, the following conditions are equivalent.

(1.3.6) $X$ is an irreducible holomorphic symplectic variety.

(1.3.7) $\mathrm{Hol}\left(X_{\mathrm{reg}}, g_{H}\right)$ is connected, and there exists a holomorphic symplectic two-form $\sigma \in H^{0}\left(X, \Omega_{X}^{[2]}\right)$ such that $\oplus_{p=0}^{n} H^{0}\left(X, \Omega_{X}^{[p]}\right)=\mathbb{C}[\sigma]$.

(1.3.8) $\mathrm{Hol}\left(X_{\mathrm{reg}}, g_{H}\right)$ is isomorphic to $\operatorname{Sp}\left(\frac{n}{2}\right)$.

We refer to Section 14 where lots of examples are given that compare our notions of $\mathrm{CY}$ and IHS varieties to some other ones existing in the literature and that emphasise the subtlety of the dichotomy question once singularities are allowed. 
1.5. Stability and irreducible (restricted) holonomy. Theorem B and the methods of its proof also establish correspondences between the algebro-geometric notion of stability and irreducibility of the differential-geometric holonomy representation.

Proposition G (Stability and irreducibility, Corollary 6.8 and 7.4). Setting as in Theorem A. Let $x \in X_{\mathrm{reg}}$, let $G$ (resp. $G^{\circ}$ ) be the holonomy group (resp. restricted holonomy group) of $\left(X_{\mathrm{reg}}, g_{H}\right)$ at $x$, and let $V:=T_{x} X$. Then, $\mathscr{T}_{X}$ is stable with respect to any ample polarisation (resp. strongly stable) if and only if the representation $G \circlearrowleft V$ (resp. $G^{\circ} \circlearrowleft V$ ) is irreducible.

The second result provides an algebro-geometric criterion for the vanishing of the augmented irregularity of a variety $X$ in terms of properties of holomorphic tensors on $X$ itself. This is achieved by considering invariants of the restricted holonomy representation.

Theorem $\mathbf{H}$ (Augmented regularity and symmetric differentials, Theorem 11.1). Setting as in Theorem $A$. Let $x \in X_{\text {reg, }}$, let $G^{\circ}$ be the restricted holonomy group of $\left(X_{\text {reg }}, g_{H}\right)$ at $x$, and let $V:=T_{x} X$. Then, the following are equivalent.

(1.3.9) We have $H^{0}\left(X, \operatorname{Sym}^{[m]} \Omega_{X}^{1}\right)=\{0\}$ for all $m \in \mathbb{N}^{+}$.

(1.3.10) The augmented irregularity of $X$ vanishes, $\widetilde{q}(X)=0$.

(1.3.11) The set of $G^{\circ}$-invariant vectors in $V$ is trivial, $V^{G^{\circ}}=\{0\}$.

1.6. Fundamental groups. In the smooth case, Theorem 1.1 shows that the fundamental group of $X$ is virtually Abelian, that is, an extension of $\mathbb{Z}^{r}$ by a finite group. In particular, if the augmented irregularity of $X$ vanishes, $\widetilde{q}(X)=0$, then $\pi_{1}(X)$ is actually finite. The proof of this fundamental result relies on the Cheeger-Gromoll theorem, which says that once an Euclidean space of maximal dimension is split off the universal cover of a complete Ricci-flat manifold $X$, the remaining factor is compact. As already mentioned above, the proof of the Cheeger-Gromoll theorem uses completeness $X$ in a fundamental way, so none of these methods apply to the non-complete Riemannian manifolds $\left(X_{\mathrm{reg}}, g_{H}\right)$ considered here.

Fortunately, the algebraicity of $X$ opens the door to alternative techniques, which allow us to prove the following finiteness result.

Theorem I (Finiteness of $\pi_{1}$, Theorem 13.1, 13.6, Corollary 13.2, 13.3, 13.10.). Let $X$ be a projective klt variety with numerically trivial canonical divisor.

(1.3.12) If $\mathscr{T}_{X}$ is strongly stable and if $\operatorname{dim} X$ is even, then $\pi_{1}(X)$ and $\hat{\pi}_{1}\left(X_{\mathrm{reg}}\right)$ are finite. If $X$ is IHS or an even-dimensional $C Y$, then $X$ is simply connected.

(1.3.13) If $\widetilde{q}(X)=0$, then $\pi_{1}\left(X_{\text {reg }}\right)$ does not admit any finite-dimensional representation with infinite image (over any field). Moreover, for each $n \in \mathbb{N}$, the fundamental group $\pi_{1}\left(X_{\mathrm{reg}}\right)$ admits only finitely many $n$-dimensional complex representations up to conjugation.

Remark 1.4. Under the assumption that $\widetilde{q}(X)=0$, the conclusions of Item (1.3.13) remain valid for $\pi_{1}(X)$ as well, given the surjection $\pi_{1}\left(X_{\text {reg }}\right) \rightarrow \pi_{1}(X)$ induced by the open immersion $X_{\text {reg }} \hookrightarrow X$, cf. [FL81, 0.7.B on p. 33].

About Item (1.3.12): the proof of finiteness of $\pi_{1}(X)$ strongly relies on the Bochner principle, and on methods introduced by Campana in [Cam95], which connect positivity properties of cotangent bundles to the size of fundamental groups. To

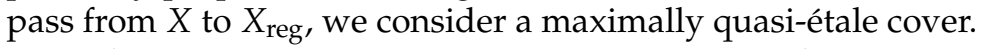

As for Item (1.3.13), the key point is a result of Brunebarbe-Klingler-Totaro, [BKT13], which links the existence of representations with infinite image to the 
existence of symmetric differentials. This, in turn, can be interpreted via the Bochner principle in terms of invariant vectors in the symmetric power of the standard representation of $\mathrm{SU}(n)$ or $\mathrm{Sp}(n)$, see Theorem $\mathrm{H}$ above.

1.7. Outline of the paper. The core of the paper consists in proving Theorems A and $B$ above. Interestingly enough, and unlike the strategy executed in the smooth case, the proof of Theorem A relies on the conclusions of Theorem B, which is proved in Part II. Part III is devoted to proving Theorem A using the results achieved in the earlier parts. More precisely, the content of the individual sections can be summarised as follows.

Part I. We recall the definitions and basic properties of the fundamental differential-geometric objects that will be used throughout the paper, namely the holonomy groups of a Kähler manifold and singular Kähler-Einstein metrics. We also analyse the behaviour of these objects with respect to quasi-étale covers.

Part II. This part is mostly taken up by the proof of Theorem 7.1. The starting point is the classification of restricted holonomy, Proposition 5.3, which shows that the usual dichotomy SU vs. Sp continues to hold in the singular setting.

Part III. We prove Theorem 8.1 stating that $\mathscr{T}_{X}$ or more generally any of its reflexive tensor powers is the direct orthogonal sum of stable parallel subbundles; the arguments follows [Gue16] closely. Capitalising on this and on the results of Part II, we establish the Bochner principle for forms and unfold a first list of applications in connection with augmented irregularity and characterisations of quotients of Abelian varieties, cf. Theorem 11.1 as well as Corollaries 8.5 and 11.4.

Part IV. We investigate the strongly stable case. Corollary 12.7 explains the relation to $\mathrm{CY}$ and IHS varieties in more detail. Section 12.4 relates our results to the algebraic holonomy group introduced by Balaji and Kollár. A number of examples illustrate the complexity of the situation. Finally, we study finiteness properties for the fundamental groups (resp. algebraic fundamental groups) of $X$ and $X_{\text {reg. }}$.

1.8. Acknowledgements. We would like to thank Stéphane Druel, Jochen Heinloth, Mihai Păun, Wolfgang Soergel, Matei Toma and Burt Totaro for helpful discussions, and user Holonomia on mathoverflow.net for answering our questions. We thank the anonymous referee for her/his time and efforts.

\section{Part I. Preparations}

\section{NOTATION AND CONVENTIONS}

The main motivation to study varieties with klt singularities comes from Minimal Model theory. On the other hand, many of our techniques originate in Differential Geometry. Hence, to make the paper more accessible for algebraic geometers, this chapter carefully sets up notation and gives very quick explanations of the terminology and of the fundamental principles used later.

2.1. Global conventions. Throughout the present paper, all varieties will be defined over the complex numbers. We will freely switch between the algebraic and analytic context if no confusion is likely to arise. If extra care is warranted, we denote the analytic space associated with an algebraic variety $X$ by $X^{a n}$. We follow the notation used in the standard reference books [Har77, KM98]. In particular, varieties are always assumed to be irreducible and reduced.

\subsection{Differential-geometric notions.}


2.2.1. Differentials and vector fields. Throughout this paper, we will clearly distinguish between bundles and their associated sheaves of smooth (resp. holomorphic) sections.

Notation 2.1 (Tangent bundle). Given a connected complex manifold $X$ of complex dimension $n$, denote the holomorphic tangent bundle by $T X$, the holomorphic tangent sheaf by $\mathscr{T}_{X}$, and the sheaf of $\mathcal{C}^{\infty}$-sections of TX as $\mathcal{T}_{X}$. If $x \in X$ is any point, let $T_{x} X$ be the associated tangent space, $\operatorname{dim}_{\mathbb{C}} T_{X} X=n$. The complexified tangent bundle decomposes as $T X^{\mathrm{C}}=T^{1,0} X \oplus T^{0,1} X$, where $T X$ and $T^{1,0} X$ are naturally isomorphic as complex vector bundles.

Notation 2.2 ( $\mathcal{C}^{\infty}$-functions and forms). Given a connected complex manifold $X$, let $\mathcal{A}_{X}$ denote the sheaf of complex-valued $\mathcal{C}^{\infty}$-functions on $X$. The symbols $\mathcal{A}_{X}^{p}$ denote the sheaves of complex, $\mathcal{C}^{\infty}$-differential $p$-forms. Likewise, $\mathcal{A}_{X}^{p, q}$ are the sheaves of complex, $\mathcal{C}^{\infty}$-differential of type $(p, q)$. The sheaves of holomorphic differentials are denoted by $\Omega_{X}^{p}$. There are a few standard variants of the notation that we will also use. If $E$ is a complex vector bundle, we denote the vector space of global, smooth, $E$-valued $(p, q)$-forms by $\mathcal{A}^{p, q}(X, E)$. If $E$ is equipped with a Hermitian metric $h$, we write $\mathcal{A}^{p, q}(X, \operatorname{End}(E, h))$ for the space of forms with values in Hermitian endomorphisms. Vector spaces of real forms will be denoted as $\mathcal{A}_{\mathbb{R}}^{p, q}(\bullet)$.

Notation 2.3 (Chern connections). Let $(X, \omega)$ be a connected Kähler manifold of dimension $n:=\operatorname{dim}_{C} X$. Let $h$ be the associated Hermitian metric on $T^{1,0} X$ and $g$ the associated Riemannian metric on $X$, cf. [Huy05, App. 4.A]. Owing to the Kähler property, the Levi-Civita connection of $g$ coincides with the Chern connection of $h$ after identifying $T X$ and $T^{1,0} X$, see [Huy05, Prop. 4.A.9]. We obtain induced complex connections on all tensor bundles $T X^{\otimes p} \otimes T^{*} X^{\otimes q}$, which by minor abuse of notation we all denote by $D$.

2.2.2. Holonomy. The holonomy group of a Riemannian manifold is the core notion of this paper.

Notation 2.4. Let $(M, g)$ be a connected Riemannian manifold. Given a point $m \in$ $M$, we view $\left(T_{m} M, g_{m}\right)$ as a Euclidean vector space and denote the associated Riemannian holonomy group by $\operatorname{Hol}(M, g)_{m}$, which we view as a subgroup of the orthogonal group $\mathrm{O}\left(T_{m} M, g_{m}\right)$. Its identity component, the restricted holonomy group, will be denoted by $\operatorname{Hol}(M, g)_{m}^{\circ}$.

The following relation between the holonomy group and the topology of the underlying manifold is crucial for our arguments.

Reminder 2.5 (Holonomy and fundamental group, [Joy00, Prop. 2.3.4]). Let $(M, g)$ be a connected Riemannian manifold. Then, for any point $m \in M$, as the restricted holonomy group coincides with those elements in $\operatorname{Hol}(M, g)_{m}$ that arise via parallel transport along contractible paths, we have a surjective group homomorphism

$$
\pi_{1}(M, m) \rightarrow \operatorname{Hol}(M, g)_{m} / \operatorname{Hol}(M, g)_{m}^{\circ} .
$$

Reminder 2.6 (Holonomy of Kähler manifolds, [Bes87, Sect. 10.C]). Let $(X, \omega)$ be a connected Kähler manifold. Let $h$ be the associated Hermitian metric on TX. Given any point $x \in X$, view $T_{x} X$ as a complex, Hermitian vector space. Since the complex structure on $X$ is parallel, the Riemannian holonomy group $\operatorname{Hol}(X, g)_{x}$ is contained in the unitary group $\mathrm{U}\left(T_{x} X\right)$. The Kähler manifold $(X, \omega)$ is Ricci-flat if and only if the restricted holonomy group $\operatorname{Hol}(X, g)_{x}^{\circ}$ is contained in $\mathrm{SU}\left(T_{x} X\right)$. 
Reminder 2.7 (The holonomy principle for complex tensors, [Bes87, Fundamental Principle 10.19]). Let $(X, \omega)$ be a connected Kähler manifold with associated Riemannian metric $g$. Choose $x \in X$ and consider the holonomy group $\operatorname{Hol}(X, g)_{x}$. The representation of $\operatorname{Hol}(X, g)_{x}$ on $T_{x} X$ naturally can be complexified to a representation on $T_{x}^{\mathrm{C}} X$, and as the original representation commutes with the complex structure $J_{x} \in \operatorname{End}\left(T_{x} X\right)$, it also induces representations on $T_{x}^{1,0} X$ and $T_{x}^{0,1} X$. The aforementioned isomorphism of $T X$ and $T^{1,0} X$ yields an isomorphism of representations $T_{x} X \cong T_{x}^{1,0} X$, and, as the representation of $\operatorname{Hol}(X, g)_{x}$ on $T_{x} X$ is unitary with respect to $h_{x}$, we hence obtain equivariant isomorphisms $T_{x}^{*} X \cong \overline{T_{x}^{1,0} X}=$ $T_{x}^{0,1} X$. By standard linear algebra, we obtain induced representations on the tensor spaces $T_{x} X^{\otimes p} \otimes_{\mathcal{C}} T_{x}^{*} X^{\otimes q}$. On the other hand, we have seen in Notation 2.3 that the Levi-Civita- resp. Chern-connection on $T X$ resp. $T^{1,0} X$, cf. Notation 2.3, induces connections $D$ on all the complex tensor bundles $T X^{\otimes p} \otimes T^{*} X^{\otimes q}$.

The holonomy principle states that evaluation at $x$ and parallel transport establish a C-linear one-to-one correspondence between tensors $t \in H^{0}\left(X, \mathcal{T}_{X}^{\otimes p} \otimes_{\mathcal{A}_{X}}\right.$ $\left(\mathcal{T}_{X}^{*}\right)^{\otimes q}$ ) with vanishing covariant derivative, $D t=0$, which are automatically holomorphic as the $(0,1)$-part of $D$ coincides with the complex structure $\bar{\partial}$, and invariant vectors $t_{x}$ in the corresponding unitary $\operatorname{Hol}(X, g)_{x}$-representation $T_{x} X^{\otimes p} \otimes_{C} T_{x}^{*} X^{\otimes q}$. For more on the compatibility of parallel transport and the holomorphic structure, we refer to Section 4.5 below.

The symplectic group appears prominently in the classification of holonomy groups. We use the following convention.

Notation 2.8 (Symplectic groups). Let $n \in \mathbb{N}^{+}$and let $\sigma:=\sum_{k=1}^{n} d z_{k} \wedge d z_{n+k}$ be the standard complex symplectic form on $\mathbb{C}^{2 n}$. We denote by $\operatorname{Sp}(n, \mathbb{C})$ the complex symplectic group, that is, the subgroup of $\mathrm{GL}(2 n, \mathbb{C})$ consisting of transformations preserving $\sigma$. We denote by $\operatorname{Sp}(n)$ its compact real form, $\operatorname{Sp}(n):=\operatorname{Sp}(n, \mathbb{C}) \cap$ $\mathrm{U}(2 n)$, the unitary symplectic group.

2.3. Local decomposition of Kähler manifolds. The relation between holonomy groups and stability properties of the tangent sheaves will be established by using the following folklore result, which we include here for lack of a reference.

Proposition 2.9 (Local decomposition of Kähler manifolds). Let $(X, \omega)$ be a simply connected Kähler manifold (not necessarily compact or complete) and $x \in X$ a point. Then there exists an open neighbourhood $U=U(x)$, Kähler manifolds $\left(U_{i}, \omega_{i}\right)_{i=0 \ldots m}$ and an isomorphism of Kähler manifolds

$$
\varphi:(U, \omega) \rightarrow\left(\underset{i=0}{\stackrel{m}{\times}} U_{i}, \sum_{i=0}^{m} \pi_{i}^{*} \omega_{i}\right)
$$

such that the following holds when we write $\left(x_{0}, \ldots, x_{m}\right)$ for $\varphi(x)$ and $g, g_{i}$ for the associated Riemannian metrics on $X$ and on the $U_{i}$, respectively.

(2.9.2) The action of the holonomy group $H:=\operatorname{Hol}(X, g)_{x}$ on $T_{x} X$ respects the orthogonal decomposition $T_{x} X=\bigoplus_{i=0}^{m} T_{x_{i}} U_{i}$ induced by $\varphi$.

(2.9.3) The holonomy group is a direct product, $H=\times_{i=1}^{m} H_{i}$, where each factor $H_{i}$ acts irreducibly on the summand $T_{x_{i}} U_{i}$ and trivially on all the other summands.

Remark 2.10. The summand $T_{x_{0}} U_{0}$ is precisely the set of $H$-fixed vectors.

Proof of Proposition 2.9. Apply [KN96a, Thm. 5.4 on p. 185] to the Riemannian manifold $(X, g)$, in order to find a neighbourhood $U=U(x) \subseteq X$ that admits an isometry to a Riemannian product, $\varphi: U \rightarrow \times U_{i}$, satisfying (2.9.2) and (2.9.3). The proof of [KN96b, Thm. 8.1 on p. 172] applies nearly verbatim to show that the $U_{i}$ 
carry natural complex structures with respect to which the $g_{i}$ are Kähler and that make the isometry $\varphi$ biholomorphic.

Definition 2.11 (Totally decomposed actions). If $V$ is a Hermitian vector space and $G \subseteq U(V)$ any group, we call the action $G \circlearrowleft V$ totally decomposed if there exists a G-invariant orthogonal decomposition $V=V_{0} \oplus V_{1} \oplus \cdots \oplus V_{m}$, and a product decomposition, $G=G_{1} \times \cdots \times G_{m}$, where each factor $G_{i}$ acts non-trivially and irreducibly on $V_{i}$ and trivially on all the other summands.

Remark 2.12 (Uniqueness of decompositions). If $V$ is a unitary vector space and $G \subseteq U(V)$ any group, there are usually many $G$-invariant orthogonal decompositions of $V$ into complex vector spaces, $V=V_{0} \oplus V_{1} \oplus \cdots \oplus V_{m}$, where $G$ acts trivially on $V_{0}$ and non-trivially and irreducibly on the remaining summands. If the G-action is totally decomposed, then the decomposition is unique up to permutation of the $\left(V_{i}\right)_{i>0}$. For a proof, observe that the product structure of $G$ implies that a vector $\vec{v} \in V$ is contained in one of the $V_{i}$ if and only if the linear span of its $G$-orbit is an irreducible representation space (and then equal to $V_{i}$ ). The product structure of $G$ is also unique, as $G_{i}=\cap_{j \neq i} \operatorname{Fix}\left(V_{j}\right)$.

2.4. Varieties and sets. Normal varieties are $S_{2}$, which implies that regular functions can be extended across sets of codimension two. The following notation will be used.

Notation 2.13 (Big and small subsets). Let $X$ be a normal, quasi-projective variety. A Zariski-closed subset $Z \subset X$ is called small if $\operatorname{codim}_{X} Z \geq 2$. A Zariski-open subset $U \subseteq X$ is called big if $X \backslash U$ is small.

2.5. Morphisms. Galois morphisms appear prominently in the literature, but their precise definition is not consistent. We will use the following definition, which does not ask Galois morphisms to be étale.

Definition 2.14 (Covers and covering maps, Galois morphisms). A cover or covering map is a finite, surjective morphism $\gamma: Y \rightarrow X$ of normal, quasi-projective varieties. The covering map $\gamma$ is called Galois if there exists a finite group $G \subseteq \operatorname{Aut}(Y)$ such that $X$ is isomorphic to the quotient map $Y \rightarrow Y / G$.

Definition 2.15 (Quasi-étale morphisms). A morphism $\gamma: Y \rightarrow X$ between normal varieties is called quasi-étale if $\gamma$ is of relative dimension zero and étale in codimension one. In other words, $\gamma$ is quasi-étale if $\operatorname{dim} Y=\operatorname{dim} X$ and if there exists a closed, subset $Z \subseteq Y$ of codimension $\operatorname{codim}_{Y} Z \geq 2$ such that $\left.\gamma\right|_{Y \backslash Z}: Y \backslash Z \rightarrow X$ is étale.

Reminder 2.16 (KLT is invariant under quasi-étale covers). Let $\gamma: Y \rightarrow X$ be a quasi-étale cover. By definition, $\gamma$ is then quasi-étale, finite and surjective. If $K_{X}$ is Q-Cartier, then $K_{Y}=\gamma^{*} K_{X}$ is $\mathbb{Q}$-Cartier as well. If there exists a $\mathbb{Q}$-divisor $D$ on $X$ that makes $(X, D)$ is klt, then $\left(Y, \gamma^{*} D\right)$ is klt as well, [KM98, Prop. 5.20].

Remark 2.17 (Quasi-étale covers vs. étale covers of $X_{\text {reg }}$ ). If $\gamma: Y \rightarrow X$ is any quasiétale cover, purity of the branch locus implies that $\gamma$ is étale over $X_{\text {reg. Conversely, }}$. if $\gamma^{\circ}: Y^{\circ} \rightarrow X_{\text {reg }}^{a n}$ is a finite and locally biholomorphic morphism of complex manifolds, recall from [Gro71, Sect. XII.5] that $\gamma^{\circ}$ and $Y^{\circ}$ are algebraic, and can be compactified to a quasi-étale cover $\gamma: Y \rightarrow X$, see also [DG94, Thm. 3.4]. Consequently, we obtain obvious equivalences between the categories of quasiétale covers of $X$, of finite and étale covers of $X_{\text {reg }}$, and of finite sets with transitive action of $\pi_{1}\left(X_{\text {reg }}^{a n}\right)$.

Quasi-étale morphisms appear naturally in the context considered in this paper, as exemplified by the following important result. 
Proposition 2.18 (Global index one cover). Let $X$ be a projective, klt variety with numerically trivial canonical divisor, $K_{X} \equiv 0$. Then, there exists a quasi-étale cover $\gamma: Y \rightarrow X$ such that $Y$ has canonical singularities and linearly trivial canonical divisor, $K_{Y} \sim 0$.

Proof. By Nakayama's partial solution of the Abundance Conjecture, [Nak04, Cor. 4.9], there exists a number $m \in \mathbb{N}^{+}$such that $m \cdot K_{Y}$ is linearly equivalent to zero. Let $\gamma: Y \rightarrow X$ be the associated global index-one cover, [KM98, Def. 5.19], which is quasi-étale. By Reminder 2.16, the variety $Y$ is then klt. Moreover, by construction $K_{Y} \sim 0$, and the singularities of $Y$ are hence canonical.

2.6. Sheaves. Reflexive sheaves are in many ways easier to handle than arbitrary coherent sheaves, and we will therefore frequently take reflexive hulls. The following notation will be used.

Notation 2.19 (Reflexive hull). Given a normal, quasi-projective variety $X$ and a coherent sheaf $\mathscr{E}$ on $X$ of rank $r$, write

$$
\Omega_{X}^{[p]}:=\left(\Omega_{X}^{p}\right)^{* *}, \quad \mathscr{E}^{[m]}:=\left(\mathscr{E}^{\otimes m}\right)^{* *}, \quad \operatorname{Sym}^{[m]} \mathscr{E}:=\left(\operatorname{Sym}^{m} \mathscr{E}\right)^{* *}
$$

and $\operatorname{det} \mathscr{E}:=\left(\wedge^{r} \mathscr{E}\right)^{* *}$. Given any morphism $f: Y \rightarrow X$, write $f^{[*]} \mathscr{E}:=\left(f^{*} \mathscr{E}\right)^{* *}$.

2.7. Augmented irregularity. The irregularity of normal, projective varieties is generally not invariant under quasi-étale maps, even if the varieties in question are klt. The notion of "augmented irregularity" addresses this issue.

Definition 2.20 (Augmented irregularity). Let $X$ be a normal, projective variety. We denote the irregularity of $X$ by $q(X):=h^{1}\left(X, \mathscr{O}_{X}\right)$ and define the augmented irregularity as

$$
\widetilde{q}(X):=\sup \{q(Y) \mid Y \text { a quasi-étale cover of } X\} \in \mathbb{N} \cup\{\infty\} .
$$

Lemma 2.21 (Augmented irregularity and quasi-étale covers). Let $X$ be a normal, projective variety. Then, the following holds.

(2.21.1) The augmented irregularity is invariant under quasi-étale covers. More precisely, if $Y \rightarrow X$ is quasi-étale, then $\widetilde{q}(Y)=\widetilde{q}(X)$.

(2.21.2) If $X$ is a projective, klt variety with numerically trivial canonical class, then $\widetilde{q}(X) \leq \operatorname{dim} X$. In particular, the augmented irregularity is finite in this case.

(2.21.3) If $\widetilde{X} \rightarrow X$ is a birational morphism of projective varieties with canonical singularities, and if $K_{\widetilde{X}}$ is numerically trivial, then $\widetilde{q}(\widetilde{X}) \leq \widetilde{q}(X)$.

Proof. To prove Item (2.21.1), recall from [Laz04, Lem. 4.1.14] ("Injectivity Lemma") that the irregularity increases in covers and observe that any two quasiétale covers are dominated by a common third. Item (2.21.3) is shown in [Dru18, Lem. 4.4]. To prove Item (2.21.2), let $\gamma: Y \rightarrow X$ be a global index one cover, whose existence is guaranteed by Proposition 2.18. We may then apply [GKP16b, Rem. 3.4] and Item (2.21.1) to conclude.

\section{SINGULAR KÄHLER-EINSTEIN METRICS}

In this section, we recall the construction of Eyssidieux, Guedj and Zeriahi [EGZ09] $^{1}$, which produces so-called singular Kähler-Einstein metrics on certain singular varieties such as projective klt varieties with torsion canonical bundle. These objects induce genuine Kähler-Einstein metrics on the regular locus of the variety, and we will study the resulting holonomy groups.

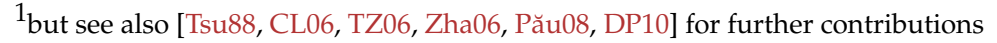


3.1. Existence of Ricci-flat Kähler metrics. Let $X$ be a projective, klt variety whose canonical class $K_{X}$ is numerically trivial. In this setting, Eyssidieux, Guedj and Zeriahi have shown that each Kähler class $\alpha \in H^{2}(X, \mathbb{R})$ contains a unique Ricci-flat Kähler metric $\omega_{\alpha}$ that is smooth on $X_{\text {reg }}$, satisfies Ric $\omega_{\alpha}=0$ there, and has bounded potentials near the singularities. To give a precise account of their construction, we need to specify notation first. We refer to reader to [EGZ09, Sect. 7.1] and [BEG13, Sect. 4.6.2] for proofs and further details.

Notation 3.1. Let $X$ be a projective, klt variety whose canonical class $K_{X}$ is numerically trivial. By Proposition 2.18 there exists a positive number $m \in \mathbb{N}^{+}$ such that $m \cdot K_{X}$ is linearly trivial. Choose one such $m$, choose a global generator $\sigma \in H^{0}\left(X, \mathscr{O}_{X}\left(m \cdot K_{X}\right)\right)$, choose a constant Hermitian metric $\|\cdot\|$ on the trivial bundle $\mathscr{O}_{X}\left(m \cdot K_{X}\right)$ and consider the following volume form on $X_{\text {reg, }}$

$$
i^{n} \cdot(-1)^{\frac{n(n+1)}{2}} \cdot\left(\frac{\sigma \wedge \bar{\sigma}}{\|\sigma\|^{2}}\right)^{\frac{1}{m}} \text { where } n:=\operatorname{dim} X .
$$

Finally, let $\mu_{X}$ be the associated positive measure on $X$ obtained as the trivial extension of the measure on $X_{\text {reg }}$ associated with the volume form in (3.1.1) above.

Remark 3.2 (Independence on choices). The volume form in (3.1.1) and the measure $\mu_{X}$ are easily seen to be independent of the choice of $m$ and $\sigma$. The measure $\mu_{X}(X)^{-1} \cdot \mu_{X}$ is independent of the number $m$, the form $\sigma$ and of the choice of the constant Hermitian metric $\|\cdot\|$.

Theorem 3.3 (Existence of Ricci-flat Kähler metrics, cf. [EGZ09, Thm. 7.5]). Let X be a projective, klt variety whose canonical class $K_{X}$ is numerically trivial. Given any ample $H \in \operatorname{Div}(X)$ with class $[H] \in H^{2}(X, \mathbb{R})$, there exists a unique closed positive current $\omega_{H}$ on $X$ such that the following holds.

(3.3.1) Denoting the de Rham cohomology class of the current $\omega_{H}$ by $\left[\omega_{H}\right]$, we have an equality of cohomology classes, $\left[\omega_{H}\right]=[H] \in H^{2}(X, \mathbb{R})$.

(3.3.2) The current $\omega_{H}$ has bounded potentials.

(3.3.3) Using Notation 3.1, the positive measure $\left(\omega_{H}\right)^{\operatorname{dim} X}$ obtained as the top intersection of $\omega_{H}$ puts no mass on proper analytic subsets and satisfies

$$
\left(\omega_{H}\right)^{\operatorname{dim} X}=[H]^{\operatorname{dim} X} \cdot \mu_{X}(X)^{-1} \cdot \mu_{X} .
$$

Furthermore, the current $\omega_{H}$ is smooth on $X_{\text {reg }}$ and induces a genuine Ricci-flat Kähler metric there.

Explanation (Item (3.3.2), bounded potentials). Choose a smooth reference Kähler metric $\omega$ on $X$ with $[\omega]=[H]$ and say that " $\omega_{H}$ has bounded potentials" if one can write $\omega_{H}=\omega+d d^{c} \varphi$ for some bounded function $\varphi$. This condition is independent of the choice of the Kähler metric $\omega \in[H]$, as any two cohomologous Kähler metrics differ by the $d d^{c}$ of a smooth function, cf. [BEG13, Sect. 4.6.1, discussion below Def. 4.6.2].

Explanation (Item (3.3.3), top intersection of currents and Ricci curvature). Bedford-Taylor theory allows to define the top intersection $\left(\omega_{H}\right)^{\operatorname{dim} X}$ of the current $\omega_{H}$, which is a positive measure on $X$ that puts no mass on analytic, or more generally pluripolar sets. Indeed, if $E$ is pluripolar, write $\omega_{H}=d d^{c} \varphi$ for some local psh function $\varphi$, take a locally defined psh function $V$ with $\{V=-\infty\}=E$ and apply [Dem85, Thm. 2.2(b)] with $k=n$, and with $\varphi_{1}:=\varphi, \ldots, \varphi_{n}:=\varphi$. The equation in Item (3.3.3) expresses that Ric $\omega_{H}=0$ in the sense of currents.

Remark 3.4 (KLT versus canonical singularities in Theorem 3.3). The statement [EGZ09, Thm. 7.5] assumes that $X$ has canonical singularities. The proof does 
not use this assumption and works verbatim for klt spaces in case where $\Delta=0$, compare with [EGZ09, Thm. 7.12].

3.2. Universal property of the EGZ construction. The construction of Theorem 3.3 has the following universal property.

Proposition 3.5 (Universal property of the EGZ construction). Let X be projective, klt variety whose canonical class $K_{X}$ is numerically trivial. Let $H \in \operatorname{Div}(X)$ be ample. Given a quasi-étale cover $\gamma: Y \rightarrow X$, then $H_{Y}:=\gamma^{*} H$ is ample. Recalling from Reminder 2.16 that $Y$ is klt and that $K_{Y}=\gamma^{*} K_{X}$ is numerically trivial, Theorem 3.3 applies both to $(X, H)$ and to $\left(Y, H_{Y}\right)$, and defines genuine Ricci-flat Kähler metrics $\omega_{H}$ and $\omega_{H_{Y}}$ on $X_{\text {reg }}$ and $Y_{\text {reg, }}$, respectively. These metrics agree on the smooth, open set $Y^{\circ}:=\gamma^{-1}\left(X_{\text {reg }}\right)$. In other words, $\omega_{H_{Y}} \mid Y^{\circ}=\left(\gamma \mid Y^{\circ}\right)^{*} \omega_{H}$.

Remark (Pull-back of currents). Recall that unlike arbitrary currents, closed positive $(1,1)$-currents can be pulled back under holomorphic maps. More precisely, if $f: Y \rightarrow X$ is a holomorphic map between two normal compact Kähler spaces and if $T$ is a closed positive $(1,1)$-current on $X$, then one can write $T=\omega+d d^{c} \varphi$ for some smooth form $\omega$ and some $\omega$-psh function $\varphi$. The closed positive $(1,1)$ current $f^{*} T$ on $Y$ is defined to be $f^{*} T:=f^{*} \omega+d d^{c}(\varphi \circ f)$. This pull-back is easily seen to extend the usual pull-back of forms.

Proof of Proposition 3.5. Choose a global generator $\sigma \in H^{0}\left(X, \mathscr{O}_{X}\left(m \cdot K_{X}\right)\right)$ and a constant Hermitian metric $\|\cdot\|$ on the trivial bundle $\mathscr{O}_{X}\left(m \cdot K_{X}\right)$. We obtain a positive measure $\mu_{X}$ on $X$, as in Notation 3.1. Consider the pull-back form $\gamma^{*} \sigma$, which generates $\mathscr{O}_{Y}\left(m \cdot K_{Y}\right)$, as well as the constant pull-back metric $\|\cdot\|$ on this trivial bundle. We obtain the associated measure $\mu_{Y}$ on $Y$. Write $n:=\operatorname{dim} X$ and consider the positive numbers

$$
C:=[H]^{n} \cdot \mu_{X}(X)^{-1}>0 \quad \text { and } \quad C_{Y}:=\left[H_{Y}\right]^{n} \cdot \mu_{Y}(Y)^{-1}>0 .
$$

We claim that $\gamma^{*} \omega_{H}=\omega_{H_{\gamma}}$, from which Proposition 3.5 would follow. For this, it suffices check that the pull-back current $\gamma^{*} \omega_{H}$ satisfies Properties (3.3.1)-(3.3.3) that uniquely characterise $\omega_{H_{Y}}$. Since $\gamma^{*} \omega_{H}$ clearly lives in $\left[H_{Y}\right]$ and has bounded potentials, it only remains to show that its top intersection $\left(\gamma^{*} \omega_{H}\right)^{n}$ equals $\widetilde{C} \cdot \mu_{\gamma}$. Pulling back the relation $\omega_{H}^{n}=C \cdot \mu_{X}$ by $\gamma$, we obtain an equality of measures on $\gamma^{\circ}=\gamma^{-1}\left(X_{\text {reg }}\right)$,

$$
\left(\gamma^{*} \omega_{H}\right)^{n}=C \cdot i^{n}(-1)^{\frac{n(n+1)}{2}}\left(\frac{\gamma^{*} \sigma \wedge \overline{\gamma^{*} \sigma}}{\left\|\gamma^{*} \sigma\right\|^{2}}\right)^{\frac{1}{m}}
$$

Over $Y^{\circ}$, where $\gamma$ is étale, the section $\gamma^{*} \sigma$ trivialises $\mathscr{O}_{Y}\left(\gamma^{*}\left(m \cdot K_{X}\right)\right)=\mathscr{O}_{Y}\left(m \cdot K_{Y}\right)$. We hence conclude from Remark 3.2 that the following measures coincide on $Y^{\circ}$,

$$
\mu_{Y}=i^{n}(-1)^{\frac{n(n+1)}{2}}\left(\frac{\gamma^{*} \sigma \wedge \overline{\gamma^{*} \sigma}}{\left\|\gamma^{*} \sigma\right\|^{2}}\right)^{\frac{1}{m}} \quad \text { on } Y^{\circ} .
$$

Combining (3.5.1) and (3.5.2), we get that

$$
\left(\gamma^{*} \omega_{H}\right)^{n}=C \cdot \mu_{\Upsilon} \quad \text { on } \gamma^{\circ} .
$$

But none of the two measures in (3.5.3) charges pluripolar sets: for the LHS, this is because $\gamma^{*} \omega_{H}$ has bounded potentials; for the RHS this is almost by definition. On the other hand, $Y \backslash Y^{\circ}$ is a proper analytic subset of $Y$, hence pluripolar. We conclude that the two measures coincide, and that the equality $\left(\gamma^{*} \omega_{H}\right)^{n}=C \cdot \mu_{Y}$ holds globally on $Y$. Finally, observe that

$$
\mu_{Y}(Y)=\mu_{Y}\left(Y^{\circ}\right)=(\operatorname{deg} \gamma) \cdot \mu_{X}\left(X_{\mathrm{reg}}\right)=\frac{(\operatorname{deg} \gamma) \cdot[H]^{n}}{C}=\frac{\left[H_{Y}\right]^{n}}{C} .
$$


This proves the desired equality $C=\left[H_{Y}\right]^{n} \cdot \mu_{Y}(Y)^{-1}=C_{Y}$.

Remark 3.6. It follows from the above result that the current $\gamma^{*} \omega_{H}$ is smooth and Kähler on the open set $Y_{\text {reg }} \supseteq \gamma^{-1}\left(X_{\text {reg }}\right)$ although $\gamma$ may not be étale there. For a typical example, consider a situation where $X$ has quotient singularities and admits a global, smooth, quasi-étale cover $\gamma: Y \rightarrow X$.

3.3. Product situations. Another straightforward though useful property of the EGZ construction is its compatibility with product structures. In the smooth case, this follows from the fact that the Ricci curvature of a product metric is the product of the Ricci forms, and that cohomology classes also behave naturally. In the singular case, one has to argue at the level of the Monge-Ampère equations satisfied by the Kähler-Einstein metrics.

Proposition 3.7 (EGZ construction in product situation). Let $X_{1}$ and $X_{2}$ be projective, klt varieties whose canonical classes $K_{X}$ are numerically trivial. Let $H_{\bullet} \in \operatorname{Div}\left(X_{\bullet}\right)$ be ample. Then, $X=X_{1} \times X_{2}$ is klt, with trivial canonical class and $H:=\left(\mathrm{pr}_{1}\right)^{*} H_{1} \times$ $\left(\mathrm{pr}_{2}\right)^{*} \mathrm{H}_{2} \in \operatorname{Div}(X)$ is ample. Theorem 3.3 applies to $\left(X_{\bullet}, H_{\bullet}\right)$ and to $(X, H)$, and defines genuine Ricci-flat Kähler metrics $\left(\omega_{H_{\bullet}}\right)$ and $\omega_{H}$ on $\left(X_{\bullet}\right)_{\text {reg }}$ and on $X_{\text {reg, respect- }}$ ively. With this notation, the Ricci-flat Kähler manifold $\left(X_{\mathrm{reg}}, \omega_{H}\right)$ is isomorphic to the product $\left(\left(X_{1}\right)_{\mathrm{reg}}, \omega_{H_{1}}\right) \times\left(\left(X_{2}\right)_{\mathrm{reg}}, \omega_{\mathrm{H}_{2}}\right)$.

Proof. The current $\omega:=\operatorname{pr}_{1}^{*} \omega_{H_{1}}+\mathrm{pr}_{2}^{*} \omega_{H_{2}}$ on $X$ has bounded potentials and satisfies $[\omega]=[H]$. In order to prove the identity of current $\omega=\omega_{H}$ on $X$ from which the proposition follows, it will therefore suffice to check Property (3.3.3) of Theorem 3.3. To this end, observe that if $m \in \mathbb{N}$ is divisible enough, one has a natural isomorphism

$$
H^{0}\left(X, \mathscr{O}_{X}\left(m \cdot K_{X}\right)\right) \cong H^{0}\left(X_{1}, \mathscr{O}_{X_{1}}\left(m \cdot K_{X_{1}}\right)\right) \otimes H^{0}\left(X_{2}, \mathscr{O}_{X_{2}}\left(m \cdot K_{X_{2}}\right)\right) .
$$

It follows that the product measure $\mu_{X_{1}} \otimes \mu_{X_{2}}$ coincides with $\mu_{X}$ up to a constant. In particular,

and therefore

$$
\frac{\mu_{X}}{\mu_{X}(X)}=\frac{\mu_{X_{1}} \otimes \mu_{X_{2}}}{\mu_{X_{1}}\left(X_{1}\right) \cdot \mu_{X_{2}}\left(X_{2}\right)}
$$

$$
\frac{[H]^{\operatorname{dim} X} \cdot \mu_{X}}{\mu_{X}(X)}=\frac{\left[H_{1}\right]^{\operatorname{dim} X_{1}} \cdot\left[H_{2}\right]^{\operatorname{dim} X_{2}} \cdot \mu_{X_{1}} \otimes \mu_{X_{2}}}{\mu_{X_{1}}\left(X_{1}\right) \cdot \mu_{X_{2}}\left(X_{2}\right)} .
$$

The product current $\omega$ therefore satisfies Property (3.3.3), as desired.

\section{THE STANDARD SETTING}

The goal of this section is to set up the framework for the rest of the article. In addition, we recollect some results about holonomy that we will use repeatedly later on. This includes locality of restricted holonomy, relation with parallel transport and behaviour under quasi-étale covers.

4.1. The standard setting. Throughout the present paper we will be working in the setup of Theorem 3.3, and use the metrics produced there in order to compute holonomy groups. We will use the following notation.

Setup and Notation 4.1 (Standard setting). Let $X$ be a projective, klt variety with numerically trivial canonical class $K_{X}$. Write $n:=\operatorname{dim}_{C} X$ and assume that $n \geq 2$. Fix an ample Cartier divisor $H$ on $X$ and a smooth point $x \in X_{\text {reg. }}$. Let $\omega_{H}$ denote the singular, Ricci-flat Kähler metric constructed in Theorem 3.3. Write $g_{H}$ for the associated Riemannian metric on $X_{\text {reg, }}$ and $h_{H}$ for the associated Hermitian 
metric on $T X_{\text {reg. }}$. We consider the complex, Hermitian vector space $V:=T_{x} X$ with Hermitian form $h_{H, x}$ and write

$$
G:=\operatorname{Hol}\left(X_{\mathrm{reg}}, g_{H}\right)_{x} \subseteq \mathrm{U}\left(V, h_{H, x}\right) \text { and } G^{\circ}:=\operatorname{Hol}\left(X_{\mathrm{reg}}, g_{H}\right)_{x}^{\circ} \subseteq \mathrm{SU}\left(V, h_{H, x}\right)
$$

for the (restricted) holonomy, as in Reminder 2.6 on page 8.

4.2. Geodesic incompleteness. The following result implies that many of the standard arguments used in the study of manifolds with vanishing first Chern class are not at our disposal.

Proposition 4.2 (Geodesic incompleteness in the standard setting). In the standard Setting 4.1, assume that $X_{\mathrm{reg}} \neq X$. In other words, assume that $X$ does have non-trivial singularities. Then, $\left(X_{\mathrm{reg}}, g_{H}\right)$ is not geodesically complete.

Proof. The current $T_{H}$ has bounded potentials. The volume of $X_{\text {reg }}$ is therefore computed as

$$
\operatorname{Vol}\left(X_{\text {reg }}, g_{H}\right)=\int_{X_{\text {reg }}} \omega_{H}^{n}=[H]^{n} .
$$

In particular, the volume is finite. Using that $\left(X_{\mathrm{reg}}, g_{H}\right)$ has non-negative Ricci curvature, a result of Yau then asserts that $\left(X_{\text {reg }}, g_{H}\right)$ cannot be geodesically complete unless $X_{\text {reg }}$ is compact, [SY94, Cor. on page 25].

Remark. In general, it is not know whether the diameter of $\left(X_{\mathrm{reg}}, g_{H}\right)$ is finite or not. However, if $X$ admits a smoothing or a crepant resolution, the diameter $\operatorname{diam}\left(X_{\mathrm{reg}}, g_{H}\right)$ is proven to be finite by [Tos09] and [RZ11, App. B].

4.3. Real analytic structure. Since $\left(X_{\text {reg }}, g_{H}\right)$ is Einstein as a Riemannian manifold, a theorem of DeTurck-Kazdan implies that $X_{\text {reg }}$ admits a smooth atlas with real analytic transition functions such that $g_{H}$ is real analytic in each coordinate chart, see [Bes87, Thm. 5.26]. This has the following consequence.

Proposition 4.3 (Behaviour of restricted holonomy under restriction). In the standard Setting 4.1, if $U=U(x) \subseteq X_{\text {reg }}$ is any (analytically) open neighbourhood of $x$, then the restricted holonomy groups $G^{\circ}$ and $\operatorname{Hol}\left(U,\left.g_{H}\right|_{U}\right)_{x}^{\circ}$ agree.

Proof. Since $\left(X_{\mathrm{reg}}, g_{H}\right)$ is real-analytic, both groups in question equal the local holonomy group at $x$, [KN96a, Thm. 10.8 on p. 101].

4.4. Quasi-étale covers in the standard setting. Working in the standard setting, we will frequently try to simplify geometry by passing to a suitable quasi-étale cover - this could be an index-one cover, which simplifies the singularities, or the holonomy cover that will be introduced in Section 7. The present section introduces notation and recalls a number of basic facts that will later be used.

4.4.1. Standard notation for quasi-étale covers. Given a quasi-étale cover $\gamma: Y \rightarrow X$, we may use Theorem 3.3 to construct a Ricci-flat Kähler metric $Y_{\text {reg. The }}$. Thiversal property of the EGZ construction, Proposition 3.5, will then allow to compare this metric to the one that we have on $X$. The following notation will be used consistently throughout the paper.

Notation 4.4 (Standard notation for quasi-étale covers). In the standard Setting 4.1, let $\gamma: Y \rightarrow X$ be a quasi-étale cover and $y \in \gamma^{-1}(x)$ be a point lying over $x$. Writing $Y^{\circ}:=\gamma^{-1}\left(X_{\text {reg }}\right)$, the following commutative diagram summarises the 
situation,

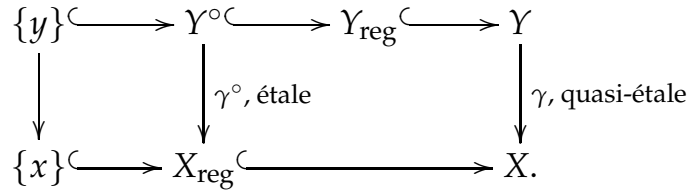

Recalling from Reminder 2.16 that $Y$ is klt and that $K_{Y}=\gamma^{*} K_{X}$ is numerically trivial, Theorem 3.3 applies to $\left(Y, H_{Y}\right)$ where $H_{Y}:=\gamma^{*} H$, and defines a Ricci-flat

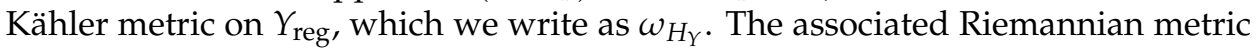
on $Y_{\text {reg }}$ will be written as $g_{H_{\gamma}}$, the associated Hermitian metric $h_{H_{\gamma}}$. We consider the complex, Hermitian vector space $V_{Y}:=T_{y} Y$ with Hermitian form $h_{H_{Y}, y}$, as well as the following subgroups of $\mathrm{U}\left(V_{Y}, h_{H_{Y}, y}\right)$,

$$
\begin{aligned}
I:=\operatorname{Hol}\left(Y^{\circ}, g_{H_{Y}}\right)_{y} \subseteq \operatorname{Hol}\left(Y_{\mathrm{reg}}, g_{H_{Y}}\right)_{y}=: G_{Y} \quad \text { and } \\
I^{\circ}:=\operatorname{Hol}\left(Y^{\circ}, g_{H_{Y}}\right)_{y}^{\circ} \subseteq \operatorname{Hol}\left(Y_{\mathrm{reg}}, g_{H_{Y}}\right)_{y}^{\circ}=: G_{Y}^{\circ} .
\end{aligned}
$$

The universal property of the EGZ construction, Proposition 3.5, asserts that

$$
\left.g_{H_{Y}}\right|_{\gamma^{\circ}}=\left(\gamma^{\circ}\right)^{*} g_{H} \text { and }\left.\omega_{H_{Y}}\right|_{\gamma^{\circ}}=\left(\gamma^{\circ}\right)^{*} \omega_{H} \text {. }
$$

In particular, we may use the isomorphism $\left.d \gamma^{\circ}\right|_{y}$ to identify the Hermitian vector spaces $V_{Y}$ and $V$, and to view $I, G_{Y}, I^{\circ}$ and $G_{Y}^{\circ}$ as subgroups of $\mathrm{U}\left(V, h_{H, x}\right)$.

4.4.2. Behaviour of holonomy under covers. We conclude the present section by pointing out a few relations between the groups introduced in Notation 4.4. The proof of the following elementary fact is left to the reader.

Fact 4.5 (Behaviour of holonomy under covers, I). In the setting of Notation 4.4, the following diagram is commutative

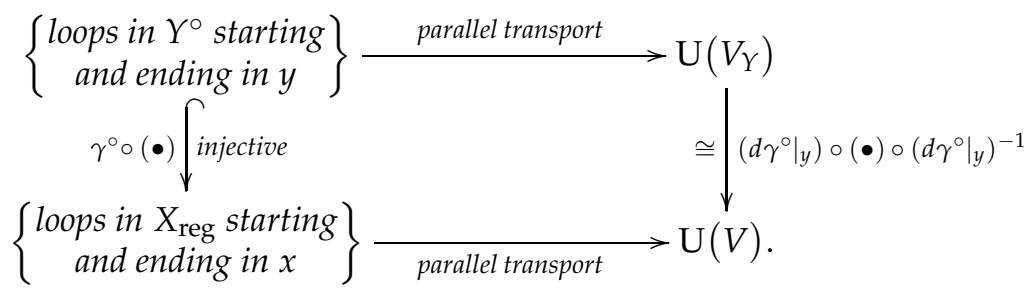

Remark 4.6 (Behaviour of holonomy under covers, II). In the setting of Notation 4.4, identifying $\mathrm{U}\left(V_{Y}\right)$ with $\mathrm{U}(V)$ using the isomorphism of Diagram (4.5.1), the diagram allows to view I as a subgroup of $G$. More precisely,

$$
\begin{aligned}
& I=\left\{g \in G \mid g \text { is parallel transport along a loop in } X_{\text {reg }}\right. \\
& \text { with homotopy class in } \left.\left(\gamma^{\circ}\right)_{*} \pi_{1}\left(Y^{\circ}, y\right)\right\} .
\end{aligned}
$$

This description together with Reminder 2.5 presents $I$ as a union of connected components of $G$ and therefore shows that the maximal connected subgroups agree, $I^{\circ}=G^{\circ}$.

Lemma 4.7 (Behaviour of holonomy under quasi-étale coverings, I). In the setting of Notation 4.4, the natural inclusions (4.4.1) and (4.4.2) are equalities. In other words, $I^{\circ}=G_{Y}^{\circ}$ and $I=G_{Y}$. 
Proof. The equality $I^{\circ}=G_{Y}^{\circ}$ has been shown in Proposition 4.3 above. To prove that $I=G_{Y}$, observe that $Y^{\circ}$ is a big subset of $Y_{\text {reg. The corresponding funda- }}$ mental groups therefore agree. We obtain a commutative diagram

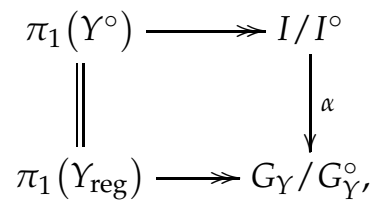

which implies that $\alpha$ is surjective. Using that $I^{\circ}=G_{\gamma}^{\circ}$, an application of the Snake Lemma then yields the desired equality $I=G_{Y}$.

Corollary 4.8 (Behaviour of holonomy under quasi-étale coverings, II). In the setting of Notation 4.4, viewing $G_{Y}$ as a subgroup of $\mathrm{U}\left(V, h_{H, x}\right)$, we have equality $G^{\circ}=G_{Y}^{\circ}$ and an inclusion $G_{Y} \subseteq G$ that is given as follows,

$$
\begin{array}{r}
G_{Y}=\left\{g \in G \mid g \text { is parallel transport along a loop in } X_{\mathrm{reg}}\right. \\
\text { with homotopy class in } \left.\left(\gamma^{\circ}\right)_{*} \pi_{1}\left(Y^{\circ}, y\right)\right\} .
\end{array}
$$

Proof. Consider the groups $I^{\circ}$ and $I$. Remark 4.6 immediately identifies $G^{\circ}$ with $I^{\circ}$, and the right hand side of (4.8.1) with $I$. The identifications of Lemma 4.7 thus end the proof.

4.5. Parallel transport of invariant subspaces. The following theorem asserts that parallel subbundles of $T X_{\text {reg }}$ and $T^{*} X_{\text {reg }}$ are holomorphic. We use this result in Section 6 to find a canonical direct sum decomposition in the sheaf of reflexive forms, which relates (strong) stability with irreducibility properties of the (restricted) holonomy action and explains the behaviour of these properties under quasiétale coverings.

Proposition 4.9 (Parallel transport of invariant subspaces). In the standard Setting 4.1, let $N \subseteq V$ be any G-invariant linear subspace, and $\widehat{N} \subseteq T X_{\text {reg }}$ be the associated parallel, smooth subbundle obtained by parallel transport. Then, $\widehat{N}$ is a holomorphic subbundle of $T X_{\mathrm{reg}}$. Ditto for subbundles of $T^{*} X_{\mathrm{reg}}$.

Proof. Consider $\left(T X_{\text {reg }}, h_{H}\right)$ as a holomorphic Hermitian vector bundle and apply [Kob87, Chap. I, Prop. 4.18] to $\widehat{N}$ in order to get the desired result.

The result above can also be expressed as a statement about sheaves.

Corollary 4.10 (Holonomy principle for bundles as a statement about sheaves). In the standard Setting 4.1, let $N \subseteq V$ be any G-invariant linear subspace, and $\widehat{N} \subseteq T X_{\text {reg }}$ be the associated parallel, smooth subbundle obtained by parallel transport. Then, there exists a locally free subsheaf of $\mathscr{O}_{X_{\mathrm{reg}}}$-modules $j: \mathscr{N}^{\circ} \hookrightarrow \mathscr{T}_{\mathrm{X}_{\mathrm{reg}}}$ with locally free quotient, and a commutative diagram

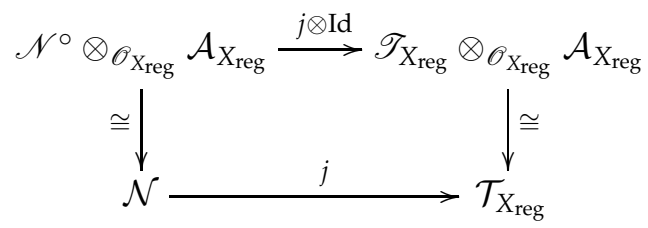

where $\mathcal{N}$ denotes the sheaf of smooth sections of $\widehat{N}$. Ditto for subbundles of $T^{*} X_{\text {reg. }}$. 


\section{Part II. Holonomy}

\section{THE CLASSIFICATION OF RESTRICTED HOLONOMY}

After fixing our notation regarding the restricted holonomy decomposition, we apply the standard classification of irreducible restricted holonomy groups appearing in our setup.

5.1. Notation. The following construction and notation will be used throughout.

Construction and Notation 5.1 (Decomposition induced by restricted holonomy). Assume the Standard Setting 4.1 and recall from [Bes87, Cor. 10.41] that the action $G^{\circ} \circlearrowleft V$ is totally decomposed. That is, there exist decompositions

$$
V=V_{0} \oplus V_{1} \oplus \cdots \oplus V_{m} \quad \text { and } \quad G^{\circ}=G_{1}^{\circ} \times \cdots \times G_{m}^{\circ},
$$

where each factor $G_{i}^{\circ}$ acts non-trivially and irreducibly on $V_{i}$ and trivially on all the other summands. We refer to (5.1.1) as the canonical decomposition of the Hermitian $G^{\circ}$-space $V$. The induced decomposition of the dual space $V^{*}=T_{x}^{*} X$ will analogously be called canonical decomposition of the Hermitian $G^{\circ}$-space $V^{*}$.

Remark 5.2 (Behaviour under quasi-étale covers). If $\gamma: Y \rightarrow X$ is a quasi-étale cover, and $y \in \gamma^{-1}(x)$ is any point, recall from Corollary 4.8 that $V=T_{x} X$ and $V_{Y}=T_{y} Y$ are canonically identified, and that the restricted holonomy groups agree, $G^{\circ}=G_{Y}^{\circ}$. In this sense, the canonical decomposition of Construction 5.1 is invariant under passing to quasi-étale covers.

The action $G^{\circ} \circlearrowleft V$ carries algebro-geometric information: We will see in Corollary 7.2 that the dimension of $V_{0}$ equals the augmented irregularity $\widetilde{q}(X)$. Furthermore, we will see in Corollary 7.4 that $\mathscr{T}_{X}$ is strongly stable if and only if the action $G^{\circ} \circlearrowleft V$ is irreducible.

5.2. Classification. Using Proposition 2.9, Proposition 4.3, as well as Berger's classification [Bes87, Thm. 10.108], one easily obtains the following result.

Proposition 5.3 (Classification of restricted holonomy). Setting and notation as in 5.1. Given any index $1 \leq i \leq m$ and writing $n_{i}:=\operatorname{dim} V_{i}$, one of the following holds true.

(5.3.1) The group $G_{i}^{\circ}$ is isomorphic to $\mathrm{SU}\left(n_{i}\right)$.

(5.3.2) The number $n_{i}$ is even, and the group $G^{\circ}$ is isomorphic to $\operatorname{Sp}\left(\frac{n_{i}}{2}\right)$.

The action $G_{i}^{\circ} \circlearrowleft V_{i}$ is isomorphic to the standard action of the respective group.

Proof. Choose a simply connected open neighbourhood $W=W(x) \subseteq X_{\text {reg. Ap- }}$ plying Proposition 2.9 ("Local decomposition of Kähler manifolds") to $\left(W,\left.\omega_{H}\right|_{W}\right)$, we obtain a neighbourhood $U=U(x) \subseteq W$, Kähler manifolds $\left(U_{i}, \omega_{i}\right)$ and an identification of $\left(U, \omega_{H} \mid U\right)$ with the product of Kähler manifolds, $\left(U_{i}, \omega_{i}\right) \times \cdots \times$ $\left(U_{m^{\prime}}, \omega_{m^{\prime}}\right)$. Since $\left(U,\left.\omega_{H}\right|_{U}\right)$ is Ricci-flat, the same will hold for all the $\left(U_{i}, \omega_{i}\right)$. We maintain the notation of Proposition 2.9 for the remainder of the proof. Shrinking the $U_{i}$ if necessary, we can assume without loss of generality that all $U_{i}$ as well as $U$ are simply connected. We will write $G_{0}^{\circ}$ for the trivial group.

Proposition 2.9 also asserts that the action of the (restricted) holonomy group $H:=\operatorname{Hol}\left(U,\left.g_{H}\right|_{U}\right)_{x}=\operatorname{Hol}\left(U,\left.g_{H}\right|_{U}\right)_{x}^{\circ}$ on $V=T_{x} U$ is totally decomposed. The associated decompositions of $H$ and $T_{x} U$ are given as follows,

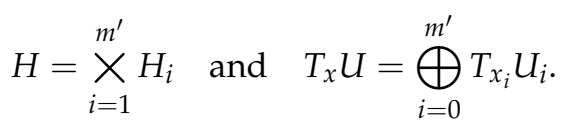




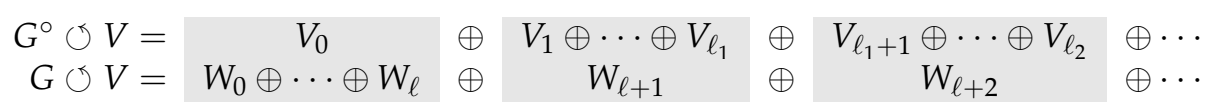

FIGURE 6.1. Decompositions induced by restricted and full holonomy

Recall from Proposition 4.3 ("Behaviour of restricted holonomy under restriction") that the natural inclusion $H \subseteq G^{\circ}$ is in fact an equality. We observed in Remark 2.12 that any two decompositions of $G^{\circ} \circlearrowleft V$ agree up to permutation, which shows that $m=m^{\prime}$. Renumbering the $U_{i}$ if needed, we may even assume that $V_{i}=T_{x_{i}} U_{i}$ and $H_{i}=G_{i}^{\circ}$ for all $i$. In summary, we see that $\left(U_{0}, \omega_{0}\right)$ is flat, whereas all other $\left(U_{i}, \omega_{i}\right)_{i>0}$ are Ricci-flat, holonomy-irreducible Kähler manifolds. The description of the $G_{i}^{\circ}=\operatorname{Hol}\left(U_{i}, g_{i}\right)_{x_{i}}^{\circ}$ is now a standard consequence of the classification of non-symmetric, restricted holonomy groups, [Bes87, Thm. 10.108], and the fact that Ricci-flat locally symmetric spaces are flat, [Sim62, Cor. on p. 232].

\section{THE CANONICAL DECOMPOSITION OF THE TANGENT SHEAF}

In this section, we use standard holonomy considerations to obtain a canonical decomposition of the tangent sheaf. This decomposition generalises both [GKP16b, Thm. 1.3] and [Gue16, Thm. A], and yields a differential-geometric characterisation of stability for the tangent bundle, Corollary 6.8 .

6.1. Construction of the decomposition. Just like the action of the restricted holonomy, the action of the full holonomy group $G \circlearrowleft V$ is totally decomposed and induces a canonical decomposition of $V$.

Construction and Notation 6.1 (Decomposition induced by holonomy). Assume the Standard Setting 4.1 and recall from [Bes87, Cor. 10.38] that the action $G \circlearrowleft V$ is totally decomposed. That is, there exist decompositions

$$
V=W_{0} \oplus W_{1} \oplus \cdots \oplus W_{k} \text { and } G=G_{1} \times \cdots \times G_{k}
$$

where each factor $G_{i}$ acts non-trivially and irreducibly on $W_{i}$ and trivially on all the other summands. As before, we abuse notation and refer to (6.1.1) as the canonical decomposition of the Hermitian $G$-space $V$. We denote the associated smooth, parallel bundles by $\widehat{W}_{j} \subseteq T X_{\text {reg. }}$.

We have seen in Remark 2.12 that decomposition (6.1.1) is unique up to permutation of the $W_{j}$ and $G_{j}$ of positive index. We now fix one choice of indices.

Observation and Notation 6.2 (Comparison of the canonical decompositions). Assume the Standard Setting 4.1 and recall that $G^{\circ}$ is a normal subgroup of $G$. This implies that elements of $G$ map $G^{\circ}$-orbits to $G^{\circ}$-orbits. The description of the $V_{i}$ found in Construction 5.1 therefore implies that any element of $G$ stabilises $V_{0}$, and permutes the remaining $\left(V_{i}\right)_{i>0}$. Renumbering the $V_{i}$ and $W_{j}$, if necessary, we may therefore assume without loss of generality that there exists an index $\ell$ and a strictly increasing sequences of indices $0=\ell_{0}<\ell_{1}<\ell_{2}<\cdots$ such that the following holds,

(6.2.1) The space $V_{0}$ decomposes as $V_{0}=W_{0} \oplus \cdots \oplus W_{\ell}$.

(6.2.2) For every positive $j$, we have $W_{\ell+j}=V_{\ell_{j-1}+1} \oplus \cdots \oplus V_{\ell_{j}}$. The summands are isomorphic as $G^{\circ}$-representation spaces.

Figure 6.1 illustrates the setting.

Remark 6.3 (Behaviour under quasi-étale covers). Continuing Remark 5.2, if $\gamma$ : $Y \rightarrow X$ is a quasi-étale cover, and $y \in \gamma^{-1}(x)$ is any point, recall from Corollary 4.8 
that $V=T_{x} X$ and $V_{Y}=T_{y} Y$ are canonically identified, and that the holonomy group $G_{Y}$ is a subgroup of $G$ under this identification. In this sense, the canonical decomposition on $Y$ refines the canonical decomposition on $X$.

6.2. The decomposition the tangent sheaf. The holonomy principle for bundles immediately yields a canonical direct sum decomposition of the tangent.

Construction and Notation 6.4 (Canonical decomposition of $\mathscr{T}_{X}$ ). Assume the setup of Construction and Notation 6.1. If $\mathcal{W}_{j}$ denotes the sheaf of smooth sections of $\widehat{W}_{j} \subseteq T X_{\text {reg, }}$, the holonomy principle for bundles, Corollary 4.10 , yields a decomposition $\mathscr{T}_{\text {reg }}=\mathscr{W}_{0}^{\circ} \oplus \cdots \oplus \mathscr{W}_{k}^{\circ}$ in the category of coherent analytic sheaves such that

$$
\mathcal{T}_{X_{\text {reg }}} \cong \mathcal{W}_{0} \oplus \cdots \oplus \mathcal{W}_{k} \cong\left(\mathscr{W}_{0}^{\circ} \otimes_{\mathscr{O}_{X}} \mathcal{A}_{X_{\text {reg }}}\right) \oplus \cdots \oplus\left(\mathscr{W}_{k}^{\circ} \otimes_{\mathscr{O}_{X}} \mathcal{A}_{X_{\text {reg }}}\right)
$$

Writing $\mathscr{W}_{i}:=\iota_{*} \mathscr{W}_{i}^{\circ}$, where $\iota: X_{\text {reg }} \rightarrow X$ is the inclusion, we obtain a splitting

$$
\mathscr{T}_{X}=\mathscr{W}_{0} \oplus \cdots \oplus \mathscr{W}_{k}
$$

As a direct summand of a coherent analytic sheaf, each one of the analytic sheaves $\mathscr{W}_{i}$ is coherent and hence algebraic by GAGA. Moreover, each $\mathscr{W}_{i}$ is reflexive by construction. Write $\mathscr{V}_{0}^{\circ}:=\mathscr{W}_{0}^{\circ} \oplus \cdots \oplus \mathscr{W}_{\ell}^{\circ}$ and $\mathscr{V}_{0}:=\iota_{*} \mathscr{V}_{0}^{\circ}$. We refer to (6.4.1) as the canonical decomposition of $\mathscr{T}_{X}$.

Remark 6.5 (Behaviour under quasi-étale covers). If $\gamma: Y \rightarrow X$ is a quasi-étale cover, there are now two decompositions on $Y$ : the canonical decomposition on $Y$, and the reflexive pull-back of the canonical decomposition on $X$,

$$
\begin{aligned}
\mathscr{T}_{Y} & =\mathscr{W}_{Y, 0} \oplus \cdots \oplus \mathscr{W}_{Y, \ell_{Y}} \oplus \mathscr{W}_{Y, \ell_{Y}+1} \oplus \cdots \oplus \mathscr{W}_{Y, k_{Y}} \\
& =\gamma^{[*]} \mathscr{W}_{0} \oplus \cdots \oplus \gamma^{[*]} \mathscr{W}_{\ell} \oplus \gamma^{[*]} \mathscr{W}_{\ell+1} \oplus \cdots \oplus \gamma^{[*]} \mathscr{W}_{k} .
\end{aligned}
$$

Remark 6.3 implies that (6.5.1) refines (6.5.2). Remark 5.2 implies that

$$
\gamma^{[*]} \mathscr{V}_{0}=\gamma^{[*]} \mathscr{W}_{0} \oplus \cdots \oplus \gamma^{[*]} \mathscr{W}_{\ell} \quad \text { and } \quad \mathscr{V}_{Y, 0}=\mathscr{W}_{Y, 0} \oplus \cdots \oplus \mathscr{W}_{Y, \ell_{Y}}
$$

agree.

We conclude the present subsection with a first description of the summands that appear in the decomposition. Once the Bochner principle for reflexive tensors is established in Theorem 8.2, we will be able say more, see Corollary 8.4.

Proposition 6.6 (Summands in the canonical decomposition of $\mathscr{T}_{X}$ ). In the setting of Construction 6.4, the following holds.

(6.6.1) The Hermitian holomorphic vector bundles $\mathscr{W}_{0}^{\circ}, \ldots, \mathscr{W}_{\ell}^{\circ}$ are unitary flat. In particular, the locally free sheaves $\mathscr{W}_{0}^{\circ}, \ldots, \mathscr{W}_{\ell}^{\circ}$ and $\mathscr{V}_{0}^{\circ}$ are holomorphically flat.

(6.6.2) If $\widehat{F} \subseteq T X_{\text {reg }}$ is any parallel subbundle and $i>0$ is any index, then the projection map $p_{i}: F \rightarrow W_{i}$ at $x$ is either zero or surjective.

(6.6.3) The locally free sheaf $\mathscr{W}_{0}^{\circ}$ is holomorphically trivial and if $i>0$ is any index, then $\mathscr{W}_{i}$ is stable of slope zero with respect to any ample polarisation on $X$. In particular, $\mathscr{T}_{X}$ is polystable with respect to any ample polarisation on $\mathrm{X}$.

Remark 6.7 (Non-flatness of remaining summands). Improving on Item (6.6.1), we will later see in Corollary 7.2 that none of the remaining summands $\mathscr{W}_{\ell+1}^{\circ}, \ldots, \mathscr{W}_{k}^{\circ}$ is holomorphically flat.

Proof of Proposition 6.6. - 
Proof of Item (6.6.1). Choose an integer $0 \leq j \leq \ell$. As the holomorphic vector bundle $\mathscr{W}_{j}^{\circ}$ is obtained by parallel transport, it is a direct summand of $\mathscr{T}_{\text {reg }}$ as a Hermitian holomorphic subbundle. Furthermore, $\mathscr{W}_{j}^{\circ}$ is acted trivially upon by the restricted holonomy group $G^{\circ}$, and hence its restricted holonomy group is trivial. Shrinking to small simply connected neighbourhoods, one can therefore find parallel local frames for $\mathscr{W}_{j}^{\circ}$. It follows that the Hermitian structure of $\mathscr{W}_{j}^{\circ}$ is flat, and the (1,0)-part of the Chern connection is a flat holomorphic connection.

Proof of Item (6.6.2). Parallel transport stabilises both $\widehat{F}$ and $\widehat{W}_{i}$, and commutes with the projection map $\mathrm{pr}_{i}$. As a consequence, it follows that the rank of $\mathrm{pr}_{i}$ is constant. To prove surjectivity, it will therefore suffice to show surjectivity at the point $x$. In other words, writing $F:=\left.\widehat{F}\right|_{\{x\}}$, we need to show surjectivity of the composition

$$
F \underset{\text { inclusion }}{\longrightarrow} V=W_{0} \oplus \cdots \oplus W_{k} \underset{\text { projection }}{\longrightarrow} W_{i} .
$$

The morphisms in (6.7.1) are linear maps of G-representation spaces. Since the representation space $W_{i}$ is irreducible by assumption, any equivariant map with image in $W_{i}$ must either be zero, or surjective. Item (6.6.2) follows.

Proof of Item (6.6.3). The triviality of $\mathscr{W}_{0}^{\circ}$ is clear, as one can parallel transport any basis of $W_{0}$ to obtain a trivialising set of holomorphic sections of $\mathscr{W}_{0}^{\circ}$, cf. Reminder 2.7. Now, recall from [Gue16, Thm A.(ii)] that there exists a decomposition $\mathscr{T}_{X}=\oplus \mathscr{F}_{j}$ with the following properties.

(6.7.2) The $\mathscr{F}_{j}$ are reflexive, and stable of slope zero with respect to any ample polarisation of $X$.

(6.7.3) The restrictions $\mathscr{F}_{j}^{\circ}:=\left.\mathscr{F}_{j}\right|_{X_{\text {reg }}}$ are locally free. The associated subbundles of $F_{j}^{\circ} \subseteq T X_{\text {reg }}$ are parallel ${ }^{2}$.

In particular, it follows that $\mathscr{T}_{X}$ is semistable of slope zero with respect to any polarisation, and hence so are the direct summands $\mathscr{W}_{\bullet}$ in the canonical decomposition. Now, given any index $i>0$, we will show that $\mathscr{W}_{i}$ is isomorphic to one of the $\mathscr{F}_{j}$, hence stable with respect to any polarisation. We start by choosing an index $j$ such that the projection map $p_{j i}:=\left.\mathrm{pr}_{i}\right|_{\mathscr{F}_{j}}: \mathscr{F}_{j} \rightarrow \mathscr{W}_{i}$ is not zero.

As a non-trivial map from a stable sheaf to a semistable sheaf of the same slope, $p_{j i}$ is clearly injective. We claim that $p_{j i}$ is also surjective. Since both $\mathscr{F}_{j}$ and $\mathscr{W}_{i}$ are reflexive, it suffices to show surjectivity of the restricted map $p_{j i}^{\circ}: \mathscr{F}_{j}^{\circ} \rightarrow \mathscr{W}_{i}^{\circ}$. That, however, has been established in Item (6.6.2) above.

6.3. Canonical decomposition vs. earlier results. Next, we explain how the canonical decomposition of $\mathscr{T}_{X}$ relates to earlier work, and how its uniqueness improves known results.

6.3.1. Uniqueness. The decomposition of a polystable sheaf into stable summands is unique up to non-canonical isomorphism, but not unique in general. However, as soon as a singular Ricci-flat Kähler metric is fixed, the canonical decomposition of $\mathscr{T}_{X}$ is unique up to permutation of the factors $\mathscr{W}_{1}, \ldots, \mathscr{W}_{k}$, as follows from Remark 2.12. The factor $\mathscr{W}_{0}$, although unique, is not stable as soon as its rank is larger than one, and it does not admit a unique decomposition into stable subsheaves.

\footnotetext{
${ }^{2}$ This is shown in the proof of [Gue16, Thm. A(ii)], on page 35, a few lines ahead of $\S 5$.
} 
6.3.2. Comparison with earlier results. It follows from the discussion above that the polystability decomposition of [Gue16, Thm. A] in case $K_{X} \equiv 0$ is isomorphic to the canonical decomposition of $\mathscr{T}_{X}$, unless there exists a trivial summand of rank at least two.

Moreover, the proof of Item (6.6.3) above implies that on the quasi-étale cover $\gamma: Y \rightarrow X$ whose existence is established in [GKP16b, Thm. 1.3] the summands of the decomposition of the tangent sheaf $\mathscr{T}_{Y}$ produced by loc. cit. are isomorphic to the ones in the canonical decomposition (6.4.1) of $\mathscr{T}_{Y}$. In particular, the summands in the canonical decomposition of $\mathscr{T}_{Y}$ are strongly stable in the sense of [GKP16b, Def. 7.2] and have trivial determinant. The latter property furthermore implies integrability by [GKP16b, Thm. 7.11].

6.4. Stability and irreducibility of the holonomy representations. The canonical decomposition relates stability of $\mathscr{T}_{X}$ to irreducibility of the holonomy representation. The following corollaries are immediate consequences of Proposition 6.6. Later, Corollary 7.4 will also relate strong stability and irreducibility of $G^{\circ} \circlearrowleft V$.

Corollary 6.8 (Stability and irreducibility, I). In the standard Setting 4.1, the following statements are equivalent.

(6.8.1) The sheaf $\mathscr{T}_{X}$ is stable with respect to any ample polarisation.

(6.8.2) The holonomy representation $G \circlearrowleft V$ is irreducible.

Proposition 6.6 also applies to describe the holonomy representation in case where $\mathscr{T}_{X}$ is stable only with respect to a movable curve class, or with respect to nef divisors. Stability with respect to a movable class is discussed in the paper [GKP16a]. We refer to [GKP16a, Def. 2.11] for a precise definition.

Corollary 6.9 (Stability and irreducibility, II). In the standard Setting 4.1, assume that one of the following holds.

(6.9.1) There exist nef Cartier divisors $H_{1}, \ldots, H_{n-1}$ on $X$ such that $\mathscr{T}_{X}$ is stable with respect to $\left(H_{1}, \ldots, H_{n-1}\right)$.

(6.9.2) The variety $X$ is $Q$-factorial, and there exists a movable curve class $\alpha \in N_{1}(X)_{\mathbb{R}}$ such that $\mathscr{T}_{X}$ is $\alpha$-stable.

Then, the holonomy representation $G \circlearrowleft V$ is irreducible.

Proof. We discuss case (6.9.2) only; the other case is completely similar. If the holonomy representation is reducible, Construction 6.4 yields a proper decomposition $\mathscr{T}_{X}=\mathscr{W}_{0} \oplus \cdots \oplus \mathscr{W}_{k}$, with $k>0$ or $k=0$ and $\operatorname{dim} \mathscr{W}_{0}>1$. In the second case, $\mathscr{T}_{X}$ is trivial, contradiction. In the first case, stability of $\mathscr{T}_{X}$ with respect to $\alpha$ implies the slope inequality $\mu_{\alpha}\left(\mathscr{W}_{i}\right)<\mu_{\alpha}\left(\mathscr{T}_{X}\right)=0$ for all $i$, which contradicts the identity

$$
0=\mu_{\alpha}\left(\mathscr{T}_{X}\right)=\sum_{i=1}^{k} \frac{\operatorname{rank}\left(\mathscr{W}_{i}\right)}{n} \cdot \mu_{\alpha}\left(\mathscr{W}_{i}\right)
$$

\section{COVERING CONSTRUCTIONS}

7.1. Main result. The quotient $G / G^{\circ}$ frequently appears as an obstruction to extending locally defined parallel tensors to global objects. The difference between holonomy and restricted holonomy clearly goes away once we pass to the universal covering space of $X_{\text {reg }}$, but this comes at the price of potentially losing all algebraic structures, as we have no a priori information on the fundamental group of $X_{\text {reg. }}$. The following result, which deals with this issue, is crucial for all our subsequent arguments and is therefore one of the main results of this paper. 


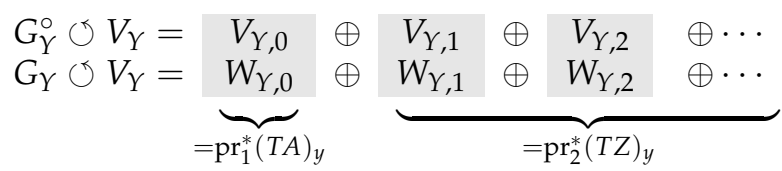

FIGURE 7.1. Canonical decompositions on a holonomy cover

Theorem and Notation 7.1 (Holonomy cover). In the standard Setting 4.1, there exists a quasi-étale cover $\gamma: Y \rightarrow X$ and a point $y \in \gamma^{-1}(x)$, such that holonomy and restricted holonomy agree, $G_{Y}^{\circ}=G_{Y}$. Further, there exist normal, projective varieties $A$ and $Z$ and an isomorphism $Y \cong A \times Z$, such that the following additional properties hold.

(7.1.1) The variety $A$ is Abelian, of dimension $\operatorname{dim} A=\widetilde{q}(X)$.

(7.1.2) The variety $Z$ has canonical singularities, trivial canonical bundle, and augmented irregularity $\widetilde{q}(Z)=0$.

(7.1.3) The summands $V_{Y, 0}$ and $W_{Y, 0}$ of $V_{Y}$ both coincide with $\left.\operatorname{pr}_{1}^{*}(T A)\right|_{y}$.

(7.1.4) The summand $W_{Y, 1} \oplus \cdots \oplus W_{Y, k}$ of $V_{Y}$ coincides with $\left.\operatorname{pr}_{2}^{*}(T Z)\right|_{y}$.

Figure 7.1 illustrates the canonical decompositions of $V_{Y}$. Quasi-étale covers with these properties will be called holonomy covers.

Theorem 7.1 has a number of consequences for the holonomy, augmented irregularity and canonical decomposition of the initial variety. The following might be of independent interest.

Corollary 7.2 (Consequences for the standard setting). In the standard Setting 4.1, the following will hold.

(7.2.1) The quotient group $G / G^{\circ}$ is finite. In particular, the factors $G_{1}, \ldots, G_{\ell}$ of the holonomy group $G$ are all finite.

(7.2.2) The augmented irregularity $\widetilde{q}(X)$ equals $\operatorname{dim} V_{0}$.

(7.2.3) None of the summands $\mathscr{W}_{\ell+1}^{\circ}, \ldots, \mathscr{W}_{k}^{\circ}$ is holomorphically flat.

Theorem 7.1 will be shown in Section 7.4. Corollary 7.2 is proven immediately afterwards in Section 7.5. To prepare for the proof of Theorem 7.1, Sections 7.2 and 7.3 construct covers that realise partial aspects of the holonomy cover. Combining all results obtained thus far, Section 7.6 proves Proposition D.

7.2. The weak holonomy cover. The canonical decomposition of $V$ induced by the holonomy group $G$ does not in general refine the decomposition induced by restricted holonomy $G^{\circ}$, see Figure 6.1 on page 19 for an illustration. The following result shows that this problem vanishes once we pass to a suitable quasi-étale cover.

Proposition and Notation 7.3 (Weak holonomy cover). In the standard Setting 4.1, there exists a quasi-étale cover $\gamma: Y \rightarrow X$ and a point $y \in \gamma^{-1}(x)$, such that the following holds.

(7.3.1) Using Notation 4.4, the holonomy action $G_{Y} \circlearrowleft V_{Y}$ stabilises the canonical decomposition of $V_{Y}$ that is induced by the restricted holonomy action $G_{Y}^{\circ} \circlearrowleft V_{Y}$. In particular, the canonical decomposition induced by $G_{Y}$ is a refinement of the canonical decomposition induced by $G_{Y}^{\circ}$.

(7.3.2) A quasi-étale cover of pointed spaces satisfies (7.3.1) if and only if it admits a factorisation via $\gamma$. In this case, the factorisation is unique. In particular, the covering $\gamma$ is unique up to canonical isomorphism.

Figure 7.2 on the following page illustrates the canonical decompositions of $V_{Y}$. We refer to the covering $\gamma$ as the weak holonomy cover. 


$$
\begin{array}{lc|c|c|c|c|c}
G_{Y}^{\circ} V_{Y}= & V_{Y, 0} & \oplus & V_{Y, 1} & \oplus & V_{Y, 2} & \oplus \cdots \\
G_{Y} \circlearrowleft V_{Y}= & W_{Y, 0} \oplus \cdots \oplus W_{Y, \ell_{Y}} & \oplus & W_{Y, \ell_{Y}+1} & \oplus & W_{Y, \ell_{Y}+2} & \oplus \cdots
\end{array}
$$

FIGURE 7.2. Canonical decompositions on the weak holonomy cover

Proof. Write $V=V_{0} \oplus \cdots \oplus V_{m}$ for the canonical decomposition of $V$ induced by the action of $G^{\circ}$. We have seen in Observation 6.2 that every element $g \in G$ stabilises $V^{0}$ and permutes the remaining summands. From this and from Reminder 2.5 we obtain a morphism

$$
\pi_{1}\left(X_{\text {reg }}, x\right) \stackrel{\sigma}{\longrightarrow} \underset{\longrightarrow}{\longrightarrow} \longrightarrow \text { Permutations }\{1, \ldots, m\} .
$$

Recalling from Remark 2.17 that there exists an equivalence between quasi-étale covers and finite sets with transitive action of $\pi_{1}\left(X_{\mathrm{reg}}, x\right)$, the morphism (7.3.3) thus gives the desired cover.

For the next corollary, recall that a reflexive sheaf is "strongly stable" if its reflexive pull-back to any quasi-étale cover is stable with respect to any polarisation $\left(H_{1}, \ldots, H_{n-1}\right)$ there. We refer to [GKP16b] for a more detailed discussion, in particular concerning the role of varieties with strongly stable tangent sheaf in the structure theory of varieties with numerically trivial canonical divisor.

Corollary 7.4 (Strong stability and irreducibility of restricted holonomy). In the standard Setting 4.1, the following assertions are equivalent.

(7.4.1) The sheaf $\mathscr{T}_{X}$ is strongly stable.

(7.4.2) The restricted holonomy representation $G^{\circ} \circlearrowleft V$ is irreducible.

Proof of Corollary 7.4. The implications are proven separately.

Implication (7.4.1) $\Rightarrow$ (7.4.2). Consider a weak holonomy cover $\gamma: Y \rightarrow X$, as constructed in Proposition 7.3. Assuming (7.4.1), the sheaf $\mathscr{T}_{Y}$ will then be stable with respect to any ample polarisation. By Corollary 6.8, this implies that the holonomy action $G_{Y} \circlearrowleft V_{Y}$ is irreducible. By choice of $Y$, either the restricted holonomy $G_{Y}^{\circ}$ is irreducible or it is trivial. Identifying $V$ and $V_{Y}$ as explained in Notation 4.4, we have seen in Corollary 4.8 that the groups $G^{\circ}$ and $G_{Y}^{\circ}$ are naturally identified, and that their natural representations $G^{\circ} \circlearrowleft V$ and $G_{Y}^{\circ} \circlearrowleft V_{Y}$ are equivalent.

If the restricted holonomy $G_{Y}^{\circ}$ is irreducible, then so is $G^{\circ}$ and we are done. If $G_{Y}^{\circ}$ is trivial, then so is $G^{\circ}$ and $\mathscr{T}_{X_{\text {reg }}}$ is flat. By [GKP16c, Cor. 1.16], there exists a finite, quasi-étale cover $A \rightarrow X$ where $A$ is an abelian variety. As $\operatorname{dim} X \geq 2, \mathscr{T}_{X}$ is not strongly stable, a contradiction. The implication (7.4.1) $\Rightarrow$ (7.4.2) follows.

Implication $\neg(7.4 .1) \Rightarrow \neg(7.4 .2)$. We start the proof by constructing a sequence of quasi-étale coverings as follows,

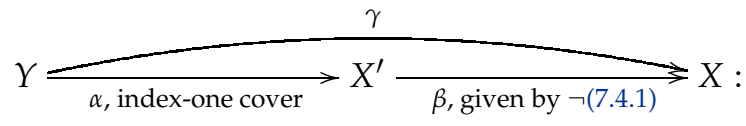

As we assume that $\mathscr{T}_{X}$ is not strongly stable, we find a quasi-étale cover $\beta: X^{\prime} \rightarrow X$ and ample divisors $H_{1}, \ldots, H_{n-1}$ on $X^{\prime}$ such that $\mathscr{T}_{X^{\prime}}$ is not stable with respect to $\left(H_{1}, \ldots, H_{n-1}\right)$. The space $X^{\prime}$ is again klt, and its canonical class $K_{X^{\prime}}$ is again numerically trivial. Let $\alpha: Y \rightarrow X^{\prime}$ be a global index-one cover, whose existence is guaranteed by Proposition 2.18. Moreover, setting $H_{Y, i}:=\alpha^{*} H_{i}$, the sheaf $\mathscr{T}_{Y}=$ $\alpha^{[*]} \mathscr{T}_{X^{\prime}}$ is not stable with respect to $\left(H_{Y, 1}, \ldots, H_{Y, n-1}\right)$. Together with [GKP16b, 
Prop. 5.7] these properties imply that $\mathscr{T}_{Y}$ is in fact not stable with respect to any tuple of ample bundles. Corollary 6.8 therefore implies that the holonomy action $G_{Y} \circlearrowleft V_{Y}$ is not irreducible, and then neither is the action of the restricted holonomy group, $G_{Y}^{\circ} \circlearrowleft V_{Y}$. Using Corollary 4.8 to identify the representations $G^{\circ} \circlearrowleft V$ and $G_{Y}^{\circ} \circlearrowleft V_{Y}$, the implication $\neg(7.4 .1) \Rightarrow \neg(7.4 .2)$ thus follows.

7.3. Torus covers. We have seen in Proposition 6.6 that the first summands of the canonical decomposition, $\mathscr{W}_{0}, \ldots, \mathscr{W}_{\ell}$ are locally free on $X_{\text {reg, }}$, and are holomorphically flat there. The following theorem gives a geometric explanation for this observation.

Proposition and Notation 7.5 (Torus cover). In the standard Setting 4.1, there exist normal, projective varieties $A$ and $Z$, and a quasi-étale cover $\gamma: Y \rightarrow X$ such that $Y$ has a product structure, $Y=A \times Z$, with the following properties.

(7.5.1) The variety $A$ is Abelian, of dimension $\operatorname{dim} A=\widetilde{q}(X)$.

(7.5.2) The variety $Z$ has canonical singularities, trivial canonical bundle, and augmented irregularity $\widetilde{q}(Z)=0$.

A quasi-étale cover $Y \rightarrow X$ where $Y=A \times Z$ has a product structure with properties (7.5.1) and (7.5.2) will be called $a$ torus cover.

Proof. We construct a sequence of projective varieties and quasi-étale covers as follows,

$$
A \times Z \underset{\text { splitting off torus }}{\stackrel{c}{\longrightarrow}} X^{\prime \prime} \underset{\text { index-one }}{\stackrel{b}{\longrightarrow}} X^{\prime} \underset{\text { realising } \widetilde{q}}{\longrightarrow} X .
$$

As quasi-étale covers of $X$, all varieties will again be klt, with numerically trivial canonical class. To be more precise, let $a: X^{\prime} \rightarrow X$ be a quasi-étale cover that realises the augmented irregularity, $q\left(X^{\prime}\right)=\widetilde{q}(X)$. Such a cover exists by definition, because $\widetilde{q}(X)$ is finite by Item (2.21.2) of Lemma 2.21. Next, we consider a global index-one cover $b: X^{\prime \prime} \rightarrow X^{\prime}$, as given by Proposition 2.18. Finally, recall from [GKP16b, Thm. 1.3] that there exists an Abelian variety $A$, a canonical variety $Z$ with $\widetilde{q}(Z)=0$ and a quasi-étale cover $c: A \times Z \rightarrow X^{\prime \prime}$. Recalling from [Laz04, Lem. 4.1.14] ("Injectivity Lemma") that the irregularity increases in covers, the equality of augmented and actual irregularity still holds on $A \times Z$. In other words, $\widetilde{q}(X)=q(A \times Z)=\operatorname{dim} A$. We consider the composition $\gamma: A \times Z \rightarrow X$. The construction clearly satisfies Items (7.5.1) and (7.5.2).

The canonical decompositions on a torus cover are described as follows.

Proposition 7.6 (Canonical decompositions on torus cover). In the standard Setting 4.1, let $\gamma: Y \rightarrow X$ be a torus cover, $Y=A \times Z$. Then, the Kähler manifold $\left(Y_{\mathrm{reg}}, \omega_{H_{Y}}\right)$ splits as $\left(A, \omega_{H_{A}}\right) \times\left(Z_{\mathrm{reg}}, \omega_{H_{Z}}\right)$, where $\omega_{H_{A}}$ is flat and $\omega_{H_{Z}}$ is Ricci-flat. If $y=(a, z) \in \gamma^{-1}(x)$, the summands in the canonical decompositions of $V_{Y}$ relate to the product structure of $Y$ as follows.

(7.6.1) The summands $V_{Y, 0}$ and $W_{Y, 0}$ both equal $\left.\operatorname{pr}_{1}^{*}(T A)\right|_{y}$.

(7.6.2) The summand $W_{Y, 1} \oplus \cdots \oplus W_{Y, k}$ equals $\left.\operatorname{pr}_{2}^{*}(T Z)\right|_{y}$.

Figure 7.3 on the next page illustrates the canonical decompositions.

7.3.1. Preparation for the proof of Proposition 7.6. Proposition 7.6 relies on a remarkable result of Druel concerning algebraic integrability of foliations, [Dru18]. Druel relates flat subsheaves of $\mathscr{T}_{X}$ to torus factors in suitable covers, but does so only for terminal varieties. We apply Druel's result in the following, slightly indirect manner. 


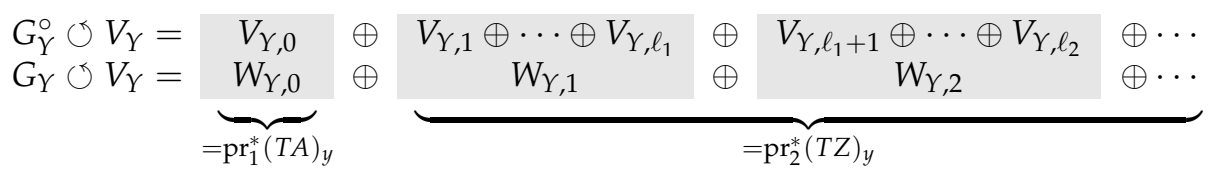

FIGURE 7.3. Canonical decompositions on a torus cover

Lemma 7.7 (Flat summands and augmented irregularity). Let X be a projective klt variety with numerically trivial canonical divisor and assume that there exists a direct sum decomposition $\mathscr{T}_{X}=\mathscr{E} \oplus \mathscr{F}$ where the locally free sheaf $\left.\mathscr{E}\right|_{X_{\mathrm{reg}}}$ is holomorphically flat. Then, $\widetilde{q}(X) \geq \operatorname{rank} \mathscr{E}$.

Proof. -

Step 1: Proof in case where $X$ is terminal. Choose an ample Cartier divisor $H \in$ $\operatorname{Div}(X)$ and recall from Proposition 6.6 or [Gue16, Thm. A] that $\mathscr{T}_{X}$ is $\mathrm{H}$ polystable. The sheaf $\mathscr{F}$ thus decomposes into a direct sum of $H$-stable sheaves, $\mathscr{F}=\mathscr{F}_{1} \oplus \cdots \oplus \mathscr{F}_{m}$. The intersections of $[H]^{n-1}$ with the first Chern class of the $\mathscr{F}_{i}$ clearly vanishes. Moreover, it follows from Flenner's version of the MehtaRamanathan Theorem, [HL10, Thm. 7.1.1], and the Bogomolov inequality, [HL10, Thm. 3.4.1], that $c_{2}\left(\mathscr{F}_{i}\right) \cdot[H]^{n-2} \geq 0$ for all $i$. Renumbering if necessary, we find a number $k$ such that $c_{2}\left(\mathscr{F}_{i}\right) \cdot[H]^{n-2}=0$ if and only if $i \leq k$. Using the assumption that $X$ is terminal, Druel then shows in [Dru18, Cor. 5.8] that

$$
\widetilde{q}(X)=\operatorname{rank}\left(\mathscr{E} \oplus \mathscr{F}_{1} \oplus \cdots \oplus \mathscr{F}_{k}\right) \geq \operatorname{rank} \mathscr{E} .
$$

Step 2: Proof in case where $X$ is canonical and $\mathscr{E}$ is locally free and flat. In this case, consider a terminalisation, that is, a birational crepant morphism $\tau: \widehat{X} \rightarrow X$ where $\widehat{X}$ is terminal and $Q$-factorial. The existence of a terminalisation is shown in [BCHM10, Cor. 1.4.3]. As the pull-back of a flat bundle, $\tau^{*} \mathscr{E}$ is clearly flat. More is true. The extension theorem for differential forms, [GKKP11, Thm. 1.4], gives an injection $\tau^{*} \mathscr{E} \hookrightarrow \mathscr{T}_{\widehat{x}}$ and the polystability result of Guenancia, [Gue16, Thm. A], shows that $\tau^{*} \mathscr{E}$ is in fact a direct summand there. We obtain inequalities

$$
\begin{aligned}
\widetilde{q}(X) & \geq \widetilde{q}(\widehat{X}) & & \text { by Item (2.21.3) of Lemma } 2.21 \\
& \geq \operatorname{rank} \tau^{*} \mathscr{E}=\operatorname{rank} \mathscr{E} & & \text { by Step 1. }
\end{aligned}
$$

Step 3: Proof in general. Let $\alpha: X^{\prime} \rightarrow X$ be a global index-one cover, see Proposition 2.18, which is quasi-étale. The variety $X^{\prime}$ is then canonical with trivial canonical class. Next, let $\beta: Y \rightarrow X^{\prime}$ be a maximally quasi-étale cover, as given by [GKP16c, Thm. 1.5]. The composition $\gamma: Y \rightarrow X$ is then quasi-étale, the variety $Y$ is canonical with trivial canonical class, and the algebraic fundamental groups $\widehat{\pi}_{1}(Y)$ and $\widehat{\pi}_{1}\left(Y_{\text {reg }}\right)$ agree. The extension theorem for flat sheaves, [GKP16c, Thm. 1.14] thus asserts that $\gamma^{[*]} \mathscr{E}$ is locally free and flat. Recalling from Item (2.21.3) of Lemma 2.21 that $\widetilde{q}(X)=\widetilde{q}(Y)$, an application of Step 2 to the direct sum decomposition

$$
\mathscr{T}_{Y}=\gamma^{[*]} \mathscr{E} \oplus \gamma^{[*]} \mathscr{F}
$$

thus finishes the proof of Lemma 7.7 in the general case.

7.3.2. Proof of Proposition 7.6. Assumption (7.5.2) on the augmented irregularity implies that $q(Z)=0$. The ample Cartier divisor $H_{Y} \in \operatorname{Div}(Y)$ is therefore linearly equivalent to a sum $H_{Y} \sim \operatorname{pr}_{1}^{*} H_{A}+\operatorname{pr}_{2} H_{Z}$, where $H_{A}$ and $H_{Z}$ are ample divisors on $A$ and $Z$, respectively. For a proof, choose a point $a \in A$ and consider the fibre $Y_{a}$. The fibre is isomorphic to $Z$, and can be seen as an image of a section $\sigma: Z \rightarrow Y$. 
Set $H_{Z}:=\sigma^{*} H_{Y}$ and $H_{Y}^{\prime}:=H_{Y}-\operatorname{pr}_{2}^{*} H_{Z}$. The restriction of $H_{Y}^{\prime}$ to $Y_{a}$ is linearly trivial. Since $q(Z)=0$, the Picard group of $Z$ is discrete, and the restriction of $H_{Y}^{\prime}$ to other fibres $\left(\left(H_{Y}\right)_{b}\right)_{b \in A}$ is likewise trivial. The push-forward $\left(\operatorname{pr}_{1}\right)_{*} \mathscr{O}_{A}\left(H_{Y}^{\prime}\right)$ is thus invertible. Let $H_{A}$ be an associated Cartier divisor, and observe that $H_{Y}$ is linearly equivalent to $\mathrm{pr}_{1}^{*} H_{A}+\mathrm{pr}_{2} H_{Z}$, as desired.

As a consequence of the decomposition of $H_{Y}$, recall from Proposition 3.7 that the Ricci-flat Kähler manifold $\left(Y_{\text {reg, }}, \omega_{H_{Y}}\right)$ is a product of $\left(A, \omega_{H_{A}}\right)$ and $\left(Z_{\mathrm{reg}}, \omega_{H_{Z}}\right)$. Recall from [Bes87, Sect. 10.35 and Cor. 10.48] that the (restricted) holonomy group then also decomposes. More precisely, writing $y=(a, z)$,

$$
\begin{aligned}
G_{Y}^{\circ} & =\operatorname{Hol}\left(A, g_{H_{A}}\right)_{a}^{\circ} \times \operatorname{Hol}\left(Z_{\mathrm{reg}}, g_{H_{Z}}\right)_{z}^{\circ} \\
G_{Y} & =\operatorname{Hol}\left(A, g_{H_{A}}\right)_{a} \times \operatorname{Hol}\left(Z_{\mathrm{reg}}, g_{H_{Z}}\right)_{z} .
\end{aligned}
$$

By the classical Bochner principle, the elements of a global holomorphic frame for $T A$ are parallel with respect to $g_{H_{A}}$. It follows that $g_{H_{A}}$ is flat and that the holonomy group $\operatorname{Hol}\left(A, g_{H_{A}}\right)_{a}$ is trivial. This implies that $\left.\operatorname{pr}_{1}^{*}(T A)\right|_{y} \subseteq W_{Y, 0}$. On the other hand, Lemma 7.7 asserts that the canonical decomposition of $\mathscr{T}_{Z}$ does not contain a flat summand. Item (6.6.1) of Proposition 6.6 therefore implies that the restricted holonomy of $Z$ has no nonzero fixed points, from which we obtain the remaining inclusion $\left.V_{Y, 0} \subseteq \operatorname{pr}_{1}^{*}(T A)\right|_{y}$. In summary, we have seen that

$$
\left.\left.\operatorname{pr}_{1}^{*}(T A)\right|_{y} \subseteq W_{Y, 0} \subseteq V_{Y, 0} \subseteq \operatorname{pr}_{1}^{*}(T A)\right|_{y} .
$$

Proposition 7.6 is thus shown.

7.4. Proof of Theorem 7.1. The following lemma provides the last missing piece for the proof of Theorem 7.1. Using the classification of restricted holonomy groups found in Section 5, it relates finiteness of $G / G^{\circ}$ to the augmented irregularity of the underlying space. It crucially uses the existence of a "maximally quasi-étale cover", [GKP16c, Thm. 1.5], and hence a global algebro-geometric result.

Lemma 7.8 (Finiteness of connected components). In the standard Setting 4.1, assume that $\widetilde{q}(X)=0$. Then, $G / G^{\circ}$ is finite. In particular, there exists a quasi-étale cover $\gamma: Y \rightarrow X$ where holonomy and restricted holonomy agree, $G_{Y}=G_{Y}^{\circ}$.

Proof. If $G / G^{\circ}$ is finite, then the kernel of the natural surjection $\pi_{1}\left(X_{\text {reg }}\right) \rightarrow G / G^{\circ}$ will yield the desired quasi-étale cover $\gamma$ claimed in the lemma above.

Moreover, by Corollary 4.8 finiteness of $G / G^{\circ}$ follows from finiteness of $G_{Y} / G_{Y}^{\circ}$, where $Y \rightarrow X$ is any quasi-étale cover. Note that every such cover will also have vanishing augmented irregularity. Based on this observation, let us make two reduction steps. First, using the assumption that $\widetilde{q}(X)=0$, Item (7.6.1) of Proposition 7.6 asserts that $V_{0}=\{0\}$. Replacing $X$ by its weak holonomy cover, cf. Proposition 7.3, we may therefore assume without loss of generality that the canonical decompositions of $V$ agree. More precisely, we have $V_{0}=W_{0}=\{0\}$ and $V_{i}=W_{i}$ for all remaining indices $i$, as illustrated in Figure 7.4 on the following page. Second, we can further replace $X$ by a maximally quasi-étale cover, as given by [GKP16c, Thm. 1.5]. This guarantees that any complex representation of $\pi_{1}\left(X_{\text {reg }}\right)$ factors via a representation of $\pi_{1}(X)$ by [GKP16c, Thm. 1.14 and its proof].

To prove that $G / G^{\circ}$ is finite, recall that the holonomy group $G$ is a subgroup of $\mathrm{U}(V)$ and $G^{\circ}$ is its maximal connected subgroup. The group $G$ is therefore contained in the normaliser of $G^{\circ}$ in $\mathrm{U}(V)$. In the special situation at hand, where $G$ and $G^{\circ}$ are both totally decomposed with identical canonical decompositions, 


$$
\begin{array}{rlllllll}
G^{\circ} \circlearrowleft V= & V_{0} & \oplus & V_{1} & \oplus & \cdots & \oplus & V_{m} \\
G \circlearrowleft V= & \underbrace{W_{0}}_{=\{0\}} \oplus W_{1} & \oplus & \cdots & \oplus & W_{k}
\end{array}
$$

FIGURE 7.4. Canonical decompositions in the proof of Lemma 7.4 after simplification.

there is more we can say:

$$
G \subseteq N_{1} \times \cdots \times N_{m} \text { where } N_{i}:=\operatorname{Norm}\left(G_{i}^{\circ} \subseteq \mathrm{U}\left(V_{i}\right)\right) .
$$

The groups $G_{i}^{\circ}$ have been classified in Proposition 5.3, and their normalisers are found by an elementary computation - compare with [Bes87, Prop. 10.114] but observe that we unlike [Bes87] consider normalisers in the unitary group and not in the orthogonal group. Writing $n_{i}:=\operatorname{dim} V_{i}$, either one of the following holds.

- The group $G_{i}^{\circ}$ is isomorphic to $\mathrm{SU}\left(n_{i}\right)$ and $N_{i} / G_{i}^{\circ} \cong \mathrm{U}(1)$.

- The number $n_{i}$ is even, $G_{i}^{\circ} \cong \operatorname{Sp}\left(\frac{n_{i}}{2}\right)$, and $N_{i} / G_{i}^{\circ} \cong \mathrm{U}(1)$. In summary, we see that $G / G^{\circ} \subsetneq \mathrm{U}(1)^{\times m}$ is Abelian.

By the second reduction made at the beginning of the proof, the complex representation $\pi_{1}\left(X_{\text {reg }}\right) \rightarrow G / G^{\circ} \subsetneq \mathrm{U}(1)^{\times m}$ factors via $\pi_{1}(X)$, and hence further via the Abelianisation $H_{1}(X, \mathbb{Z})$. In other words, we obtain a surjection

$$
H_{1}(X, \mathbb{Z}) \rightarrow G / G^{\circ} \text {. }
$$

We claim that $H_{1}(X, \mathbb{Z})$ is finite. Indeed, otherwise $H^{1}(X, \mathbb{Z})$ would be of positive rank. Moreover, the exponential sequence on the complex space $X^{a n}$, [Gun90, proof of Thm. 6 in Chap. K], together with GAGA would yield an embedding $H^{1}(X, \mathbb{Z}) \hookrightarrow H^{1}\left(X, \mathscr{O}_{X}\right)$. But the latter space vanishes owing to $\widetilde{q}(X)=0$, a contradiction. This concludes the proof of Lemma 7.8.

With all preparations in place, the proof of Theorem 7.1 is now easy.

Proof of Theorem 7.1. Let $\gamma$ be a composition of quasi-étale covers,

$$
A \times Z^{\prime} \stackrel{b}{\longrightarrow} A \times Z \frac{a}{\text { torus cover }} X X
$$

where $a$ is a torus cover of $X$, and $b$ is the product of the identity $\operatorname{Id}_{A}$ and the cover of $Z$ constructed in Lemma 7.8. One checks immediately that $\gamma$ is a torus cover, and also satisfies remaining conditions spelled out in Theorem 7.1.

7.5. Proof of Corollary 7.2. Let $\gamma: Y \rightarrow X$ be a holonomy cover. Item (7.2.1) is a consequence of Corollary 4.8 and Lemma 7.8. Item (7.2.2) follows immediately from (7.1.1) and (7.1.3) combined. Item (7.2.3) follows from Lemma 7.7, using the observation that $\mathscr{W}_{i}^{\circ}$ is holomorphically flat if and only if its pull-back $\gamma^{*} \mathscr{W}_{i}^{\circ}$ is holomorphically flat on $A \times Z_{\text {reg. }}$.

7.6. Decomposition of the tangent sheaf. Combining the existence of a holonomy cover with the classification of restricted holonomy, we obtain a holonomy cover whose canonical decomposition of the tangent sheaf admits are particularly precise description.

Proposition 7.9 (Decomposition of the tangent sheaf). In the standard Setting 4.1, there exists a holonomy cover $\gamma: A \times Z \rightarrow X$ such that in addition to properties (1.1.1) (1.1.3), there exists a direct sum decomposition of the tangent sheaf of $Z$,

$$
\mathscr{T}_{Z}=\bigoplus_{i \in I} \mathscr{E}_{i} \oplus \bigoplus_{j \in J} \mathscr{F}_{j}
$$


where the reflexive sheaves $\mathscr{E}_{i}$ (resp. $\left.\mathscr{F}_{j}\right)$ satisfy the following properties.

(7.9.1) The subsheaves $\mathscr{E}_{i} \subseteq \mathscr{T}_{Z}$ (resp. $\mathscr{F}_{j} \subseteq \mathscr{T}_{Z}$ ) are foliations with trivial determinant, of rank $n_{i} \geq 3$ (resp. of even rank $2 m_{j} \geq 2$ ). Moreover, they are strongly stable in the sense of [GKP16b, Def. 7.2].

(7.9.2) On $Z_{\text {reg, }}$ the $\mathscr{E}_{i}$ (resp. $\mathscr{F}_{j}$ ) are locally free and correspond to holomorphic subbundles $E_{i}\left(\right.$ resp. $\left.F_{j}\right)$ of $T Z_{\text {reg }}$ that are parallel with respect to the Levi-Civita connection of $g_{Z}$, the Riemannian metric on $Z_{\text {reg }}$ induced by $\omega_{Z}$. Moreover, their holonomy groups are $\mathrm{SU}\left(n_{i}\right)$ and $\mathrm{Sp}\left(m_{j}\right)$, respectively.

(7.9.3) If $x \in X_{\mathrm{reg}}$ and $(a, z) \in \gamma^{-1}(x)$, then the splitting

$$
T_{x} X \cong T_{(a, z)}(A \times Z)=T_{a} A \oplus \bigoplus_{i \in I} E_{i, z} \oplus \bigoplus_{j \in J} F_{j, z}
$$

corresponds to the decomposition of $T_{x} X$ into irreducible representations under the action of the restricted holonomy group $\mathrm{Hol}\left(X_{\mathrm{reg}}, g_{H}\right)$ at $x$.

Proof. Theorem 7.1 yields a quasi-étale cover $\gamma: A \times Z \rightarrow X$ that satisfies the properties listed in (7.1.1)-(7.1.4). Observe that all these remain true if we replace $Z$ by a further quasi-étale cover. According to Section 6.3.2, we may therefore choose $Z$ such that the summands in the canonical decomposition $\mathscr{T}_{Z}=\mathscr{W}_{0} \oplus \cdots \oplus \mathscr{W}_{k}$ are strongly stable foliations with trivial determinant. By construction, the $\mathscr{W}_{i}$ are locally free on $Z_{\text {reg }}$ and correspond to holomorphic subbundles of $T Z_{\text {reg }}$ that are parallel with respect to the Levi-Civita connection.

We prove Item (7.9.2) next. Corollary 7.2 guarantees that none of the summands $\mathscr{W}_{i}$ is holomorphically flat. Since determinants are trivial, this already guarantees that rank $\mathscr{W}_{i} \geq 2$ for all $i$. More is true. Theorem 7.1 guarantees holonomy and restricted holonomy agree, $G_{Y}^{\circ}=G_{Y}$, and the classification of the restricted holonomy, Proposition 5.3 guarantees that the associated factors of the holonomy group $G=\operatorname{Hol}\left(Z_{\mathrm{reg}}, g_{Z}\right)=G_{1} \times \cdots \times G_{k}$ are isomorphic to either $\operatorname{SU}\left(n_{i}\right)$ or to $\operatorname{Sp}\left(m_{j}\right)$, respectively. Writing $\mathscr{E}_{i}$ for those summands with holonomy $\mathrm{SU}\left(n_{i}\right)$ and $\mathscr{F}_{j}$ for the others, Item (1.2.2) follows.

Item (7.9.1) summarises the results of the two paragraphs above. Item (7.9.3) holds by construction.

\section{Part III. The Bochner principle on singular spaces}

\section{THE BOCHNER PRINCIPLE FOR REFLEXIVE TENSORS AND BUNDLES}

8.1. The Bochner Principle. The goal of this section is to establish the following "Bochner principle", which generalises [Gue16, Thm. C].

Theorem 8.1 (Bochner principle for bundles). In the standard Setting 4.1, given any two numbers $p, q \in \mathbb{N}$, consider the following sheaf of "reflexive tensors",

$$
\mathscr{E}:=\left(\mathscr{T}_{X}^{\otimes p} \otimes\left(\mathscr{T}_{X}^{*}\right)^{\otimes q}\right)^{* *} .
$$

Denote the associated bundle on $X_{\mathrm{reg}}$ by $E$ and recall that the holonomy group $G$ acts on the vector space $E_{x}$ in a canonical manner. Then, the following holds.

(8.1.1) There exists a direct sum decomposition $\mathscr{E}=\mathscr{E}_{1} \oplus \cdots \oplus \mathscr{E}_{k}$ whose summands are stable of slope zero with respect to any polarisation. The induced subbundles $\mathscr{E} \mid X_{\text {reg }}$ of E are parallel with respect to the connection induced by the Chern connection of $\left(T X_{\mathrm{reg}}, h_{H}\right)$. In particular, $\mathscr{E}$ is polystable with respect to any polarisation.

(8.1.2) The fibre map $\mathscr{E} \rightarrow E_{x}$ induces a one-to-one correspondence between arbitrary direct summands of $\mathscr{E}$ and $G$-invariant complex subspaces of $E_{x}$. 
A non-zero section $\sigma$ of $\mathscr{E}$ is the same thing as a trivial subsheaf $\mathscr{O}_{X} \subset \mathscr{E}$. Using semistability arguments, group-theoretic considerations and the Bochner principle just proven, we will show that every such section yields a G-fixed point $\sigma_{x} \in E_{x}$ and vice versa.

Theorem 8.2 (Bochner principle for reflexive tensors). Setting as in Theorem 8.1. Then, the natural evaluation map induces an isomorphism $H^{0}(X, \mathscr{E}) \rightarrow E_{x}^{G}$. In particular, the restriction of every holomorphic tensor to $X_{\mathrm{reg}}$ is parallel.

Remark 8.3. As $\Omega_{\widetilde{X}}^{[p]}$ and Sym ${ }^{[p]} \Omega_{X}^{1}$ are direct summands of $\left(\left(\mathscr{T}_{\widetilde{X}}^{*}\right)^{\otimes p}\right)^{* *}$, the Bochner principle also applies to (symmetric) differential forms.

Theorems 8.1 and 8.2 will be shown in Sections 9 and 10 below. The following corollary, which we promised in Section 6.2, gives an additional description of the summands in the canonical decomposition of $\mathscr{T}_{X}$.

Corollary 8.4 (Summands in the canonical decomposition of $\mathscr{T}_{X}$ ). In the standard Setting 4.1, consider the canonical decomposition of $\mathscr{T}_{X}$, as in (6.4.1). Then, the sheaf $\mathscr{W}_{0}$ is trivial and none of the sheaves $\mathscr{W}_{1}, \ldots, \mathscr{W}_{k}$ and $\mathscr{W}_{1}^{*}, \ldots, \mathscr{W}_{k}^{*}$ admits a section.

Proof. The triviality of $\mathscr{W}_{0}$ has already been established in Item (6.6.3) of Proposition 6.6. Now, let us assume that $\mathscr{W}_{i}$ has a non-zero section $s$ for some $i>0$. By the Bochner principle for reflexive tensors, Theorem $8.2, s$ is parallel hence $s_{x} \neq 0$ and the line $\mathbb{C} \cdot s_{x} \subset W_{i}$ is point-wise fixed by $G$. Irreducibility of $W_{i}$ implies that $\mathbb{C} \cdot s_{x}=W_{i}$ which leads to $W_{i} \subset W_{0}$ and the contradiction follows. The same argument applies to $\mathscr{W}_{i}^{*}$.

8.2. Applications. The Bochner principle allows us to carry over many arguments from the smooth to our singular case. As a sample application, we generalise [Bea83a, Sect. 1, Item (iii) of Cor.] to our setup. See Corollary 11.4 as well as [Pet94, Sect. 3] for related results.

Corollary 8.5 (Detecting finite quotients of Abelian varieties, I). In the standard Setting 4.1, the following inequality holds for all $p \in \mathbb{N}$,

$$
h^{0}\left(X, \Omega_{X}^{[p]}\right) \leq\left(\begin{array}{l}
n \\
p
\end{array}\right) .
$$

If equality holds for one $0<p<n$, then $X$ is of the form $A / \Gamma$ where $A$ is an Abelian variety and $\Gamma$ is a finite group whose action on $A$ is free in codimension one.

Remark 8.6. If $X$ is smooth and equality holds in (8.5.1) for some $0<p<n$, Beauville proves that $X$ is an Abelian variety already, so that no quasi-étale cover is needed. However, this is false in general if $X$ is singular. Indeed, take $X=$ $A /\langle \pm 1\rangle$, the quotient of an Abelian fourfold $A$ by the natural involution $x \mapsto-x$. As every holomorphic two-form on $A$ is preserved by the involution, the maximal value $h^{0}\left(X, \Omega_{X}^{[2]}\right)=6$ is attained, yet $X$ is not Abelian.

Proof of Corollary 8.5. Inequality (8.5.1) follows immediately from the Bochner principle for $\mathscr{E}:=\left(\mathscr{T}_{X}^{*}\right)^{[p]}$. Now assume that there exists one index $0<p<n$ for which equality holds. We employ the decomposition $V=V_{0} \oplus V_{1} \oplus \cdots \oplus V_{m}$ induced by the restricted holonomy group $G^{\circ}=G_{1}^{\circ} \times \cdots \times G_{m}^{\circ}$, as discussed in Construction 5.1. The Bochner principle gives an embedding $H^{0}\left(X, \Omega_{X}^{[p]}\right) \hookrightarrow$ $\left(\wedge^{p} V^{*}\right)^{G^{\circ}}$, and therefore shows that

$$
h^{0}\left(X, \Omega_{X}^{[p]}\right) \leq \operatorname{dim}\left(\wedge^{p} V^{*}\right)^{G^{\circ}} \leq \sum_{\substack{k_{0}, \ldots, k_{m} \in \mathbb{N} \\ \sum k_{j}=p}}\left(\operatorname{dim} \wedge^{k_{0}} V_{0}^{*}\right) \cdot \prod_{i=1}^{m} \operatorname{dim}\left(\wedge^{k_{i}} V_{i}^{*}\right)^{G_{i}^{\circ}}
$$


If $m>0$, then the factors on the right are obviously estimated as follows,

$$
\operatorname{dim}\left(\wedge^{k_{i}} V_{i}^{*}\right)^{G_{i}^{\circ}} \leq \operatorname{dim} \wedge^{k_{i}} V_{i}^{*} \quad \text { for all } i>0 \text { and all } k_{i}
$$

To conclude, by using Vandermonde's Identity for binomial coefficients observe that equality in (8.5.1) can only happen if equality happens in (8.7.1) for all $i>0$ and all $k_{i}$. But then the groups $G_{i}^{\circ}$ would necessarily be trivial, given Proposition 5.3. The assumption that $m>0$ is thus absurd. Equality in (8.5.1) therefore implies that $V_{0}=V$, and Corollary 8.5 follows from Theorem 7.1.

Corollary 8.8 (Restricted holonomy is independent of choice of polarisation). In the standard Setting 4.1, the isomorphism class of the restricted holonomy group $\operatorname{Hol}\left(X_{\mathrm{reg}}, g_{H}\right)^{\circ}$ does not depend on the ample polarisation $H$.

Proof. Assume we are given two ample Cartier divisors $H, H^{\prime}$ on $X$. Theorem 7.1 allows to pass to a holonomy cover. Since this does not affect the restricted holonomy, we may assume without loss of generality that the holonomy groups $G_{H}$ and $G_{H^{\prime}}$ are connected already on $X$. The classification of restricted holonomy, Proposition 5.3, thus equips these groups with a product structure. More precisely, we obtain two canonical decompositions of the tangent sheaf into stable subsheaves,

$$
\mathscr{T}_{X} \cong \mathscr{O}_{X}^{\oplus r} \oplus \bigoplus_{i} \mathscr{E}_{i} \cong \mathscr{O}_{X}^{\oplus r^{\prime}} \oplus \bigoplus_{j} \mathscr{E}_{j}^{\prime},
$$

where the respective holonomy groups act trivially on the trivial parts and are given by the products $G_{H}=\prod G_{i}$ and $G_{H^{\prime}}=\prod G_{j}^{\prime}$, where $G_{i}$ (resp. $G_{j}^{\prime}$ ) is isomorphic either to the the special unitary group or the unitary symplectic group in dimension rank $\mathscr{E}_{i} \geq 2$ (resp. $\operatorname{rank} \mathscr{E}_{j}^{\prime} \geq 2$ ). Clearly, $r=r^{\prime}$, and the remaining stable factors have to be pairwise isomorphic. Up to renumbering, one can assume that $\mathscr{E}_{i} \cong \mathscr{E}_{i}^{\prime}$ and therefore

$$
\bigoplus_{p} H^{0}\left(X, \wedge^{[p]} \mathscr{E}_{i}\right) \cong \bigoplus_{p} H^{0}\left(X, \wedge^{[p]} \mathscr{E}_{i}^{\prime}\right) .
$$

The conclusion then follows from Bochner principle for reflexive tensors, Theorem 8.2, once we observe that $G_{i}$ is special unitary if and only if

$$
h^{0}\left(X, \wedge^{[p]} \mathscr{E}_{i}\right)= \begin{cases}1 & \text { if } p=0 \text { or } p=\operatorname{rank} \mathscr{E}_{i} \\ 0 & \text { otherwise. }\end{cases}
$$

and that $G_{i}$ is unitary symplectic if and only if

$$
h^{0}\left(X, \wedge^{[p]} \mathscr{E}_{i}\right)= \begin{cases}1 & \text { if } 0 \leq p \leq \operatorname{rank} \mathscr{E}_{i} \text { and } p \text { is even } \\ 0 & \text { otherwise. }\end{cases}
$$

\section{PROOF OF THEOREM 8.1 (“BOCHNER PRINCIPLE FOR BUNDLES”)}

We maintain notation and assumptions of Theorem 8.1 in this section. The proof is similar to the one of [Gue16, Thm. A], which deals with the case $\mathscr{E}=\mathscr{T}_{X}$.

Step 1. Setup. Let $\pi: \widetilde{X} \rightarrow X$ be a strong log-resolution of singularities of $X$. Throughout the proof, we discuss the following objects on $\widetilde{X}$.

Discrepancy divisors. Write the standard $Q$-linear equivalence

$$
K_{\widetilde{X}}+\sum a_{i} \cdot D_{i} \sim_{\mathbb{Q}} \pi^{*} K_{X}
$$

where $D=\sum a_{i} D_{i}$ is a $\pi$-exceptional $Q$-divisor with simple normal crossing support and coefficients $a_{i} \in(-\infty, 1) \cap \mathbb{Q}$. Choose sections $s_{i} \in H^{0}\left(\widetilde{X}, \mathscr{O}_{\widetilde{X}}\left(D_{i}\right)\right)$ that vanish precisely on $D_{i}$. 
Metrics and currents. We fix a Kähler reference metric $\omega_{0}$ on $\widetilde{X}$, but we will also consider the pull-back $\omega_{\widetilde{H}}:=\pi^{*} \omega_{H}$. Recall from Remark 3.2 that "pull-back" is meaningful in our context. Set $\widetilde{H}:=\pi^{*} H \in \operatorname{Div}(\widetilde{X})$ and observe that $\operatorname{Ric} \omega_{\widetilde{H}}$ equals the current of integration of $D, \operatorname{Ric} \omega_{\widetilde{H}}=[D]$.

Equip the line bundles $\mathscr{O}_{\widetilde{X}}\left(D_{i}\right)$ with Hermitian metrics $|\cdot|_{i}$, and write $\Theta_{i} \in$ $\mathcal{A}_{\mathbb{R}}^{1,1}(\widetilde{X})$ for the associated curvature form.

Bundles. In analogy to the definition of $E$, consider the bundle $\widetilde{E}:=T \widetilde{X}^{\otimes p} \otimes$ $\left(T^{*} \widetilde{X}\right)^{\otimes q}$, as well as the associated locally free sheaf $\widetilde{\mathscr{E}}:=\mathscr{T}_{\widetilde{X}}^{\otimes p} \otimes\left(\mathscr{T}_{\widetilde{X}}^{*}\right)^{\otimes q}$. We aim to establish polystability of $E$ by studying $\widetilde{E}$.

Step 2. Construction of smooth metrics. As in [Gue16, p. 518], we aim to construct sequences of smooth metrics converging to $\omega_{\widetilde{H}}$.

Construction 9.1 (Construction of smooth metrics $\omega_{t, \varepsilon}$ ). Given a pair of numbers $\varepsilon, t \in \mathbb{R}^{+}$, consider the following form,

$$
\theta_{\varepsilon}:=\sum a_{i}(\underbrace{\Theta_{i}+d d^{c} \log \left(\left|s_{i}\right|_{i}^{2}+\varepsilon^{2}\right)}_{=: \theta_{i, \varepsilon}}) \text { in } \mathcal{A}_{\mathbb{R}}^{1,1}(\widetilde{X}) .
$$

We view $\theta_{\varepsilon}$ as a regularisation of the current of integration $[D]$. The discrepancy formula (9.0.1) implies that $\left\{\theta_{\varepsilon}\right\}=c_{1}(\widetilde{X})$ in $H^{1,1}(\widetilde{X})$. We let $\omega_{t, \varepsilon}$ be the unique Kähler metric on $\widetilde{X}$ whose class equals

$$
\left\{\omega_{t, \varepsilon}\right\}=c_{1}(\widetilde{H})+t \cdot\left\{\omega_{0}\right\} \in H^{1,1}(\widetilde{X}),
$$

and that solves the equation

$$
\operatorname{Ric} \omega_{t, \varepsilon}=\theta_{\varepsilon} .
$$

For existence and uniqueness, see [Yau78].

Remark 9.2 (Convergence of $\omega_{t, \varepsilon}$ for $(\varepsilon, t) \rightarrow(0,0)$ ). It follows from the proof of [EGZ09, Thm. 3.5] and from the uniqueness result [GZ07, Thm. 3.3] that the smooth Kähler forms $\omega_{t, \varepsilon}$ converge on $\widetilde{X} \backslash \operatorname{supp} D$ to the singular Kähler-Einstein metric $\omega_{\widetilde{H}}$. More precisely, we have

$$
\lim _{(\varepsilon, t) \rightarrow(0,0)} \omega_{t, \varepsilon}=\omega_{\widetilde{H}}
$$

on $\widetilde{X} \backslash \operatorname{supp} D$ in the $\mathcal{C}_{\text {loc }}^{\infty}$-topology.

Remark 9.3. Let $\nabla_{i}^{\prime}$ be the $(1,0)$-part of the Chern connection of $\left(\mathscr{O}_{\widetilde{X}}\left(D_{i}\right),|\cdot|_{i}\right)$. A direct computation shows that the form $\theta_{i, \varepsilon} \in \mathcal{A}_{\mathbb{R}}^{1,1}(\widetilde{X})$ decomposes as follows,

$$
\theta_{i, \varepsilon}=\underbrace{\frac{\varepsilon^{2} \cdot\left|\nabla_{i}^{\prime} s_{i}\right|_{i}^{2}}{\left(\left|s_{i}\right|_{i}^{2}+\varepsilon^{2}\right)^{2}}}_{=: \beta_{i, \varepsilon}}+\underbrace{\frac{\varepsilon^{2} \cdot \Theta_{i}}{\left|s_{i}\right|_{i}^{2}+\varepsilon^{2}}}_{=: \gamma_{i, \varepsilon}} .
$$

We refer to [CGP13, beginning of Sect. 3] for an analogous computation. The summands $\beta_{i, \varepsilon}$ and $\gamma_{i, \varepsilon}$ are smooth forms in $\mathcal{A}_{\mathbb{R}}^{1,1}(\widetilde{X})$.

Notation 9.4 (Hermitian metrics and curvature on $T \widetilde{X}$ ). We endow $T \widetilde{X}$ with the Kähler form $\omega_{t, \varepsilon}$, so $T \widetilde{X}$ and more generally $\widetilde{E}$ can be equipped with the structure of a holomorphic Hermitian vector bundles. We denote by $\widetilde{h}_{t, \varepsilon}$ the Hermitian metric on $\widetilde{E}$ induced by $\omega_{t, \varepsilon}$ and write $\Theta_{\widetilde{h}}(\widetilde{E}) \in \mathcal{A}^{1,1}(\widetilde{X}$, End $(\widetilde{E}))$ for its Chern curvature. The form $i \Theta_{\widetilde{h}_{t, \varepsilon}}(\widetilde{E})$ is a real $(1,1)$-form with values in the Hermitian endomorphisms of $\left(E, \widetilde{h}_{t, \varepsilon}\right)$. 
In the course of the proof we will need the following result, which is proved in [Gue16, Lem. 3.7]. In our context, there is a much simpler proof though, which we give below for the convenience of the reader.

Claim 9.5. For every fixed $t>0$, and every index $i$ we have

$$
0=\lim _{\varepsilon \rightarrow 0} \int_{\widetilde{X}} \frac{\varepsilon^{2}}{\left|s_{i}\right|_{i}^{2}+\varepsilon^{2}} \cdot \omega_{0} \wedge \omega_{t, \varepsilon}^{n-1}
$$

Proof. As the total mass of $\omega_{0} \wedge \omega_{t, \varepsilon}^{n-1}$ is independent of $\varepsilon$, it is enough to prove that $\int_{\left\{\left|s_{i}\right|^{2}<\varepsilon\right\}} \omega_{0} \wedge \omega_{t, \varepsilon}^{n-1}$ converges to 0 as $\varepsilon$ approaches zero. The important observation is that the potentials $\varphi_{t, \varepsilon}$ of $\omega_{t, \varepsilon}$ are uniformly bounded in $\varepsilon$, when $t$ is fixed. This is a consequence of Kołodziej's $L^{p}$-estimate, cf. [Koł98, proof of Thm. 2.4.2, Ex. 2], because $\omega_{t, \varepsilon}^{n}$ has a density $f_{\mathcal{E}}$ with respect to a volume form satisfying $\left\|f_{\varepsilon}\right\|_{L^{p}} \leq C$ where $C$ is independent of $\varepsilon$.

Next, one can introduce a family of cut-off functions $\left(\chi_{\varepsilon}\right)_{\varepsilon>0}$ for the divisor $D_{i}$ as in [CGP13, Sect. 9]. These functions satisfy the important property that their complex Hessian, $d d^{c} \chi_{\varepsilon}$, is uniformly dominated by a metric $\omega_{P}$ with Poincaré type along $D_{i}$. Then, one can perform successive integrations by parts in a similar way as in the proof of Chern-Levine-Nirenberg inequality and see that there exists a uniform constant $C>0$ such that $\int_{\left\{\left|s_{i}\right|^{2}<\varepsilon\right\}} \omega_{0} \wedge \omega_{t, \varepsilon}^{n-1} \leq C \int_{\left\{\left|s_{i}\right|^{2}<\varepsilon\right\}} \omega_{P}^{n}$. The constant incorporates the sup-norms of the various potentials above. Claim 9.5 follows at once from the finiteness of the volume of the Poincare type metric $\omega_{P}$.

Step 3. Computing slopes using $\omega_{t, \varepsilon}$. Given a saturated subsheaf $\mathscr{F} \subseteq \mathscr{E}$, we aim to lift $\mathscr{F}$ to a subsheaf of $\widetilde{\mathscr{F}} \subseteq \widetilde{\mathscr{E}}$, and to compute the slope of $\widetilde{\mathscr{F}}$ with respect to the Kähler metrics $\omega_{t, \varepsilon}$.

Setup and Notation 9.6. Assume we are given a saturated subsheaf $\mathscr{F} \subseteq \mathscr{E}$, which will automatically be reflexive. We write $\widetilde{\mathscr{F}} \subseteq \widetilde{\mathscr{E}}$ for the unique saturated subsheaf that agrees with $\mathscr{F}$ wherever the resolution morphism $\pi$ is isomorphic. We denote the singularity set of $\widetilde{\mathscr{F}} \subseteq \widetilde{\mathscr{E}}$ by $W \subset \widetilde{X}$. This is the minimal closed subset of $\widetilde{X}$ outside which $\widetilde{F} \subseteq \widetilde{E}$ corresponds to a subbundle, which we denote as $\widetilde{F} \subseteq$ $\left.\widetilde{E}\right|_{\widetilde{X} \backslash W}$. Recall that $W$ has codimension at least two. The restriction of $\widetilde{h}_{t, \varepsilon}$ endows $\widetilde{F}$ with a Hermitian structure. Write

$$
\Theta_{\widetilde{h}_{t, \varepsilon}}(\widetilde{F}) \in \mathcal{A}^{1,1}(\widetilde{X} \backslash W, \operatorname{End}(\widetilde{F}))
$$

for its Chern curvature.

Claim 9.7. Setup and notation as in 9.6. Then, the following inequality holds.

$$
n \cdot c_{1}(\widetilde{\mathscr{F}}) \cdot\left\{\omega_{t, \varepsilon}\right\}^{n-1} \leq \int_{\widetilde{X} \backslash W} \operatorname{tr}_{\operatorname{End}}\left(\operatorname{pr}_{\widetilde{F}}\left(\left.\operatorname{tr}_{\omega_{t, \varepsilon}} i \Theta_{\widetilde{h}}(\widetilde{E})\right|_{\widetilde{F}}\right)\right) \cdot \omega_{t, \varepsilon}^{n} \cdot
$$

We refer to the right hand side of (9.7.1) as the error term.

Explanation 9.8. In Equation (9.7.1), $\operatorname{pr}_{\widetilde{F}}$ is the orthogonal projection $\widetilde{E} \rightarrow \widetilde{F}$. The symbol $\operatorname{tr}_{\omega_{t, \varepsilon}}$ denotes the trace relatively to the Kähler metric $\omega_{t, \varepsilon}$. Given a bundle $G$ and a $G$-valued form $\alpha \in \mathcal{A}^{1,1}(G)$, recall that $\operatorname{tr}_{\omega_{t, \varepsilon}} \alpha$ is the unique section of $G$ such that $\left(\operatorname{tr}_{\omega_{t, \varepsilon}} \alpha\right) \otimes \omega_{t, \varepsilon}^{n}=n \cdot \alpha \wedge \omega_{t, \varepsilon}^{n-1}$. The object $\left.\operatorname{tr}_{\omega_{t, \varepsilon}} i \Theta_{\widetilde{h} t, \varepsilon}(\widetilde{E})\right|_{\widetilde{F}}$ in (9.7.1) is therefore a section of $\operatorname{Hom}(\widetilde{F}, \widetilde{E})$.

Proof of Claim 9.7. We aim to relate the curvature of the subbundle $\left(\widetilde{F}, \widetilde{h}_{t, \varepsilon}\right)$ to the one of $\left(\widetilde{E}, \widetilde{h}_{t, \varepsilon}\right)$. Classically, this is done by introducing the second fundamental 
form $\rho_{t, \varepsilon} \in \mathcal{A}^{1,0}\left(\widetilde{X} \backslash W, \operatorname{Hom}\left(\widetilde{F}, \widetilde{F}^{\perp}\right)\right)$, cf. [Dem12, Sect. V.14], which satisfies the relation

$$
\Theta_{\widetilde{h}_{t, \varepsilon}}(\widetilde{F})=\operatorname{pr}_{\widetilde{F}}\left(\left.\Theta_{\widetilde{h}_{t, \varepsilon}}(\widetilde{E})\right|_{\widetilde{F}}\right)+\rho_{t, \varepsilon}^{*} \wedge \rho_{t, \varepsilon} \quad \text { in } \mathcal{A}^{1,1}(\widetilde{X} \backslash W, \operatorname{End}(\widetilde{F})) .
$$

Multiplying by $i$, taking the trace (as endomorphism) and wedging with $\omega_{t, \varepsilon}^{n-1}$ -and observing that the operations of taking the metric trace and taking the endomorphism trace commute- we obtain the following identity,

$$
\begin{aligned}
c_{1}\left(\widetilde{F}, \widetilde{h}_{t, \varepsilon}\right) \wedge \omega_{t, \varepsilon}^{n-1}=\operatorname{tr}_{\text {End }} & \left(\operatorname{pr}_{\widetilde{F}}\left(\left.\operatorname{tr}_{\omega_{t, \varepsilon}} \Theta_{\widetilde{h}_{t, \varepsilon}}(\widetilde{E})\right|_{\widetilde{F}}\right)\right) \cdot \frac{\omega_{t, \varepsilon}^{n}}{n} \\
& +\underbrace{\operatorname{tr}_{\text {End }}\left(i \rho_{t, \varepsilon}^{*} \wedge \rho_{t, \varepsilon} \wedge \omega_{t, \varepsilon}^{n-1}\right)}_{\text {seminegative }} \text { in } \mathcal{A}^{n, n}(\widetilde{X} \backslash W) .
\end{aligned}
$$

To make use of (9.9.1), recall the following identity, which follows for instance from [Kob87, Eq. $\left.{ }^{* *}\right)$ on p. 181$]^{3}$,

$$
\int_{\widetilde{X} \backslash W} c_{1}\left(\widetilde{F}, \widetilde{h}_{t, \varepsilon}\right) \wedge \omega_{t, \varepsilon}^{n-1}=c_{1}(\widetilde{\mathscr{F}}) \cdot\left\{\omega_{t, \varepsilon}\right\}^{n-1} .
$$

Indeed, Inequality (9.7.1) follows by integrating (9.9.1) over $\widetilde{X} \backslash W$ with the help of (9.9.2). Claim 9.7 follows.

Step 4. Analysis of the error term. The aim of the current step is to state the following claim.

Claim 9.10. Setup and notation as in 9.6. Then, the error term converges to zero as $t, \varepsilon \rightarrow 0$. More precisely.

$$
\lim _{(\varepsilon, t) \rightarrow(0,0)} \int_{\widetilde{X} \backslash W} \operatorname{tr}_{\text {End }}\left(\operatorname{pr}_{\widetilde{F}}\left(\left.\operatorname{tr}_{\omega_{t, \varepsilon}} i \Theta_{\widetilde{h}_{t, \varepsilon}}(\widetilde{E})\right|_{\widetilde{F}}\right)\right) \cdot \omega_{t, \varepsilon}^{n}=0 .
$$

Before proving Claim 9.10 in Step 7 below, we need to introduce notation and establish a number of auxiliary results.

Notation 9.11. If $V$ is a complex vector space of dimension $n$ and $f \in \operatorname{End}(V)$, we denote by $f^{\bowtie p}$ the endomorphism of $V^{\otimes p}$ defined on pure tensors by

$$
f^{\bowtie p}\left(v_{1} \otimes \cdots \otimes v_{p}\right):=\sum_{i=1}^{p} v_{1} \otimes \cdots \otimes v_{i-1} \otimes f\left(v_{i}\right) \otimes v_{i+1} \otimes \cdots \otimes v_{p}
$$

Observation 9.12. In the setting of Notation 9.11, one has $\operatorname{tr}\left(f^{\boxplus p}\right)=p \cdot n^{p-1} \cdot \operatorname{tr}(f)$. If $V$ has an Hermitian structure and if $f$ is Hermitian semipositive, then so is $f^{\bowtie p}$.

Step 5: Analysis of $\operatorname{tr}_{\omega_{t, \varepsilon}} \Theta_{\widetilde{h}_{t, \varepsilon}}(\widetilde{E})$. One fundamental object that appears in the error term is $\operatorname{tr}_{\omega_{t, \varepsilon}} i \Theta_{\widetilde{h}_{t, \varepsilon}}(\widetilde{E})$, which is a Hermitian endomorphism of the bundle $\widetilde{E}$. The following claim relates it to the Ricci curvature of $\omega_{t, \varepsilon}$. Its formulation uses the operator " $\sharp t, \varepsilon$ ". We briefly recall the definition.

Construction and Notation 9.13. Using the Kähler metrics $\omega_{t, \varepsilon}$, one constructs from any $(0,1)$-form $\eta$ a $(1,0)$-vector field $\sharp_{t, \varepsilon} \eta$, requiring that the relation

$$
\eta(\bar{\zeta}):=g_{t, \varepsilon}(i \sharp t, \varepsilon \eta, \zeta)
$$

holds for any vector field $\zeta$ of type $(1,0)$, where $g_{t, \varepsilon}$ is the Hermitian metric on $T^{1,0} \widetilde{X}$ associated with the Kähler form $\omega_{t, \varepsilon}$. Next, one extends the operator $\sharp_{t, \varepsilon}$ to vector-valued forms. In particular, if $\alpha \in \mathcal{A}^{1,1}(\widetilde{X})$, one can see $\alpha$ as a $(0,1)$-form

\footnotetext{
${ }^{3}$ see also [Gue16, Prop. 3.8, Case 1]
} 
with values in $\left(T^{1,0} \widetilde{X}\right)^{*}$ and define $\sharp_{t, \varepsilon} \alpha \in \operatorname{End}(T \widetilde{X})$ as follows: in local coordinates, let us write $\omega_{t, \varepsilon}=i \sum_{j, k} g_{j \bar{k}} \cdot d z_{j} \wedge d \bar{z}_{k}$. Let $\left(g^{j j \bar{k}}\right)$ be the inverse of $\left(g_{j \bar{k}}\right)$ let $\alpha=i \sum_{j, k} \alpha_{j \bar{k}} \cdot d z_{j} \wedge d \bar{z}_{k}$ be a $(1,1)$-form. Then,

$$
\sharp_{t, \varepsilon} \alpha=\sum_{j, k, \ell} \alpha_{j \bar{k}} g^{\ell \bar{k}} \cdot d z_{j} \otimes \frac{\partial}{\partial z_{\ell}} .
$$

It is easy to check the formula

$$
\operatorname{tr}_{\text {End } \sharp t, \varepsilon} \alpha=\operatorname{tr}_{\omega_{t, \varepsilon}} \alpha .
$$

Finally, the endomorphism $\sharp_{t, \varepsilon} \alpha$ is Hermitian (resp. Hermitian semipositive) with respect to $g_{t, \varepsilon}$ if $\alpha$ is real (resp. semipositive).

Claim 9.14. Setup and notation as in 9.6. Then,

$$
\operatorname{tr}_{\omega_{t, \varepsilon}} i \Theta_{\widetilde{h}_{t, \varepsilon}}(\widetilde{E})=\left(\sharp_{t, \varepsilon} \theta_{\varepsilon}\right)^{\otimes p} \otimes \operatorname{Id}_{T^{*} \widetilde{X}^{\otimes q}}-\operatorname{Id}_{T \widetilde{X}^{\otimes p}} \otimes{\overline{\left(\sharp_{t, \varepsilon} \theta_{\varepsilon}\right)}}^{\otimes q} \quad \text { in } \operatorname{End}(\widetilde{E}) .
$$

Proof of Claim 9.14. Following the standard computations of [Gue16, p. 524], one obtains the following identities.

$$
\begin{aligned}
& n \cdot i \Theta_{\widetilde{h}_{t, \varepsilon}}\left(T \widetilde{X}^{\otimes p}\right) \wedge \omega_{t, \varepsilon}^{n-1}=\left(\sharp_{t, \varepsilon} \operatorname{Ric} \omega_{t, \varepsilon}\right)^{\otimes p} \cdot \omega_{t, \varepsilon}^{n} \quad \text { in } \mathcal{A}^{n, n}\left(\widetilde{X}, \operatorname{End}\left(T \widetilde{X}^{\otimes p}\right)\right) \\
& n \cdot i \Theta_{\widetilde{h}_{t, \varepsilon}}\left(\left(T^{*} \widetilde{X}\right)^{\otimes q}\right) \wedge \omega_{t, \varepsilon}^{n-1}=-{\left.\overline{(\sharp t, \varepsilon} \operatorname{Ric} \omega_{t, \varepsilon}\right)}^{\otimes q} \cdot \omega_{t, \varepsilon}^{n} \quad \text { in } \mathcal{A}^{n, n}\left(\widetilde{X}, \operatorname{End}\left(T^{*} \widetilde{X}^{\otimes q}\right)\right) \text {. }
\end{aligned}
$$

In summary, we deduce the following identity in in $\mathcal{A}^{n, n}(\widetilde{X}, \operatorname{End}(\widetilde{E}))$,

$$
\begin{aligned}
& n \cdot i \Theta_{\widetilde{h}_{t, \varepsilon}}(\widetilde{E}) \wedge \omega_{t, \varepsilon}^{n-1}= \\
& \left.\left(\left(\sharp_{t, \varepsilon} \operatorname{Ric} \omega_{t, \varepsilon}\right)^{\otimes p} \otimes \operatorname{Id}_{T^{*} \widetilde{X}^{\otimes q}}-\operatorname{Id}_{T \widetilde{X}^{\otimes p}} \otimes \overline{\left(\sharp_{t, \varepsilon} \operatorname{Ric} \omega_{t, \varepsilon}\right)}\right)^{\otimes q}\right) \cdot \omega_{t, \varepsilon}^{n} .
\end{aligned}
$$

Equation (9.1.3) and the definition $\operatorname{tr}_{\omega_{t, \varepsilon}}$, cf. Explanation 9.8, thus imply (9.14.1). This finishes the proof of Claim 9.14.

Step 6: Convergence of integrals. Claim 9.14 reduces the study of the error term to an analysis of the forms $\theta_{\varepsilon}$. We have seen in Step 2 that $\theta_{\varepsilon}$ decomposes as $\theta_{\varepsilon}=$ $\sum_{i} a_{i} \theta_{i, \varepsilon}=\sum_{i} a_{i}\left(\beta_{i, \varepsilon}+\gamma_{i, \varepsilon}\right)$. The present step analyses the contributions to the error term that come from the $\gamma_{i, \varepsilon}$ and $\beta_{i, \varepsilon}$, respectively.

Claim 9.15. Setup and notation as in 9.6. Given any positive number $t$ and any index $i$, the following integrals converge to zero,

$$
\begin{aligned}
& 0=\lim _{\varepsilon \rightarrow 0} \int_{\widetilde{X} \backslash W} \operatorname{tr}_{\text {End }}\left(\operatorname{pr}_{\widetilde{F}}\left(\left.\left(\sharp_{t, \varepsilon} \gamma_{i, \varepsilon}\right)^{\otimes p} \otimes \operatorname{Id}_{T^{*} \widetilde{X} \otimes q}\right|_{\widetilde{F}}\right)\right) \cdot \omega_{t, \varepsilon}^{n} \\
& 0=\lim _{\varepsilon \rightarrow 0} \int_{\widetilde{X} \backslash W} \operatorname{tr}_{\text {End }}\left(\operatorname{pr}_{\widetilde{F}}\left(\left.\operatorname{Id}_{T_{\widetilde{X}}^{\otimes p}} \otimes \overline{\left(\sharp_{t, \varepsilon} \gamma_{i, \varepsilon}\right)}{ }^{\otimes q}\right|_{\widetilde{F}}\right)\right) \cdot \omega_{t, \varepsilon}^{n}
\end{aligned}
$$

Proof of Claim 9.15. Using the special form of $\gamma_{i, \varepsilon}$ found in Remark 9.3, there exists a constant $C \in \mathbb{R}^{+}$such that

$$
\pm \gamma_{i, \varepsilon} \leq \frac{C \cdot \varepsilon^{2}}{\left|s_{i}\right|_{i}^{2}+\varepsilon^{2}} \cdot \omega_{0} \quad \text { in } \mathscr{A}_{\mathbb{R}}^{1,1}(\widetilde{X}), \text { for all } \varepsilon \in \mathbb{R}^{+}
$$


The operations $\sharp_{t, \varepsilon} \bullet, \mathbf{\boldsymbol { 0 }}, \bullet \otimes p$ and $\bullet \otimes$ Id preserve (semi)positivity, cf. Observation 9.12 and Notation 9.13. The following inequalities of Hermitian endomorphisms of $\left(\widetilde{E}, \widetilde{h}_{t, \varepsilon}\right)$ will thus again hold for all $\varepsilon \in \mathbb{R}^{+}$,

$$
\begin{aligned}
& \pm\left(\sharp_{t, \varepsilon} \gamma_{i, \varepsilon}\right)^{\otimes p} \otimes \operatorname{Id}_{T^{*} \widetilde{X}^{\otimes q}} \leq \frac{C \cdot \varepsilon^{2}}{\left|s_{i}\right|_{i}^{2}+\varepsilon^{2}} \cdot\left(\sharp_{t, \varepsilon} \omega_{0}\right)^{\otimes p} \otimes \operatorname{Id}_{T^{*} \widetilde{X}^{\otimes q}} \\
& \pm \operatorname{Id}_{T \widetilde{X}^{\otimes p}} \otimes{\left.\overline{(\sharp t, \varepsilon} \gamma_{i, \varepsilon}\right)}^{\otimes q} \leq \frac{C \cdot \varepsilon^{2}}{\left|s_{i}\right|_{i}^{2}+\varepsilon^{2}} \cdot \operatorname{Id}_{T \tilde{X}^{\otimes p}} \otimes \overline{\left(\sharp t, \varepsilon \omega_{0}\right)}{ }^{\otimes q}
\end{aligned}
$$

As $\sharp t, \varepsilon \omega_{0}$ is a positive endomorphism of $T \widetilde{X}$ whose trace is $\operatorname{tr}_{\omega_{t, \varepsilon}} \omega_{0}$, cf. Eq. (9.13.1), an elementary computation ${ }^{4}$, shows that

$$
\left(\sharp_{t, \varepsilon} \omega_{0}\right)^{\otimes p} \leq p \cdot n^{p-1} \cdot \operatorname{tr}_{\omega_{t, \varepsilon}}\left(\omega_{0}\right) \operatorname{Id}_{T \widetilde{X}^{\otimes p}} \quad \text { in } \operatorname{End}\left(T \widetilde{X}^{\otimes p}, \widetilde{h}_{t, \varepsilon}^{\otimes p}\right) .
$$

Consequently, there exists $C^{\prime} \in \mathbb{R}^{+}$such that the following inequalities of Hermitian endomorphisms of $\left(\widetilde{F}, \widetilde{h}_{t, \varepsilon}\right)$ will hold for all $\varepsilon \in \mathbb{R}^{+}$,

$$
\begin{array}{lll} 
\pm & \operatorname{pr}_{\widetilde{F}}\left(\left.\left(\sharp_{t, \varepsilon} \gamma_{i, \varepsilon}\right)^{\otimes p} \otimes \operatorname{Id}_{T^{*} \widetilde{X} \otimes q}\right|_{\widetilde{F}}\right) & \\
& \leq \frac{C \cdot \varepsilon^{2}}{\left|s_{i}\right|_{i}^{2}+\varepsilon^{2}} \cdot \operatorname{pr}_{\widetilde{F}}\left(\left.\left(\sharp_{t, \varepsilon} \omega_{0}\right)^{\otimes p} \otimes \operatorname{Id}_{T^{*} \widetilde{X} \otimes q}\right|_{\widetilde{F}}\right) & \text { by (9.15.4) } \\
\quad \leq \frac{C^{\prime} \cdot \varepsilon^{2}}{\left|s_{i}\right|_{i}^{2}+\varepsilon^{2}} \cdot \operatorname{tr}_{\omega_{t, \varepsilon}}\left(\omega_{0}\right) \cdot \operatorname{Id}_{\widetilde{F}} . & \text { by (9.15.6) }
\end{array}
$$

Recalling the definition of $\operatorname{tr}_{\omega_{t, \varepsilon}}\left(\omega_{0}\right)$ from Explanation 9.8, we find $C^{\prime \prime} \in \mathbb{R}^{+}$such that the following inequality of real $(n, n)$-forms holds,

$$
\pm \operatorname{tr}_{\operatorname{End}}\left(\operatorname{pr}_{\widetilde{F}}\left(\left.\left(\sharp_{t, \varepsilon} \gamma_{i, \varepsilon}\right)^{\otimes p} \otimes \operatorname{Id}_{T^{*} \widetilde{X} \otimes q}\right|_{\widetilde{F}}\right)\right) \cdot \omega_{t, \varepsilon}^{n} \leq \frac{C^{\prime \prime} \cdot \varepsilon^{2}}{\left|s_{i}\right|^{2}+\varepsilon^{2}} \cdot \omega_{0} \wedge \omega_{t, \varepsilon}^{n-1} .
$$

From Claim 9.5 and Lebesgue's dominated convergence theorem, one deduces the convergence of (9.15.1). Convergence of (9.15.2) follows in a similar fashion, using (9.15.5) in place of (9.15.4). Claim 9.15 follows.

Claim 9.16. Setup and notation as in 9.6. Given any index $i$, the following integrals converge to zero,

$$
\begin{aligned}
& 0=\lim _{(\varepsilon, t) \rightarrow(0,0)} \int_{\widetilde{X} \backslash W} \operatorname{tr}_{\operatorname{End}}\left(\operatorname{pr}_{\widetilde{F}}\left(\left.\left(\sharp t, \varepsilon \beta_{i, \varepsilon}\right)^{\otimes p} \otimes \operatorname{Id}_{T^{*} \widetilde{X} \otimes q}\right|_{\widetilde{F}}\right)\right) \cdot \omega_{t, \varepsilon}^{n} \\
& 0=\lim _{(\varepsilon, t) \rightarrow(0,0)} \int_{\widetilde{X} \backslash W} \operatorname{tr}_{\text {End }}\left(\operatorname{pr}_{\widetilde{F}}\left(\left.\left.\operatorname{Id}_{T \widetilde{X}^{\otimes p}} \otimes \overline{\left(\sharp_{t, \varepsilon} \beta_{i, \varepsilon}\right)}\right|^{\otimes q}\right|_{\widetilde{F}}\right)\right) \cdot \omega_{t, \varepsilon}^{n}
\end{aligned}
$$

Proof of Claim 9.16. Using is special form, we see that $\beta_{i, \varepsilon}$ is a semipositive, real $(1,1)$-form. Using Observation 9.12, and using again that the operations $\sharp_{t, \varepsilon} \bullet \mathbf{e}, \mathbf{\bullet}$, $\bullet \otimes p$ and $\bullet \otimes$ Id preserve semipositivity, we hence obtain the following inequality of real forms in $\mathcal{A}^{n, n}\left(\operatorname{End}(\widetilde{E}), \widetilde{h}_{t, \varepsilon}\right)$,

$$
\left(\left(\sharp t, \varepsilon \beta_{i, \varepsilon}\right)^{\otimes p} \otimes \operatorname{Id}_{T^{*} \widetilde{X}^{\otimes q}}\right) \cdot \omega_{t, \varepsilon}^{n} \leq p n^{p} \cdot \operatorname{Id}_{\widetilde{E}} \cdot \beta_{i, \varepsilon} \wedge \omega_{t, \varepsilon}^{n-1} .
$$

\footnotetext{
${ }^{4}$ Use Observation 9.12 to compute the left hand side.
} 
Therefore, there exists a constant $C \in \mathbb{R}^{+}$such that the following inequalities hold for all values of $t, \varepsilon$,

$$
\begin{array}{rlr}
0 & \leq \int_{\widetilde{X} \backslash W} \operatorname{tr}_{\text {End }}\left(\operatorname{pr}_{\widetilde{F}}\left(\left.\left(\sharp t, \varepsilon \beta_{i, \varepsilon}\right)^{\otimes p} \otimes \operatorname{Id}_{T^{*} \widetilde{X} \otimes q}\right|_{\widetilde{F}}\right)\right) \cdot \omega_{t, \varepsilon}^{n} & \text { semipositivity of } \beta_{i, \varepsilon} \\
& \leq C \cdot \int_{\widetilde{X} \backslash W} \beta_{i, \varepsilon} \wedge \omega_{t, \varepsilon}^{n-1} & \text { Inequality (9.16.3) } \\
& =C \cdot\left(\int_{\widetilde{X}}\left(\beta_{i, \varepsilon}+\gamma_{i, \varepsilon}\right) \wedge \omega_{t, \varepsilon}^{n-1}-\int_{\widetilde{X}} \gamma_{i, \varepsilon} \wedge \omega_{t, \varepsilon}^{n-1}\right) & \\
& =C \cdot(\underbrace{\left\{\theta_{i, \varepsilon}\right\} \cdot\left\{\omega_{t, \varepsilon}\right\}^{n-1}}_{\lim _{t \rightarrow 0}=0, \text { see Eqn. (9.16.4) }}-\underbrace{\int_{\widetilde{X}} \gamma_{t, \varepsilon} \wedge \omega^{n-1}}_{\lim _{\varepsilon \rightarrow 0}=0, \text { by Claim 9.15 }}) &
\end{array}
$$

As for the first term, since $\widetilde{H}=\pi^{*} H$ is orthogonal to $D_{i}$, Equations (9.1.1) and (9.1.2) imply

$$
\left\{\theta_{i, \varepsilon}\right\} \cdot\left\{\omega_{t, \varepsilon}\right\}^{n-1}=t^{n-1}\left(D_{i} \cdot\left\{\omega_{0}\right\}^{n-1}\right), \quad \text { for all } \varepsilon, t \text { and } i .
$$

Consequently, the term converges to 0 when $t$ goes to 0 . Equation (9.16.1) follows. Equation (9.16.2) follows in a similar fashion. This ends the proof of Claim 9.16.

Step 7. Proof of Claim 9.10. Claim 9.10 now follows from Claim 9.14, and the convergence results of Claims 9.15 and 9.16.

Step 8. Proof of Theorem 8.1, Item (8.1.1). We will first prove semistability of $\mathscr{E}$ with respect to $H$. Since the ample divisor $H \in \operatorname{Div}(X)$ was arbitrarily chosen when we fixed the standard Setting 4.1, this will in fact prove semistability of $\mathscr{E}$ with respect to any ample class. To this end, let $\mathscr{F} \subseteq \mathscr{E}$ be any reflexive sheaf. Using Setup and Notation 9.6, we need to show that the number

$$
c_{1}(\mathscr{F}) \cdot[H]^{n-1}=c_{1}(\widetilde{\mathscr{F}}) \cdot[\widetilde{H}]^{n-1} \stackrel{(9.1 .2)}{=} \lim _{(t, \varepsilon) \rightarrow(0,0)} c_{1}(\widetilde{\mathscr{F}}) \cdot\left\{\omega_{t, c}\right\}^{n-1}
$$

is seminegative. But seminegativity of the right hand side follows immediately from Claims 9.7 and 9.10. Semistability follows.

Hence, arguing by induction, to prove the existence of a parallel decomposition $\mathscr{E}=\mathscr{E}_{1} \oplus \cdots \oplus \mathscr{E}_{k}$ whose summands are stable of slope zero with respect to any polarisation, it suffices to show the following claim.

Claim 9.17. Any saturated subsheaf $\mathscr{F} \subseteq \mathscr{E}$ of slope $\mu_{H}(\mathscr{F})=0$ is a direct summand and the associated subbundle $F:=\left.\mathscr{F}\right|_{X_{\text {reg }}}$ is parallel with respect to the connection on $\mathscr{E}_{\mid X_{\text {reg }}}$ induced by the Chern connection of $\left(T X_{\text {reg }}, h_{H}\right)$.

Proof of Claim 9.17. Assume that one such $\mathscr{F}$ is given. Both sides of the Equation (9.16.5) are then zero. Recall from Construction 9.1 that $\omega_{t, \varepsilon}$ converges to $\omega_{\widetilde{H}}$ in the $\mathcal{C}_{\text {loc }}^{\infty}(\widetilde{X} \backslash D)$-topology. Therefore, the second fundamental form $\rho_{t, \varepsilon}$ converges locally smoothly on $\widetilde{X} \backslash(D \cup W)$ to a smooth form $\rho_{\widetilde{H}}$. Moreover, we get from (9.9.1) that

$$
\liminf _{(t, \varepsilon) \rightarrow(0,0)} \int_{\widetilde{X} \backslash W} \operatorname{tr}_{\operatorname{End}}\left(-i \rho_{t, \varepsilon}^{*} \wedge \rho_{t, \varepsilon} \wedge \omega_{t, \varepsilon}^{n-1}\right)=0 .
$$

As $-i \rho_{t, \varepsilon}^{*} \wedge \rho_{t, \varepsilon} \wedge \omega_{t, \varepsilon}^{n-1}$ and $-i \rho_{\widetilde{H}}^{*} \wedge \rho_{\widetilde{H}} \wedge \omega_{\widetilde{H}}^{n-1}$ are top forms with values in the bundle of Hermitian semipositive endomorphisms of $\widetilde{F}$, the Fatou lemma shows 
that $-i \rho_{\widetilde{H}}^{*} \wedge \rho_{\widetilde{H}} \wedge \omega_{\widetilde{H}}^{n-1}$ and hence the second fundamental form vanish identically,

$$
\rho_{\widetilde{H}}=0 \quad \text { on } \widetilde{X} \backslash(D \cup W) .
$$

This has two consequences. First, by [Dem12, IV. Prop. 14.9] on $\widetilde{X} \backslash(D \cup W)$ one has a holomorphic splitting $\widetilde{E}=\widetilde{F} \oplus \widetilde{F}^{\perp}$. One can push that splitting down to $X$ to obtain a holomorphic splitting $E=F \oplus F^{\perp}$ on the big open subset $X_{\text {reg }} \backslash \pi(W)$ of $X$. By reflexivity, this direct sum decomposition extends to $X$.

Second, as $\mathscr{F}$ is a direct summand of $\mathscr{E}, F$ is a subbundle of $E$, and hence $\pi(W) \cap$ $X_{\text {reg }}=\varnothing$. In fact, we even have $W=\varnothing$. Consequently, (9.17.1) implies that the second fundamental form of $F$ in $E$ vanishes, from which parallelism follows by definition, cf. [Dem12, V. Prop. 14.3].

Step 9. Proof of Item (8.1.2). Let $\mathscr{F}$ be a direct summand of $\mathscr{E}$. Necessarily, $\mu_{H}(\mathscr{F})=0$. By Claim 9.17, the bundle $F:=\left.\mathscr{F}\right|_{X_{\text {reg }}}$ is holomorphically complemented and parallel. Therefore, $F_{x} \subset E_{x}$ is a $G$-invariant complex subspace by the holonomy principle. We are left to prove that the parallel transport of a $G$-invariant subspace of $E_{x}$ induces a holomorphic subbundle of $E$ over $X_{\text {reg }}$ that extends to $X$ as a direct summand of $\mathscr{E}$. This follows from Proposition 4.9 coupled with the observation that $G$ is unitary, so that the orthogonal complement of a $G$-invariant complex subspace of $E_{x}$ is still $G$-invariant.

\section{PROOF OF THEOREM 8.2 ("BOCHNER PRINCIPLE FOR TENSORS")}

First, observe that parallel transport of a $G$-invariant $\mathbb{C}$-linear tensor $t \in E_{x}$ induces a parallel section $\tau$ of $E$. As the $(0,1)$-part of the connection coincides with the holomorphic structure $\bar{\partial}_{E}$ on $E$, we have $\bar{\partial}_{E}(\tau)=0$, so that $\tau$ is holomorphic, see Reminder 2.7. As $\mathscr{E}$ is reflexive, the corresponding coherent analytic sheaf $\mathscr{E}$ an over $X^{a n}$ is likewise reflexive, see for example [GKP14, Lem. 2.16]. It follows that $\tau$ yields an element of $H^{0}\left(X^{a n}, \mathscr{E} a n\right)$ and hence of $H^{0}(X, \mathscr{E})$ by GAGA. Therefore, every $G$-invariant element of $E_{x}$ produces a section of $\mathscr{E}$ over $X$.

For the converse, we need to show that the evaluation $\sigma_{x}$ of any section $\sigma \in$ $H^{0}(X, \mathscr{E})$ is a $G$-invariant element of $E_{x}$. The proof is carried out in two steps.

Step 1: Proof if $G$ is connected. Let $\sigma \in H^{0}(X, \mathscr{E}) \backslash\{0\}$ and let $\mathscr{F}$ be the saturation in $\mathscr{E}$ of the trivial subsheaf $\langle\sigma\rangle \subset \mathscr{E}$ generated by $\sigma$. We claim that codim $\operatorname{supp}(\mathscr{F} /\langle\sigma\rangle) \geq 2$. Indeed, otherwise one would have $\mu_{H}(\mathscr{F})>$ $\mu_{H}(\langle\sigma\rangle)=0$, which would contradict the semistability of $\mathscr{E}$ with respect to $H^{n-1}$ proved in Item (8.1.1) of Theorem 8.1. From this, we conclude that $\langle\sigma\rangle$ coincides with $\mathscr{F}$, as both sheaves are reflexive and agree on a big open subset of $X$. In other words, $\langle\sigma\rangle$ is saturated, and hence a direct summand of $\mathscr{E}$, cf. Claim 9.17 on the previous page for detailed arguments of this. It therefore follows from Item (8.1.2) of Theorem 8.1 that $\sigma_{x}$ generates a $G$-invariant complex line in $E_{x}$.

As $G$ is connected, it follows from Proposition 5.3 that $G$ is a product of SU's and $S p^{\prime}$ s. In particular, $G$ is semisimple and therefore equal to its own commutator subgroup, [Bum04, Thm. 23.2]. It follows that every homomorphism $\chi: G \rightarrow \mathbb{C}^{*}$ is trivial. As a result, every $G$-invariant line in $E_{x}$ has to be point-wise fixed, and hence $\sigma_{x} \in E_{x}$ is fixed by $G$, as claimed.

Step 2: Proof in general. Theorem 7.1 provides us with a holonomy cover, that is, a quasi-étale morphism $\gamma: Y \rightarrow X$ such that $\operatorname{Hol}\left(Y_{\text {reg }}, g_{H_{Y}}\right)$ is connected, for $H_{Y}:=\gamma^{*} H$. Set

$$
\mathscr{E}_{Y}:=\left(\mathscr{T}_{Y}^{\otimes p} \otimes\left(\mathscr{T}_{Y}^{*}\right)^{\otimes q}\right)^{* *}
$$


and take $\sigma \in H^{0}(X, \mathscr{E})$. Let $Y^{\circ}:=\gamma^{-1}\left(X_{\text {reg }}\right)$. Then, $\left.\gamma\right|_{\gamma^{\circ}}: Y^{\circ} \rightarrow X_{\text {reg }}$ is a locally biholomorphic map between complex manifolds, so there is a well-defined pull back tensor $\left(\left.\gamma\right|_{Y^{\circ}}\right)^{*}\left(\left.\sigma\right|_{X_{\text {reg }}}\right) \in H^{0}\left(Y^{\circ}, \mathscr{E}_{Y}\right)$ which extends to a section $\sigma_{Y}$ of $\mathscr{E}_{Y}$ on the whole of $Y$. By Step 1 and the holonomy principle, $\left.\widetilde{\sigma}\right|_{Y_{\text {reg }}}$ is parallel with respect to $g_{H_{\gamma}}$, see Reminder 2.7. It follows from the universal property of the EGZ construction, Proposition 3.5, that on $\gamma^{\circ}$ we have $\gamma^{*} \omega_{H}=\omega_{H_{Y}}$, which induces the analogous equality for the associated Riemannian metrics. The claim follows from the holonomy principle together with the observation that vanishing of covariant derivatives and hence parallelism is a local property. This concludes the proof of Theorem 8.2.

\section{AUgMENTED IRREGULARITY REVISITED}

Combining our findings on covering constructions and Bochner principles, we obtain two new characterisations of varieties with (non-)vanishing augmented regularity, which do not rely on the computation of invariants on quasi-étale covers, but only on invariants of the variety under investigation. Additionally, we use the Bochner principle to provide two criteria for detecting finite quotients of Abelian varieties.

Theorem 11.1 (Augmented regularity and symmetric differentials). In the Standard setting 4.1, the following are equivalent.

(11.1.1) The augmented irregularity does not vanish: $\widetilde{q}(X) \neq 0$.

(11.1.2) The restricted holonomy leaves a non-zero vector of $V$ invariant: $V_{0} \neq\{0\}$.

(11.1.3) There exists a non-trivial symmetric differential on $X$. In other words, there exists $m \in \mathbb{N}^{+}$such that $h^{0}\left(X, \operatorname{Sym}^{[m]} \Omega_{X}^{1}\right) \neq 0$.

Remark 11.2. In the smooth case, similar results were proven by Kobayashi in [Kob80, Thm. 6]. Following the argumentation of [Kob80, Thm. 6 and 7], our methods even give an upper bound for the number of symmetric differentials, $h^{0}\left(X, \operatorname{Sym}^{[m]} \Omega_{X}^{1}\right) \leq\left(\begin{array}{c}m+\widetilde{q}(X)-1 \\ m\end{array}\right)$ for all $m>0$.

Remark 11.3. We emphasise that the equivalence "(11.1.1) $\Leftrightarrow(11.1 .3)$ " gives a purely algebro-geometric characterisation of non-vanishing augmented regularity in terms of invariants of $X$ alone. This underlines yet again the importance of this concept in the structure theory of klt varieties with numerically trivial canonical divisor.

As a corollary we obtain the following vanishing theorem, which generalises [Tia96, Thm. 2.1(2)] to our setup.

Corollary 11.4 (Detecting finite quotients of Abelian varieties, II). Let X be a klt variety with numerically trivial canonical divisor. Assume that $\mathscr{T}_{X}$ is stable with respect to some ample $\mathbf{Q}$-divisor. Then,

$$
h^{0}\left(X, \operatorname{Sym}^{[m]} \Omega_{X}^{1}\right)=0 \quad \text { for all } m \in \mathbb{N}^{+},
$$

unless $X$ is of the form $A / G$ where $A$ is an Abelian variety and $G$ is a finite group whose action on $A$ is free in codimension one.

Proof. If $h^{0}\left(X, \operatorname{Sym}^{[m]} \Omega_{X}^{1}\right) \neq\{0\}$, it follows from Theorem 11.1 that $V_{0} \neq\{0\}$. As $\mathscr{T}_{X}$ is stable, using Corollary 6.8 we see that the representation of the full holonomy group $G$ on $V$ is irreducible. Looking at Observation 6.2 we conclude that $V_{0}=W_{0}=V$. Therefore, Theorem 7.1 implies that there exists a quasi-étale cover $\gamma^{\prime}: A^{\prime} \rightarrow X$, where $A^{\prime}$ is an Abelian variety. By taking Galois closure of $\gamma^{\prime}$, [GKP16c, App. B in the preprint version], and observing that finite étale covers of 
Abelian varieties are Abelian varieties themselves, we conclude that there exists an Abelian variety $A$ together with a quasi-étale Galois cover $\gamma: A \rightarrow X$.

Remark 11.5. Setup as in Corollary 11.4. If non-zero symmetric differentials exist on $X$, the cotangent sheaf is flat with finite monodromy and stable, but not strongly stable. The group $G$ is isomorphic to the holonomy group of a singular KählerEinstein metric on $X$, and the holonomy representation of $G$ on the fibre over some smooth point of $X$ is irreducible. Smooth examples exhibiting this behaviour can be found in [OS01].

11.1. Preparation for the proof of Theorem 11.1. We note the following two simple representation-theoretic lemmata.

Lemma 11.6. Let $n \geq 2$. Let $G=\operatorname{SU}(n)$ or let $n$ be even and $G=\operatorname{Sp}\left(\frac{n}{2}\right)$. Let $W$ be the complex standard representation of $G$. Then, $\left(\operatorname{Sym}^{m} W^{*}\right)^{G}=\{0\}$ for all $m \geq 0$.

Proof. We have $\left(\operatorname{Sym}^{m} W^{*}\right) \cong \mathbb{C}[W]_{m}$ as $G^{\mathbb{C}}$-representations. Moreover, $W$ does not admit any non-constant $G^{\mathbb{C}}$-invariant (homogeneous) polynomial, as in both cases $G^{\mathbb{C}}=\operatorname{SL}(n, \mathbb{C})$ or $\operatorname{Sp}\left(\mathbb{C}^{n}, \omega_{s t d}\right)$, respectively, has an open orbit in $W$. Consequently, $\left(\operatorname{Sym}^{m}\left(W^{*}\right)^{G} \cong \mathbb{C}[W]_{m}^{G}=\mathbb{C}[W]_{m}^{G^{C}}=\{0\}\right.$, as claimed.

Lemma 11.7. Let $\Gamma$ be a finite group of order $m:=\# \Gamma$ and let $V \neq\{0\}$ be a finitedimensional complex $\Gamma$-representation. Then, $\left(\operatorname{Sym}^{m} V\right)^{\Gamma} \neq\{0\}$.

Proof. Let $\left\{e=\gamma_{0}, \gamma_{1}, \ldots, \gamma_{m-1}\right\}$ be the elements of $\Gamma$ and let $\pi: V^{\otimes m} \rightarrow$ $\left(\right.$ Sym $\left.^{m} V\right)$ be the $\Gamma$-equivariant projection map, $v_{0} \otimes \cdots \otimes v_{m-1} \mapsto v_{0} \odot \cdots \odot v_{m-1}$. Pick any non-zero vector $v \in V$. Then, $0 \neq v \odot \gamma_{1}(v) \odot \cdots \odot \gamma_{m-1}(v) \in$ $\left(\operatorname{Sym}^{m} V\right)^{\Gamma}$.

\subsection{Proof of Theorem 11.1. -}

Equivalence (11.1.1) $\Leftrightarrow$ (11.1.2). This follows from Item (7.2.2) of Corollary 7.2.

Implication (11.1.2) $\Rightarrow$ (11.1.3). The action of $G$ on $V_{0}$ is given by the representation of a finite group $\Gamma$ on $V_{0}$, see Item (7.2.1) of Corollary 7.2. Set $m:=\# \Gamma$. Hence, by Lemma 11.7, there exists a G-invariant non-zero vector in $\left(\operatorname{Sym}^{m} V_{0}^{*}\right) \subset$ $\left(\operatorname{Sym}^{m} V^{*}\right)$. The Bochner principle for reflexive tensors, Theorem 8.2, then implies that $H^{0}\left(X, \operatorname{Sym}^{[m]} \Omega_{X}^{1}\right) \neq\{0\}$, as claimed.

Implication $\neg(11.1 .2) \Rightarrow \neg(11.1 .3)$. We suppose that $V_{0}=\{0\}$ and let $\sigma \in$ $H^{0}\left(X, \operatorname{Sym}^{[p]} \Omega_{X}^{1}\right)$ for some $p>0$. We aim to show that $\sigma$ vanishes identically. The Bochner principle for tensors, Theorem 8.2, implies that $\sigma_{x} \in\left(\operatorname{Sym}^{p} V^{*}\right)^{G^{\circ}}$. As the action of $G^{\circ}$ on $V^{*}$ is totally decomposed, cf. Construction 5.1, and as $V_{0}=\{0\}$, the standard decomposition of the symmetric product of a direct sum of representations, [BtD85, Chap. II, (3.1)], yields

$$
\left(\operatorname{Sym}^{p} V^{*}\right)^{G^{\circ}}=\bigoplus_{\substack{k_{1}, \ldots, k_{m} \in \mathbb{N} \\ \Sigma k_{j}=p}}\left(\operatorname{Sym}^{k_{1}} V_{1}^{*}\right)^{G_{1}^{\circ}} \otimes \cdots \otimes\left(\operatorname{Sym}^{k_{m}} V_{m}^{*}\right)^{G_{m}^{\circ}}
$$

Set $n_{i}:=\operatorname{dim} V_{i}$ and recall from Proposition 5.3 for each $i=1, \ldots, m$, either $G_{i}^{\circ} \cong$ $\mathrm{SU}\left(n_{i}\right)$, or $n_{i}$ is even and $G_{i}^{\circ} \cong \operatorname{Sp}\left(\frac{n_{i}}{2}\right)$. In either case, observe that the action $G_{i}^{\circ} \circlearrowleft V_{i}^{*}$ is isomorphic to the dual of the standard action of the respective group. Lemma 11.6 hence implies that $\sigma_{x} \in\left(\operatorname{Sym}^{p} V^{*}\right)^{G^{\circ}}=\{0\}$. We conclude that $\sigma=0$, as desired. 


\section{Part IV. Varieties with strongly stable tangent sheaf}

\section{THE BASIC DICHOTOMY: CY AND IHS}

If $X$ is a smooth, simply connected, irreducible compact Kähler manifold with trivial first Chern class, then $X$ is either an irreducible Calabi-Yau manifold or an irreducible holomorphic symplectic variety, where these two classes are distinguished by the algebra of holomorphic forms. The goal of this section is to show that after passing to a quasi-étale cover, any projective klt variety with numerically trivial canonical divisor and strongly stable tangent sheaf falls into one of the two classes introduced in Definition 1.3. We will also give the proof of Proposition F and relate our discussion to algebraic holonomy, a concept introduced by Balaji and Kollár in [BK08].

12.1. Differential forms on varieties with strongly stable tangent sheaf. From the irreducible case of the general results obtained in Part II, we obtain the following description of varieties with strongly stable tangent sheaf.

Theorem 12.1 (Holonomy dichotomy for strongly stable varieties). Assume the standard Setting 4.1. Then, the sheaf $\mathscr{T}_{X}$ is strongly stable if and only if the restricted holonomy group is one of the following two groups and the action of $G^{\circ}$ on $V$ is the standard action of the respective group.

(12.1.1) The group $G^{\circ}$ is isomorphic to $\mathrm{SU}(n)$.

(12.1.2) The dimension $n$ is even, and the group $G^{\circ}$ is isomorphic to $\operatorname{Sp}\left(\frac{n}{2}\right)$.

In either case, there exists a quasi-étale cover $\gamma: Y \rightarrow X$ such that restricted holonomy and holonomy agree on $Y$. More precisely, using Notation 4.4 we have $G^{\circ}=G_{Y}^{\circ}=G_{Y}$.

Proof. Recall from Corollary 7.4 that $\mathscr{T}_{X}$ is strongly stable if and only if the restricted holonomy representation $G^{\circ} \circlearrowleft V$ is irreducible. Proposition 5.3 ("Classification of restricted holonomy") then yields the claimed dichotomy. The last claim is just the existence of the holonomy cover, Theorem 7.1.

Theorem 12.2 (Reflexive differentials on strongly stable varieties). In the standard Setting 4.1, assume that the sheaf $\mathscr{T}_{X}$ is strongly stable. Then, the spaces of holomorphic $p$-forms can be controlled as follows.

(12.2.1) If $G^{\circ}$ is isomorphic to $\mathrm{SU}(n)$, then

$$
h^{0}\left(X, \Omega_{X}^{[p]}\right) \leq \begin{cases}1 & \text { if } p=0 \text { or } p=n \\ 0 & \text { otherwise. }\end{cases}
$$

(12.2.2) If the dimension $n$ is even and $G^{\circ}$ is isomorphic to $\operatorname{Sp}\left(\frac{n}{2}\right)$, then

$$
h^{0}\left(X, \Omega_{X}^{[p]}\right) \leq \begin{cases}1 & \text { if } 0 \leq p \leq n \text { and if } p \text { is even } \\ 0 & \text { otherwise. }\end{cases}
$$

If $G=G^{\circ}$, then the inequalities are in fact equalities.

Remark 12.3. If $G^{\circ}$ is isomorphic to $\mathrm{SU}(n)$ and if $K_{X}$ is linearly trivial, then we already have $G=G^{\circ}$. In fact, if $K_{X} \sim 0$, then there exists a non-zero holomorphic top-form on $X_{\text {reg, }}$, hence by the Bochner principle, Theorem 8.2, we have $G \subset$ $\operatorname{SU}(n)$. But then $G=G^{\circ}=\mathrm{SU}(n)$.

Proof of Theorem 12.2. We handle both cases simultaneously. Let $\gamma: Y \rightarrow X$ be a quasi-étale cover such that holonomy and restricted holonomy of $Y_{\text {reg agree, as re- }}$ called in Theorem 12.1. The tangent sheaf $\mathscr{T}_{Y}$ is then likewise strongly stable, and the restricted holonomies of $Y_{\text {reg }}$ and $X_{\text {reg }}$ agree. We also observe that the reflexive pullback morphisms $\gamma^{[*]}: H^{0}\left(X, \Omega_{X}^{[p]}\right) \rightarrow H^{0}\left(Y, \Omega_{Y}^{[p]}\right)$ are injective for all $p$. In 
order to establish all claims made, it therefore remains to show that equality holds in Inequalities (12.2.1), (12.2.2) for $h^{0}\left(Y, \Omega_{Y}^{[p]}\right)$. The Bochner principle for tensors, Theorem 8.2, applies to show that the natural evaluation map establishes a linear isomorphism

$$
H^{0}\left(Y, \Omega_{Y}^{[p]}\right) \stackrel{\cong}{\longrightarrow}\left(\bigwedge^{p} V^{*}\right)^{G_{Y}} .
$$

In addition, we have seen in Reminder 2.7 that $\left(\wedge^{p} V^{*}\right)^{G_{Y}} \cong \overline{\left(\wedge^{p} V\right)^{G_{Y}}}$. The desired equalities hence follow from classical invariant theory and representation theory for the groups $\mathrm{SL}_{\mathrm{C}}(V)=\mathrm{SU}(n)^{\mathrm{C}}$ and $\mathrm{Sp}_{\mathrm{C}}(V)=\mathrm{Sp}\left(\frac{n}{2}\right)^{\mathrm{C}}$ : by [GW09, Thm. 5.5.11] the non-trivial $\mathrm{SL}_{\mathbb{C}}(V)$-representations $\wedge^{p} V$ are all irreducible and therefore do not contain non-zero invariant vectors; the computation of $\operatorname{Sp}_{\mathbb{C}}(V)$-invariants in $\wedge^{p} V$ is given in [GW09, Thm. 5.3.3].

Definition 12.4 (Holomorphic symplectic form). Let X be a normal variety. A reflexive differential two-form $\sigma \in H^{0}\left(X, \Omega_{X}^{[2]}\right)$ on $X$ is called holomorphic symplectic if

(12.4.1) $\left.\sigma\right|_{X_{\mathrm{reg}}}$ is everywhere non-degenerate,

(12.4.2) $\left.\sigma\right|_{X_{\text {reg }}}$ is closed: $d\left(\left.\sigma\right|_{X_{\text {reg }}}\right)=0$, and

(12.4.3) $\left.\sigma\right|_{X_{\text {reg }}}$ extends regularly to any resolution of singularities of $X$.

Lemma 12.5 (Two-forms are holomorphic symplectic). In the standard Setting 4.1, assume that the sheaf $\mathscr{T}_{X}$ is strongly stable. If there exists a non-vanishing form $0 \neq \sigma \in$ $H^{0}\left(X, \Omega_{X}^{[2]}\right)$, then $\sigma$ is holomorphic symplectic, and any other reflexive differential form on $X$ is a constant multiple of the appropriate wedge power $\sigma \wedge \cdots \wedge \sigma$ of $\sigma$.

Proof. The existence of $0 \neq \sigma \in H^{0}\left(X, \Omega_{X}^{[2]}\right)$ implies that we are in case (12.1.2) of Theorem 12.1. As $X$ is assumed to be projective and klt, the restriction of $\sigma$ to $X_{\text {reg }}$ extends to any resolution of $X$ and is therefore automatically closed, see [GKP14, Prop. 1.4]. The assertion that $\left.\sigma\right|_{X_{\text {reg }}}$ is everywhere non-degenerate has been shown in [GKP16b, Cor. 8.10]. Together with Item (12.2.2) of Theorem 12.2 we conclude that every reflexive differential form on $X$ is a constant multiple of a wedge power of $\sigma$.

Combine Corollary 7.4, Theorem 12.2, and Lemma 12.5 to obtain the following.

Corollary 12.6. In the standard Setting 4.1, if $n$ is even and $G \cong \operatorname{Sp}\left(\frac{n}{2}\right)$, then $X$ carries a holomorphic symplectic form $\sigma$ with the following property: if $\gamma: Y \rightarrow X$ is any quasiétale cover, we have an isomorphism of algebras

$$
\bigoplus_{p=0}^{n} H^{0}\left(Y, \Omega_{Y}^{[p]}\right)=\mathbb{C}\left[\gamma^{[*]} \sigma\right] .
$$

12.2. Calabi-Yau and irreducible holomorphic symplectic varieties. One can reformulate the results obtained in the previous subsection using the terminology introduced in Definition 1.3 as follows.

Corollary 12.7 (Dichotomy for varieties with strongly stable tangent sheaf). In the standard Setting 4.1, assume that $\mathscr{T}_{X}$ is strongly stable. Then, one of the following cases occurs.

(12.7.1) The restricted holonomy group is equal to $\mathrm{SU}(n)$, and if $\gamma: Y \rightarrow X$ denotes a quasi-étale cover making $K_{Y} \sim 0$, then $Y$ is Calabi-Yau.

(12.7.2) The dimension of $X$ is even, the restricted holonomy group is equal to $\operatorname{Sp}\left(\frac{n}{2}\right)$, and if $\gamma: Y \rightarrow X$ denotes a quasi-étale cover making $G^{\circ}=G_{Y}^{\circ}=G_{Y}$, then $Y$ is irreducible holomorphic symplectic. 
Remark 12.8. If $n=2$, the definition of $\mathrm{CY}$ and IHS varieties coincide. However, if $n \geq 3$, then (12.7.1) and (12.7.2) are mutually exclusive. The tangent sheaf of a CY or IHS variety is strongly stable by [GKP16b, Prop. 8.20].

Remark 12.9 (Varieties with linearly trivial canonical divisor and strongly stable tangent sheaf). Corollary 12.7 implies that a normal projective variety with at worst canonical singularities, linearly trivial canonical divisor, and strongly stable tangent sheaf is either Calabi-Yau, or admits a finite, quasi-étale cover that is an irreducible holomorphic symplectic variety; Example 14.9 describes a variety with linearly trivial canonical divisor and no two-form that admits a quasi-étale cover that is IHS. This shows that in Item (12.7.2) above taking a quasi-étale cover in general cannot be avoided. The reader is encouraged to compare this observation with the smooth situation, see [GKP16b, Rem. 8.22].

12.3. Characterisation of IHS and CY varieties in terms of holonomy. We recall that the definition of CY and IHS varieties, Definition 1.3, is formulated in purely algebro-geometric terms. We are now in a position to give a complementary characterisation of these two types of varieties purely in terms of differential-geometric holonomy, as formulated in Proposition F and in complete accordance with the smooth theory.

Proposition 12.10 (Characterisation of $\mathrm{CY}$ and IHS varieties in terms of holonomy). In the standard Setting 4.1, the following conditions are equivalent.

(12.10.1) $X$ is a Calabi-Yau variety.

(12.10.2) $\mathrm{Hol}\left(X_{\mathrm{reg}}, g_{H}\right)$ is connected and $H^{0}\left(X, \Omega_{X}^{[p]}\right)=\{0\}$ for all $0<p<n$.

(12.10.3) $\mathrm{Hol}\left(X_{\mathrm{reg}}, g_{H}\right)$ is isomorphic to $\mathrm{SU}(n)$.

Analogously, the following conditions are equivalent.

(12.10.4) $X$ is an irreducible holomorphic symplectic variety.

(12.10.5) $\mathrm{Hol}\left(X_{\mathrm{reg}}, g_{H}\right)$ is connected, and there exists a holomorphic symplectic two-form $\sigma \in H^{0}\left(X, \Omega_{X}^{[2]}\right)$ such that $\oplus_{p=0}^{n} H^{0}\left(X, \Omega_{X}^{[p]}\right)=\mathbb{C}[\sigma]$.

(12.10.6) $\mathrm{Hol}\left(X_{\mathrm{reg}}, g_{H}\right)$ is isomorphic to $\operatorname{Sp}\left(\frac{n}{2}\right)$.

Proof. Notice first that any of the conditions (12.10.1)-(12.10.6) implies that $K_{X}$ is linearly equivalent to zero, and in particular that $X$ has canonical singularities. This is clear for (12.10.1), (12.10.4) and (12.10.5). For (12.10.3) and (12.10.6), this is a consequence of the Bochner principle for reflexive forms, Theorem 8.2. Finally, for (12.10.2) this follows from Proposition 5.3 and the Bochner principle for reflexive forms.

So in any case, there exists a nowhere vanishing $n$-form on $X$. The Bochner principle hence implies that $G \subseteq \mathrm{SU}(n)$. Using the notation of Proposition 5.3 ("Classification of restricted holonomy"), we obtain that $G^{\circ}=\times_{i=1}^{m} G_{i}^{\circ}$ acts as a product, and if $n_{i}=\operatorname{dim} V_{i}$ then either $G_{i}^{\circ}=\operatorname{SU}\left(n_{i}\right)$ or $n_{i}$ is even and $G_{i}^{\circ}=\operatorname{Sp}\left(\frac{n_{i}}{2}\right)$.

Implication (12.10.1) $\Rightarrow$ (12.10.3). We know that $G^{\circ} \subseteq G \subseteq \mathrm{SU}(n)$ and we need to prove that equality holds in both steps. Using Theorem 7.1, one can find a quasi-étale holonomy cover $\gamma: Y \rightarrow X$ such that $G_{Y}=\times_{i=1}^{m} G_{i}^{\circ}$. By the Bochner principle for reflexive forms, this yields $m$ independent reflexive holomorphic forms of positive degree on $Y$. Given the restrictions on the algebra of reflexive differential forms on $Y$ dictated by the $\mathrm{CY}$ condition, one gets successively that $m=1$ and $G^{\circ}=\mathrm{SU}(n)$. The conclusion follows.

Implication (12.10.3) $\Rightarrow$ (12.10.2). This is a direct application of the Bochner principle for reflexive forms. 
Implication (12.10.2) $\Rightarrow$ (12.10.1). We have $G^{\circ}=G \subseteq \mathrm{SU}(n)$. Using Proposition 5.3, one gets that for every $1 \leq i \leq m, G_{i}=G_{i}^{\circ}$ is either $\operatorname{SU}\left(n_{i}\right)$ or $\operatorname{Sp}\left(\frac{n_{i}}{2}\right)$, where $n_{i}=\operatorname{dim} V_{i}$. By the Bochner principle for reflexive forms, we have that $h^{0}\left(X, \Omega_{X}^{\left[n_{i}\right]}\right) \geq 1$, which implies that for all $1 \leq i \leq m$, one either has $n_{i}=0$ or $n_{i}=n$. In particular, $m=1$. Moreover, given the restrictions on the algebra of reflexive differential forms on $X$ dictated by the $C Y$ condition, $G$ cannot be the unitary symplectic group; it follows that $G=\mathrm{SU}(n)$. Now, if $\gamma: Y \rightarrow X$ is any quasi-étale cover, then $G_{Y}^{\circ}=G^{\circ}=\operatorname{SU}(n)$, and hence the Bochner principle implies that $X$ is $C Y$.

Implication (12.10.4) $\Rightarrow$ (12.10.6). By the Bochner principle, $G^{\circ} \subseteq G \subseteq \operatorname{Sp}\left(\frac{n}{2}\right)$ and we need to prove the previous inclusions are equalities. A short computation shows that all $n_{i}$ 's are even and $G_{i}^{\circ} \subseteq \operatorname{Sp}\left(n_{i} / 2\right)$. Using Theorem 7.1, one can then find a quasi-étale holonomy cover $\gamma: Y \rightarrow X$ such that $G_{Y}=X_{i=1}^{m} \operatorname{Sp}\left(\frac{n_{i}}{2}\right)$. By the Bochner principle, this yields $m$ independent reflexive holomorphic two-forms on $Y$. As $X$ is IHS, this implies that $m=1$ and $G^{\circ}=\operatorname{Sp}\left(\frac{n}{2}\right)$.

Implication (12.10.6) $\Rightarrow(12.10 .5)$. Again, this is a direct application of the Bochner principle for reflexive forms.

Implication $(12.10 .5) \Rightarrow(12.10 .4)$. First, the Bochner principle shows that one has $G^{\circ}=G \subset \operatorname{Sp}\left(\frac{n}{2}\right)$. As in the Implication "(12.10.2) $\Rightarrow(12.10 .1)^{\prime \prime}$ above, one finds that the $n_{i}$ 's are even and that $G_{i}=G_{i}^{\circ}=\operatorname{Sp}\left(\frac{n_{i}}{2}\right)$. By the Bochner principle again, $h^{0}\left(X, \Omega_{X}^{[2]}\right)=m$. From this it follows that $m=1$, that is, $G=\operatorname{Sp}\left(\frac{n}{2}\right)$. Now, if $\gamma: Y \rightarrow X$ is any quasi-étale cover, then $G_{Y}^{\circ}=G^{\circ}=\operatorname{Sp}\left(\frac{n}{2}\right)$ and the Bochner principle shows that $X$ is IHS.

This concludes the proof of Proposition F.

12.4. Characterisation in terms of algebraic holonomy groups. In this section, we characterise the two cases of the dichotomy in terms of stability properties of powers of the (co)tangent sheaf.

12.4.1. Holonomy groups of stable bundles. We start by giving a quick introduction to the theory of algebraic holonomy groups of stable reflexive sheaves, as developed in [BK08]. We follow [Dru18, Sect. 6.19] and refer the reader to these two sources for references of the classical results mentioned below.

Theorem and Definition 12.11 (Algebraic holonomy). Let $X$ be a normal projective variety, and let $\mathscr{E}$ be a reflexive sheaf on $X$, locally free away from a small subset $B \subset X$. Suppose that $\mathscr{E}$ is stable and of slope $\mu_{H}(\mathscr{E})=0$ with respect to an ample Cartier divisor $H$ on $X$. Let $x \in X_{\text {reg }} \backslash B$ and let $\mathscr{E}_{x}$ be the fibre of $\mathscr{E}$ over $x$.

Then, there exists a unique smallest subgroup $H_{x}(\mathscr{E}) \subset \mathrm{GL}_{\mathbb{C}}\left(\mathscr{E}_{x}\right)$, called algebraic holonomy group of $\mathscr{E}$ at $x$, such that the following holds. For every smooth, pointed, projective curve $(D, y)$ and every pointed morphism $g:(D, y) \rightarrow(X, x)$ where $\mathscr{E}$ is locally free along $g(D)$ and where $g^{*}(\mathscr{E})$ is polystable (and hence unitary flat by a theorem of Narasimhan and Seshadri), the image of the resulting representation of $\pi_{1}(D, y) \rightarrow$ $\mathrm{GL}_{\mathbb{C}}\left(\left(g^{*} \mathscr{E}\right)_{y}\right)=\mathrm{GL}_{\mathbb{C}}\left(\mathscr{E}_{x}\right)$ is contained in $H_{x}(\mathscr{E})$.

We emphasise that in contrast to the discussion of differential-geometric holonomy groups in previous parts of the paper, the above construction is algebraic.

Remark 12.12. Refining the restriction theorem of Mehta and Ramanathan, Graf has shown the following in [Gra16, Thm. 1.1]. If $m \gg 0$ is sufficiently divisible and $C \subset X_{\text {reg }} \backslash B$ is a curve containing $x$ that is obtained as a complete intersection of sufficiently general elements of $|m H|$ passing through $x$, the restriction $\left.\mathscr{E}\right|_{C}$ 
is a stable vector bundle on $C$. Again by the theorem of Narasimhan-Seshadri, $\left.\mathscr{E}\right|_{C}$ corresponds to a unique unitary representation $\rho: \pi_{1}(C, x) \rightarrow U\left(\mathscr{E}_{x}\right)$ with respect to some Hermitian form on $\mathscr{E}_{x}$. Balaji and Kollár prove in [BK08, Thm. 20] that if $m \gg 0$ is sufficiently large and if $C$ is a sufficiently general complete intersection curve through $x \in X_{\text {reg }} \backslash B$, then the algebraic holonomy $H_{x}(\mathscr{E})$ equals the Zariski-closure of $\rho\left(\pi_{1}(C, x)\right)$ in $\mathrm{GL}_{C}\left(\mathscr{E}_{x}\right)$. Moreover, $H_{x}(\mathscr{E})$ is the smallest algebraic subgroup such that for every curve $x \in C \subset X$ such that $\left.\mathscr{E}\right|_{C}$ is stable, the image of the Narasimhan-Seshadri representation is contained in $H_{x}(\mathscr{E})$. In particular, $H_{x}(\mathscr{E})$ is independent of the ample divisor $H$.

Remark 12.13. Connectivity properties of the algebraic holonomy groups are closely connected to the question whether a given stable sheaf of degree zero is actually strongly stable, see [Dru18, Lem. 6.22].

The following Bochner principle for the algebraic holonomy group provides a link to differential-geometric holonomy groups.

Proposition 12.14 (Bochner principle for algebraic holonomy, [BK08, Thm. 20(3)]). Setup as in Definition 12.11. Then, for every $m, n \in \mathbb{N}$, the evaluation map gives a oneto-one correspondence between direct summands of the reflexive tensor product $(\mathscr{E} \otimes m \otimes$ $\left.\left(\mathscr{E}^{*}\right)^{\otimes n}\right)^{* *}$ and $H_{x}(\mathscr{E})$-invariant subspaces of $\mathscr{E}_{x}^{\otimes m} \otimes\left(\mathscr{E}_{x}^{*}\right)^{\otimes n}$.

Observing that Tannakian duality and the knowledge of a small number of representations determines a reductive group completely, [BK08, Sect. 4], one obtains the following result.

Proposition 12.15. Setup as in Definition 12.11. Assume additionally that $\operatorname{det} \mathscr{E}_{X} \cong \mathscr{O}_{X}$ and that $H_{x}(\mathscr{E})$ is connected. Then, the following are equivalent.

(12.15.1) For some (and a posteriori all) $m \geq 2$, the $m$-th reflexive symmetric power $\operatorname{Sym}^{[m]}(\mathscr{E}):=\operatorname{Sym}^{m}(\mathscr{E})^{* *}$ is indecomposable.

(12.15.2) The algebraic holonomy $H_{x}(\mathscr{E})$ is either $H_{x}(\mathscr{E}) \cong \operatorname{SL}_{\mathbb{C}}\left(\mathscr{E}_{x}\right)$, or $H_{x}(\mathscr{E}) \cong$ $\mathrm{Sp}_{\mathrm{C}}\left(\mathscr{E}_{x}\right)$ for a suitable complex-symplectic form on $\mathscr{E}_{x}$. In the second case, rank $\mathscr{E}$ is even.

Proof. From the proof of [BK08, Prop. 41], it follows that $H_{x}(\mathscr{E})$ is one of the following: $\mathrm{SL}_{\mathbb{C}}\left(\mathscr{E}_{x}\right), \mathrm{GL}_{\mathbb{C}}\left(\mathscr{E}_{x}\right), \operatorname{Sp}_{\mathbb{C}}\left(\mathscr{E}_{x}\right)$, or $\mathrm{GSp}_{\mathbb{C}}\left(\mathscr{E}_{x}\right)$. The two groups not appearing in our list are excluded by the Bochner principle, Proposition 12.14, and the assumption on the determinant of $\mathscr{E}$.

12.4.2. The basic dichotomy in terms of algebraic holonomy. Using the theory of algebraic holonomy groups summarised in the previous section, we may now give another characterisation of the two cases in the basic dichotomy.

Theorem 12.16. In the Standard setting 4.1, additionally assume that $\omega_{X} \cong \mathscr{O}_{X}$, that $\mathscr{T}_{X}$ is strongly stable, and that $G=G^{\circ}$. Then, the following holds.

(12.16.1) The variety $X$ is $C Y$ if and only if the connected component of $H_{x}\left(\mathscr{T}_{X}\right)$ is equal to $\mathrm{SL}_{\mathrm{C}}\left(T_{x} X\right)$.

(12.16.2) The variety $X$ is IHS if and only if the connected component of $H_{x}\left(\mathscr{T}_{X}\right)$ is equal to $\operatorname{Sp}_{\mathrm{C}}\left(T_{x} X\right)$, where the linear complex-symplectic form on $T_{x} X$ is the evaluation of the holomorphic symplectic two-form.

Remark 12.17. We thank Stéphane Druel for explaining the following to us: in the setup of Theorem 12.16, it can be shown by a more detailed differential-geometric analysis that the structure group of $\mathscr{T}_{\mathrm{X} \text { reg }}$ can be reduced to the complexification $G^{\mathrm{C}}$ of $G$, which is reductive as $G$ is compact. Therefore, by [BK08, Thm. 1(4)] the subgroup $H_{x}\left(\mathscr{T}_{X}\right) \subset G^{\mathbb{C}}$ is in fact already connected. 
Proof of Theorem 12.16. From [Dru18, Lem. 6.20] and the proof of [BK08, Lem. 40] it follows that there exists a quasi-étale cover $\gamma: Y \rightarrow X$ and a point $y \in Y$ mapping to $x$ such that $H_{y}\left(\gamma^{[*]} \mathscr{T}_{X}\right)=H_{y}\left(\mathscr{T}_{Y}\right)=H_{x}\left(\mathscr{T}_{X}\right)^{\circ}$. Note that $X$ is CY (resp. IHS) if and only if $Y$ is. Therefore one can assume without loss of generality that $H_{x}\left(\mathscr{T}_{X}\right)$ is connected.

We claim that Sym ${ }^{[2]} \mathscr{T}_{X}$ is indecomposable. Indeed, by the Bochner principle for bundles, Theorem 8.1, a direct summand would give rise to a $G$-stable subspace of the G-representation $\operatorname{Sym}^{2}\left(T_{x} X\right)$, which is irreducible by [FH91, Sect. 24.1 and 24.2], a contradiction.

We may hence apply Proposition 12.15 to conclude that $H_{x}\left(\mathscr{T}_{X}\right)$ is either $\mathrm{SL}_{\mathbb{C}}\left(T_{x} X\right)$ or $\mathrm{Sp}_{\mathbb{C}}\left(T_{x} X\right)$. We claim that the latter case occurs if and only if $X$ carries a holomorphic symplectic form. Indeed, if $H_{x}\left(\mathscr{T}_{X}\right)=\operatorname{Sp}_{\mathbb{C}}\left(T_{x} X\right)$, then the Bochner principle for algebraic holonomy, Proposition 12.14, implies that $\Omega_{X}^{[2]}$ has a onedimensional trivial direct summand; the corresponding two-form is holomorphic symplectic owing to Lemma 12.5. Conversely, if there exists a non-vanishing twoform on $X$, then the direct summand $\Omega_{X}^{[2]} \subset\left(\mathscr{T}_{X} \otimes \mathscr{T}_{X}\right)^{* *}$, which is polystable by Theorem 8.1, is decomposable. As a consequence, $\Lambda^{2}\left(T_{x}^{*} X\right)$ is decomposable as $H_{x}\left(\mathscr{T}_{X}\right)$-representation, which in turn excludes the algebraic holonomy from being equal to $\mathrm{SL}_{\mathrm{C}}\left(T_{x} X\right)$ by [GW09, Thm. 5.5.11].

\section{FUnDAMENTAL GROUPS}

This section is devoted to studying the fundamental group of Calabi-Yau and irreducible holomorphic symplectic varieties. In the smooth case, these varieties are by definition simply connected but in our singular setup, this might not be the case anymore. Actually, there are two relevant fundamental groups to look at, $\pi_{1}(X)$ and $\pi_{1}\left(X_{\text {reg }}\right)$, and we will obtain finiteness result concerning both of them.

13.1. Fundamental groups of even-dimensional $\mathrm{CY}$ and IHS varieties. The following theorem summarises our results for varieties of even dimension. Theorem 13.1 is expected to hold also in odd dimensions, and even more generally for varieties with vanishing augmented regularity, see [Kol95, Conj. 4.16] and [Cam95, Qu. 5.12]. Section 13.2 on the facing page contains partial results in this direction.

Theorem 13.1 (Fundamental groups of even-dim. strongly stable varieties). In the standard Setting 4.1, if $\mathscr{T}_{X}$ is strongly stable and if $\operatorname{dim} X$ is even, then $\pi_{1}(X)$ is finite. In particular, the topological fundamental group of an IHS variety is finite.

Proof. Let $\gamma: Y \rightarrow X$ be a global index-one cover, as given by Proposition 2.18. The image of $\pi_{1}(Y)$ in $\pi_{1}(X)$ has finite index by [Cam91, Prop. 1.3]. To prove finiteness of $\pi_{1}(X)$, it therefore suffices to show that $\pi_{1}(Y)$ is finite. As $Y$ has canonical singularities, this will follow from [GKP16b, Prop. 8.23] once we show that $\chi\left(Y, \mathscr{O}_{Y}\right) \neq 0$. First, we note that $\operatorname{dim} Y$ is even and that the tangent sheaf $\mathscr{T}_{Y}$ is strongly stable. In addition, [GKP16b, Prop. 6.9] shows that

$$
h^{q}\left(Y, \mathscr{O}_{Y}\right)=h^{0}\left(Y, \Omega_{Y}^{[q]}\right) \quad \text { for all } 0 \leq q \leq n,
$$

and so Theorem 12.2 yields that

$$
\chi\left(Y, \mathscr{O}_{Y}\right)=h^{0}\left(Y, \mathscr{O}_{Y}\right)+h^{0}\left(Y, \Omega_{Y}^{[2]}\right)+\cdots+(-1)^{n} \cdot h^{0}\left(Y, \Omega_{Y}^{[n]}\right) \geq 2 .
$$

This concludes the proof of Theorem 13.1.

Combining Theorem 13.1 and [GKP16b, Prop. 7.3] we obtain the following result. 
Corollary 13.2 (Étale fundamental groups of smooth locus). In the setting of Theorem 13.1, the étale fundamental group $\widehat{\pi}_{1}\left(X_{\mathrm{reg}}\right)$ is finite. In particular, the étale fundamental group of the smooth locus of any IHS variety is finite.

Corollary 13.3. Let $X$ be a $C Y$ variety of even dimension or an IHS variety. Then, $X$ is simply connected.

Proof. The argument follows [Bea83b, proof of Prop. 4(2)]. Let $\gamma: Y \rightarrow X$ be the universal cover of $X$ and let $d \in \mathbb{N}$ be its degree, which is finite by Theorem 13.1. Since klt spaces have rational singularities, the holomorphic Euler characteristics of $\mathscr{O}_{X}$ and $\mathscr{O}_{Y}$ can be computed by passing to a resolution of singularities and differ by a factor of $d$, that is, $\chi\left(Y, \mathscr{O}_{Y}\right)=d \cdot \chi\left(X, \mathscr{O}_{X}\right)$. On the other hand, as $Y$ is projective and $\gamma$ is étale, $Y$ is likewise $C Y$ or IHS, depending on the type of $X$. It follows from [GKP16b, Prop. 6.9] that

$$
\chi\left(Y, \mathscr{O}_{Y}\right)=\chi\left(X, \mathscr{O}_{X}\right)= \begin{cases}2 & \text { if } X \text { and } Y \text { are CY } \\ \frac{1}{2} \cdot \operatorname{dim} X+1 & \text { if } X \text { and } Y \text { are IHS }\end{cases}
$$

Either way, it follows that $d=1$. This concludes the proof.

Remark 13.4 (Corollary 13.3 is optimal). There are examples of smooth odddimensional CY-manifolds with non-trivial, finite fundamental group. For instance, there exists a fixed-point free action of $\mathbb{Z}_{5}$ on the diagonal quintic threefold in $\mathbb{P}^{4}$ such that the quotient has trivial canonical divisor.

Remark 13.5. The arguments and techniques of [OS11, Sect. 2] can be easily adapted to study klt varieties with numerically trivial canonical class that admit a quasi-étale cover by an IHS variety, or, in other words, to varieties whose restricted holonomy equals $\mathrm{Sp}\left(\frac{n}{2}\right)$. In analogy with the smooth case, one might call these Enriques varieties. An analogue of [GKP16b, Lem. 8.14] holds in this singular setup.

13.2. Fundamental groups of odd-dimensional $\mathrm{CY}$ varieties. After the discussion in the previous subsection, it remains to consider the fundamental group of odd-dimensional CY varieties. In the smooth case, it follows from the Theorem of Cheeger-Gromoll that such varieties have finite fundamental group, see [Bea83b, proof of Thm. 1]. Here, we gather some partial information for the singular case. All of these go back to Hodge-theoretical arguments and use the non-existence of reflexive symmetric differentials. In fact, they hold for arbitrary varieties with vanishing augmented irregularity.

Theorem 13.6 (Fundamental group of varieties with $\widetilde{q}=0$, I). Let $X$ be a projective klt variety with numerically trivial canonical divisor and vanishing augmented irregularity, $\widetilde{q}(X)=0$. Then, the following hold.

(13.6.1) The fundamental group $\pi_{1}(X)$ does not have any finite-dimensional representation with infinite image (over any field).

(13.6.2) For each $n \in \mathbb{N}$, the fundamental group $\pi_{1}(X)$ has only finitely many $n$ dimensional complex representations up to conjugation.

(13.6.3) If infinite, the group $\pi_{1}(X)$ cannot have weakly polynomial growth in the sense of [Kle10, Def. 1.1]

Remark 13.7. There exist finitely generated, infinite groups that do not admit finitedimensional representations. One example is Higman's group, [Ber94, Ex. 1.1].

Remark 13.8. Concerning Item (13.6.3), cf. also the discussion in [Cam95, p. 500].

Proof of Theorem 13.6. Let $\pi: \widetilde{X} \rightarrow X$ be a resolution of $X$ and recall from [Tak03, Thm. 1.1] that $\pi_{1}(\tilde{X})=\pi_{1}(X)$. 
Proof of (13.6.1). Argue by contradiction and suppose that there exists a representation $\pi_{1}(\tilde{X}) \rightarrow \mathrm{GL}_{r}(\mathbb{K})$ with infinite image, for some positive integer $r$ and some field $\mathbb{K}$. It then follows from a recent result of Brunebarbe-Klingler-Totaro, [BKT13, Thm. 0.1], that there exists a number $m>0$ and a non-zero element

$$
0 \neq \widetilde{\sigma} \in H^{0}\left(\widetilde{X}, \operatorname{Sym}^{m} \Omega_{\widetilde{X}}^{1}\right) .
$$

Restricting $\widetilde{\sigma}$ to the complement of the exceptional divisor of $\pi$ we obtain a nontrivial element $\sigma \in H^{0}\left(X, \operatorname{Sym}^{[m]} \Omega_{X}^{1}\right)$, which by Theorem 11.1 yields $\widetilde{q}(X) \neq 0$, contradiction.

Proof of (13.6.3). Item (13.6.3) follows from (13.6.1) and from the (deep) fact that an infinite, finitely generated group with weakly polynomial growth admits a finitedimensional (real) representation with infinite image, cf. [Kle10, Sect. 4].

Proof of (13.6.2). We saw in the proof of (13.6.1) that $H^{0}\left(\widetilde{X}, \operatorname{Sym}^{m} \Omega_{\widetilde{X}}^{1}\right)=\{0\}$ for all $m>0$. It hence follows from [Ara02, Prop. 2.4] or [Kli13, Thm. 1.6(i)] that the variety $\operatorname{Hom}\left(\pi_{1}(X), \mathrm{GL}_{r}(\mathbb{C})\right) / / \mathrm{GL}_{r}(\mathbb{C})$, which parametrises representations up to Jordan-Hölder equivalence, consists of finitely many points. By Item (13.6.1) and Maschke's Theorem every complex representation of $\pi_{1}(X)$ is in fact semisimple, in which case Jordan-Hölder equivalence reduces to equivalence up to conjugation. This establishes the claim made in item (13.6.2).

Remark 13.9. The converse of Item (13.6.1) is however false. The singular Kummer surface $X=A /\langle \pm 1\rangle$ that will be discussed in Example 14.1 satisfies $\widetilde{q}(X)=2$ but $\pi_{1}(X)=\{1\}$. Indeed, the minimal resolution of $X$ is a $K 3$ surface, hence it is simply connected and therefore so is $X$ by Takayama's result [Tak03, Thm. 1.1].

Corollary 13.10 (Fundamental group of varieties with $\widetilde{q}=0$, II). Let $X$ be a projective klt variety with numerically trivial canonical divisor and vanishing augmented irregularity. Then, the following hold.

(13.10.1) The fundamental group $\pi_{1}\left(X_{\mathrm{reg}}\right)$ does not have any finite-dimensional representation with infinite image (over any field).

(13.10.2) For each $n \in \mathbb{N}$, the fundamental group $\pi_{1}\left(X_{\text {reg }}\right)$ has only finitely many $n$ dimensional complex representations up to conjugation.

(13.10.3) If infinite, the group $\pi_{1}\left(X_{\mathrm{reg}}\right)$ cannot have weakly polynomial growth in the sense of [Kle10, Def. 1.1].

Proof. -

Proof of (13.10.1). Let $\gamma: Y \rightarrow X$ be a Galois, maximally quasi-étale cover, as constructed in [GKP16c, Thm. 1.5]. As $\gamma$ is quasi-étale, the Galois group G of $\gamma$ fits into an exact sequence as follows

$$
1 \rightarrow \pi_{1}\left(Y_{\text {reg }}\right) \stackrel{\gamma_{*}}{\longrightarrow} \pi_{1}\left(X_{\text {reg }}\right) \rightarrow G \rightarrow 1 .
$$

We let $\iota: Y_{\text {reg }} \hookrightarrow Y$ denote the inclusion map. Let $\rho: \pi_{1}\left(X_{\text {reg }}\right) \rightarrow \mathrm{GL}_{r}(\mathbb{K})$ be any representation of $\pi_{1}\left(X_{\text {reg }}\right)$ over any field $\mathbb{K}$. As $Y$ is maximally quasi-étale, it follows from [GKP16c, Sect. 8.1] or [Gro70, Thm. 1.2] there exists a representation $\rho_{Y}: \pi_{1}(Y) \rightarrow \mathrm{GL}_{r}(\mathbb{K})$ making the following diagram commutative

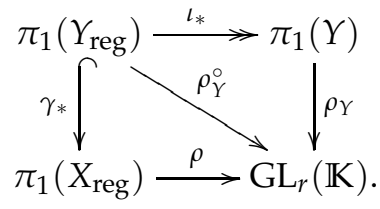


Let $\Gamma:=\operatorname{img}(\rho)$. Furthermore, as $\widetilde{q}(Y)=0$, we may apply part (13.6.1) of Theorem 13.6 to conclude that $\Gamma_{Y}:=\operatorname{img}\left(\rho_{Y}^{\circ}\right)=\operatorname{img}\left(\rho_{Y}\right)$ is a finite, normal subgroup of $\Gamma$. Using the exact sequence (13.10.4), from $\rho$ we obtain a surjective group homomorphism $\bar{\rho}: G \rightarrow \Gamma / \Gamma_{Y}$. As $G$ is finite by definition, $\Gamma / \Gamma_{Y}$ is hence finite. Together with the finiteness of $\Gamma_{Y}$ observed above, Item (13.10.1) follows.

Proof of (13.10.3). The claim follows as in the proof of Item (13.6.3) above.

Proof of (13.10.2). Fix $n \in \mathbb{N}$ and set $\Sigma:=\pi_{1}\left(X_{\text {reg }}\right)$ and $\Sigma_{Y}:=\pi_{1}\left(Y_{\text {reg }}\right)$. As every finite-dimensional complex representation of $\Sigma$ is semisimple by (13.10.1) and Maschke's Theorem, it suffices to show the claim for simple $\Sigma$-representations. Because each $\Gamma_{Y}$-representation factors over $\pi_{1}(Y)$, Theorem 13.6 implies that the group $\Sigma_{Y}$ has only finitely many $n$-dimensional complex representations up to conjugation. Therefore, if $\rho: \Sigma \rightarrow \mathrm{GL}_{\mathbb{C}}(V)$ is a simple $n$-dimensional representation, there are only finitely many possibilities for $\left.\rho\right|_{\Sigma_{\gamma}}$ up to conjugation. Consequently, again up to conjugation, there are only finitely many possibilities for $\operatorname{ind}_{\Sigma_{Y}} \sum_{Y}\left(\left.\rho\right|_{\Sigma_{Y}}\right)$, the $\Sigma$-module obtained via induction ${ }^{5}$ from $\Sigma_{Y}$ to $\Sigma$. There exists a natural $\Sigma$-module isomorphism

$$
\eta: \mathbb{C}\left[\Sigma / \Sigma_{Y}\right] \otimes_{\mathbb{C}} V \stackrel{\cong}{\longrightarrow} \operatorname{ind}_{\Sigma_{Y}}\left(\left.\rho\right|_{\Sigma_{Y}}\right),
$$

where $\mathbb{C}\left[\Sigma / \Sigma_{Y}\right]$ is the $\Sigma$-module of $\mathbb{C}$-valued functions on the homogeneous $\Sigma$ space $\Sigma / \Sigma_{Y}$, see [FH91, Ex. 3.6]. As the $\Sigma$-equivariant map $v \mapsto \eta(\underline{1} \otimes v)$ realises the simple representation $V$ as a direct summand of the semisimple representation $\operatorname{ind} \sum_{\Sigma_{\gamma}}\left(\left.\rho\right|_{\Sigma_{\gamma}}\right)$, there are only finitely many possibilities for $\rho$ up to isomorphism, as claimed in (13.10.2).

\section{EXAMPLES}

The present section gathers examples that illustrate the main results of this paper. As announced in the introduction, we begin in Section 14.1 with two examples that show how the holonomy changes under birational transformation. Perhaps more importantly, Section 14.2 illustrates the classification scheme established in the previous sections, underlines the necessity of using quasi-étale covers, and points out the differences to other suggestions for a definition of "irreducible holomorphic symplectic variety" that are found in the literature. Finally, Section 14.3 discusses moduli of sheaves on K3 surfaces.

14.1. Change of holonomy under crepant resolutions. The singular KählerEinstein metric $\omega_{H}$ discussed in the standard Setting 4.1 does depend on the choice of the ample divisor $H$. However, we have seen in Proposition $C$ that the isomorphism class of the restricted holonomy group $G^{\circ}$ is in fact independent of $H$. We can therefore speak of the restricted holonomy, and ask how it changes under birational transformation. The following two examples show that holonomy does in fact change, even for crepant resolutions of singularities.

Example 14.1 (Singular Kummer surface). Let $X:=A /\langle \pm 1\rangle$ where $A$ is an Abelian surface, and let $\pi: \widetilde{X} \rightarrow X$ be the (crepant) minimal resolution of $X$, which is a $K 3$ surface. We analyse the relevant (singular) Kähler-Einstein metrics.

On the crepant resolution: If $\omega_{\widetilde{X}}$ is any Ricci-flat Kähler metric on $\widetilde{X}$ with associated Riemannian metric $g_{\widetilde{X}}$, then the associated holonomy group $\operatorname{Hol}\left(\widetilde{X}, g_{\widetilde{X}}\right)$ is isomorphic to $\mathrm{SU}(2)$.

\footnotetext{
${ }^{5}$ See for example [FH91, Sect. 3.3]
} 
On the singular Kummer surface: If $\omega_{A}$ is any flat metric on $A$ induced by a constant metric from $\mathbb{C}^{n}$, then $\omega_{A}$ is invariant under the action of \pm 1 on $A$, and hence descends to a singular Ricci-flat metric $\omega_{X}$ on $X$, in the sense of Theorem 3.3. The metric $\omega_{X}$ is flat on $X_{\text {reg }}$.

Example 14.2 (Symmetric square of a K3). Let $S$ be a $K 3$ surface, and let $X:=$ $S \times S /\langle i\rangle$ where $i:\left(s_{1}, s_{2}\right) \mapsto\left(s_{2}, s_{1}\right)$. The quotient map $\gamma: S \times S \rightarrow X$ is quasiétale and Galois with group $\mathbb{Z}_{2}$. Recall from [Bea83b, Sect. 6] that the Hilbert scheme $\widetilde{X}$ parametrising zero-dimensional subschemes of length two is an irreducible holomorphic symplectic manifold and admits a birational, crepant map $\pi: \widetilde{X} \rightarrow X$. Once again, we analyse the relevant Kähler-Einstein metrics.

On the resolution: Any smooth Ricci-flat Kähler metric $\omega$ on $\widetilde{X}$ with associated Riemannian metric $g_{\widetilde{X}}$, satisfies $\operatorname{Hol}\left(\widetilde{X}, g_{\widetilde{X}}\right) \cong \operatorname{Sp}(2)$.

On the singular symmetric square: On the other hand, if $\omega_{S}$ is a Ricci-flat Kähler metric on $S$, then $\mathrm{pr}_{1}^{*} \omega_{S}+\mathrm{pr}_{2}^{*} \omega_{S}$ defines a Kähler Ricci-flat metric on $S \times S$ that descends to a singular Kähler Ricci-flat metric $\omega_{X}$ on $X$, with associated Riemannian metric $g_{X_{\text {reg }}}$. One computes ${ }^{6}$ that $\operatorname{Hol}\left(X_{\text {reg }}, g_{X_{\text {reg }}}\right)$ is an extension of $S U(2) \times S U(2)$ by $\mathbb{Z}_{2}$, hence the restricted holonomy is reducible.

14.2. Finite quotients. Singular varieties with trivial canonical class can be easily be constructed by taking quotients. For an example, consider quotient of an evendimensional Abelian variety by the involution $x \mapsto-x$ or a symmetric product of an irreducible holomorphic symplectic manifold (resp. an even dimensional Calabi-Yau manifold) as in Examples 14.1 and 14.2. However, exhibiting strongly stable singular varieties with trivial canonical class seems to require more work.

14.2.1. Quotient of Abelian varieties. We construct quotients of Abelian varieties that have the algebra of reflexive holomorphic forms of a Calabi-Yau variety or of a irreducible holomorphic symplectic variety.

Example 14.3 (A fake IHS variety with canonical singularities). This is a higher dimensional generalisation of the singular Kummer surfaces discussed in Example 14.1. Take $A$ an Abelian surface, and consider $Y:=A /\langle \pm 1\rangle$. A non-zero holomorphic two-form $\omega_{A}$ on $A$ descends to a symplectic form $\omega_{Y}$ of $Y_{\text {reg. Now, let }}$ us consider $X:=Y^{(n)}$ the $n$-th symmetric product of $Y$ for some $n \geq 2$. The variety $X$ is realised as the quotient $Y^{n} / \mathfrak{S}_{n}$. The two-form $\sum \operatorname{pr}_{i}^{*} \omega_{Y}$ is a $\mathfrak{S}_{n}$-invariant symplectic form on $\left(Y_{\text {reg }}\right)^{n}$, where $\mathrm{pr}_{i}: Y^{n} \rightarrow Y$ is the projection to the $i$-th factor.

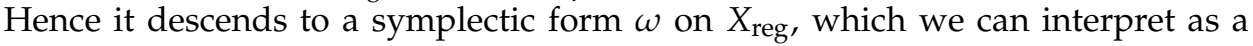
reflexive two-form on $X$. It is not hard to see that $\bigoplus_{p=0}^{2 n} H^{0}\left(X, \Omega_{X}^{[p]}\right)=\mathbb{C}[\omega]$. In particular, $X$ has the same algebra of reflexive forms as a smooth irreducible holomorphic symplectic manifold. However, its augmented irregularity is maximal, that is, equal to the dimension of $X$. The tangent sheaf $\mathscr{T}_{X_{\text {reg }}}$ is flat and $X$ admits a quasi-étale, Galois cover that is an Abelian variety.

In [Mat15], Matsushita studies what he calls cohomologically irreducible symplectic (CIHS) varieties. By definition, these are projective, holomorphic symplectic varieties $X$ satisfying the following two conditions.

- The variety $X$ has $\mathbb{Q}$-factorial, terminal singularities.

- We have an isomorphism of algebras, $\bigoplus_{p=0}^{2 n} H^{0}\left(X, \Omega_{X}^{[p]}\right)=\mathbb{C}[\omega]$, where $\omega$ is a holomorphic symplectic form.

\footnotetext{
${ }^{6}$ See Remark 4.6 and Proposition 4.3 below, as well as the first few lines of Sect. 10
} 
While these varieties share many properties with smooth irreducible holomorphic symplectic manifolds, they are not necessarily IHS, see the next example. This should be compared with the smooth case, where requiring the second condition to hold already forces the manifold to be simply connected, and hence IHS, see [GKP16b, Rem. 8.19].

Example 14.4 (A CIHS variety that has maximal augmented irregularity). Let $A$ be an Abelian surface and $t_{0} \in A$ be a two-torsion point. Let $\operatorname{tr}_{t_{0}}$ be the translation by $\left(0, t_{0}\right)$, consider the morphism $\tau:(s, t) \mapsto(t, s)$ as well as $\varphi:=\operatorname{tr}_{t_{0}} \circ \tau$. Observe that $\varphi$ induces a free action of $\mathbb{Z}_{4}$ on $A \times A$. We notice that $\varphi$ commutes with $(-1):(s, t) \mapsto(-s,-t)$, so that $\varphi$ and $(-1)$ generate an action of $G:=\mathbb{Z}_{4} \times \mathbb{Z}_{2}$ on $A \times A$. We set $X:=(A \times A) / G$ and let $\pi: A \times A \rightarrow X$ be the quotient map. As the $\mathbb{Z}_{4}$-action is free, the map $\pi$ is quasi-étale and the singularities of $X$ are exactly the images of the 256 two-torsion points under $\pi$, which are therefore isolated. Either by direct computation or by a result of Namikawa [Nam01, Cor. 1], we see that $X$ has terminal singularities, which are in addition obviously $Q$-factorial. On the other hand, by construction

$$
H^{0}\left(A \times A, \Omega_{A \times A}^{2}\right)^{G}=\mathbb{C} \cdot \omega \quad \text { where } \omega=\operatorname{pr}_{1}^{*}\left(d z_{1} \wedge d z_{2}\right)+\operatorname{pr}_{2}^{*}\left(d z_{1} \wedge d z_{2}\right) .
$$

Observe that $\omega$ is symplectic, and that $H^{0}\left(A \times A, \Omega_{A \times A}^{p}\right)^{G}=\{0\}$ for $p=1,3$.

Example 14.5 (A fake CY threefold). The Calabi-Yau case is a bit more involved, but still well-known, cf. [OS01, Ex. 2.17]. That example yields a free action of $G:=\mathbb{Z}_{2} \times \mathbb{Z}_{2}$ on a product $A=E_{1} \times E_{2} \times E_{3}$ of three elliptic curves such that

$$
H^{0}\left(A, \Omega_{A}^{p}\right)^{G}= \begin{cases}1 & \text { if } p=0,3 \\ 0 & \text { if } p=1,2\end{cases}
$$

In particular, $A / G$ is a smooth manifold with trivial canonical bundle and the algebra of holomorphic form of a Calabi-Yau threefold.

14.2.2. Quotients of $C Y$ manifolds. A classical way to produce singular $C Y$ varieties with quotient singularities is to start with a Fano manifold $X$ of dimension at least 3 and a finite group $G$ acting on $X$. Then, one considers a general element $Y \in\left|-K_{X}\right|^{G}$. If $Y$ is smooth, then it is an irreducible Calabi-Yau manifold endowed with an action of $G$. Indeed, it has trivial canonical bundle by adjunction, it is simply connected by the Lefschetz hyperplane theorem and as $X$ is Fano, one has $h^{0}\left(X, \Omega_{X}^{p}\right)=0$ for $p>0$ by Kodaira-Nakano vanishing, which in turn implies that $h^{0}\left(Y, \Omega_{Y}^{p}\right)=0$ for $0<p<\operatorname{dim} Y$ by the Lefschetz theorem for Hodge groups, see [Laz04, Lem. 4.2.2]. The variety $Y / G$ has klt singularities. Moreover, if $G$ preserves the holomorphic volume form on $Y$, then $Y / G$ has trivial canonical bundle; in particular it is Gorenstein with canonical singularities. In that case, $Y / G$ is automatically a Calabi-Yau variety in the sense of Definition 1.3.

Example 14.6 (A terminal quotient with non-Gorenstein isolated singularities). In [Fav16, Ex. 1], Favale shows that $X=\mathbb{P}^{2} \times \mathbb{P}^{2}$ admits an action of $G=\mathbb{Z}_{3}$ such that a general element $Y \in\left|-K_{X}\right|^{G}$ is smooth and such that $G$ does not preserve the holomorphic volume form on $Y$. The singular locus of the variety $Y / G$ consists of 9 points, each of them being terminal. The restricted holonomy is equal to $\mathrm{SU}(3)$, yet $Y / G$ is not $C Y$.

The following example is constructed in a similar fashion, although the details are more technical to work out. It is Gorenstein, has trivial canonical bundle, and possesses non-isolated singularities. 
Example 14.7 (A CY threefold with a one-dimensional singular locus). Now, following [Fav16, Ex. 4], take $X=\mathbb{P}^{1} \times \mathbb{P}^{1} \times \mathbb{P}^{1} \times \mathbb{P}^{1}$. It admits an action of $G=D_{16} \times \mathbb{Z}_{2}$ where $D_{16}$ is the dihedral group. As explained in loc. cit., a general element $Y \in\left|-K_{X}\right|^{G}$ is smooth and $G$ preserves the holomorphic volume form on $Y$. Moreover, the singular locus of the variety $Y / G$ has dimension one -it is not irreducible and some of its components may be zero-dimensional though.

14.2.3. Quotients of $K 3 \times K 3$ and of IHS manifolds. The following example is an example of cohomologically irreducible holomorphic symplectic variety in the sense of Matsushita [Mat15], cf. also Section 14.2.1 and Example 14.4 above, yet it is covered by a product of two K3-surfaces.

Example 14.8 (A CIHS variety with restricted holonomy $\mathrm{SU}(2) \times \mathrm{SU}(2)$ ). Let $S$ be a K3-surface with a symplectic involution $\tau$. The fixed point locus of $\tau$ consists of isolated points. Consider the action of $\mathbb{Z}_{4}$ on $S \times S$ generated by the automorphism $\sigma$ defined by $(x, y) \mapsto(\tau(y), x)$. The fixed points of $\sigma$ are of the form $(x, x)$ where $x \in \operatorname{Fix}(\tau)$, hence $X:=(S \times S) /\langle\sigma\rangle$ has isolated, Q-factorial singularities. Moreover, we have by construction

$$
\bigoplus_{p} H^{0}\left(S \times S, \Omega_{S \times S}^{p}\right)^{\langle\sigma\rangle}=\mathbb{C}[\omega] \text { where } \omega=\operatorname{pr}_{1}^{*} \omega_{S}+\operatorname{pr}_{2}^{*} \omega_{S}
$$

for some symplectic form $\omega_{S}$ on $S$. Using Namikawa's result [Nam01, Cor.1] as before, we see that $X$ has terminal singularities, and therefore it is a CHIS variety. However, it is covered by the product of two K3-surfaces, so it is not a quotient of an IHS variety.

We conclude with an example that shows that given a variety with trivial canonical bundle and restricted holonomy $\operatorname{Sp}\left(\frac{n}{2}\right)$, taking a finite, quasi-étale cover is indeed necessary before a symplectic form will necessarily exist, cf. Section 12.2 above.

Example 14.9 (A quotient of an IHS manifold with $K_{X}$ trivial but no two-form). Let $S$ be a $K 3$ surface endowed with an anti-symplectic involution $\tau$. For instance, take $S$ to be the minimal resolution of the the quotient $(E \times E) /\langle\sigma\rangle$ where $E$ is the elliptic curve $\mathbb{C} /(\mathbb{Z} \oplus i \mathbb{Z})$ and $\sigma$ acts on $E \times E$ by $\operatorname{diag}(i,-i)$. Define then $\tau$ to be the lift of $\operatorname{diag}(-1,1)$ to $S$, cf. [OZ96, Ex. 2]. Now, let us consider $S^{[2]}$ the Hilbert scheme parametrising length 2 zero-dimensional subschemes of $S$. The variety $S^{[2]}$ is an irreducible holomorphic symplectic manifold endowed with an anti-symplectic involution that we will still call $\tau$. The fixed locus of $\tau$ is a smooth Lagrangian submanifold of $S^{[2]}$. If $\omega$ is a symplectic form on $S^{[2]}$, then $\tau^{*} \omega=$ $-\omega$ hence $\tau^{*} \omega^{2}=\omega^{2}$. In particular $X:=S^{[2]} /\langle\tau\rangle$ has canonical singularities (concentrated along a surface), trivial canonical bundle, but no non-zero two-form. However, it has a quasi-étale cover that is an IHS manifold.

14.3. Moduli spaces of sheaves on K3-surfaces. Let $S$ be projective K3-surface. As usual, we equip the even integral cohomology of $S$ with the pairing

$$
\langle v, w\rangle:=-\int_{X} v w^{*},
$$

where $w^{*}=(-1)^{i} w$ for $w \in H^{2 i}(S, \mathbb{Z})$. To each coherent sheaf $E$ we associate its Mukai vector $v(E)=\operatorname{ch}(E) \sqrt{\operatorname{td}(S)} \in H^{\text {even }}(S, \mathbb{Z})$. For a given $v$ and an ample Cartier divisor $H$ on $S$, we denote by $M_{v}(H)$ the Gieseker-Maruyama moduli space of $H$-semistable sheaves with Mukai vector $v$ on $S$. Any given $v$ can be decomposed as $v=m v_{0}$, where $v_{0}=\left(r_{0}, c_{0}, a_{0}\right)$ is primitive and $m \in \mathbb{N}^{+}$. For simplicity, we assume that $r_{0}>0$ in the following. Under the additional assumption 
that $H$ is "v-general", every $H$-semistable sheaf is $H$-stable, and the corresponding moduli space is non-empty if and only if $c_{0} \in \mathrm{NS}(S)$ and $\left\langle v_{0}, v_{0}\right\rangle \geq-2$. In [KLS06], Kaledin, Lehn, and Sorger show that if either $m \geq 2$ and $\left\langle v_{0}, v_{0}\right\rangle>2$, or $m>2$ and $\left\langle v_{0}, v_{0}\right\rangle \geq 2$, then $M_{m v_{0}}(H)$ is a projective variety with locally factorial, symplectic (and hence canonical) singularities that admits a holomorphic symplectic two-form, but no symplectic resolution. Assuming that $\left\langle v_{0}, v_{0}\right\rangle \geq 2$ and $m \geq 1$, Perego and Rapagnetta recently proved in [PR18, Thm. 1.19] that $M_{m v_{0}}(H)$ is an IHS-variety. They also prove a similar statement for moduli of sheaves on abelian surfaces.

\section{REFERENCES}

[Ara02] Donu Arapura. Higgs bundles, integrability, and holomorphic forms. In Motives, polylogarithms and Hodge theory, Part II (Irvine, CA, 1998), volume 3 of Int. Press Lect. Ser., pages 605-624. Int. Press, Somerville, MA, 2002. $\uparrow 48$

[BCHM10] Caucher Birkar, Paolo Cascini, Christopher D. Hacon, and James McKernan. Existence of minimal models for varieties of log general type. J. Amer. Math. Soc., 23:405-468, 2010. DOI:10.1090/S0894-0347-09-00649-3. ^26

[Bea83a] Arnaud Beauville. Some remarks on Kähler manifolds with $c_{1}=0$. In Classification of algebraic and analytic manifolds (Katata, 1982), volume 39 of Progr. Math., pages 1-26. Birkhäuser Boston, Boston, MA, 1983. $\uparrow 30$

[Bea83b] Arnaud Beauville. Variétés Kähleriennes dont la première classe de Chern est nulle. J. Differential Geom., 18(4):755-782 (1984), 1983. euclid.jdg/1214438181. ^2, 47, 50

[BEG13] Sébastien Boucksom, Philippe Eyssidieux, and Vincent Guedj, editors. An introduction to the Kähler-Ricci flow, volume 2086 of Lecture Notes in Mathematics. Springer, 2013. DOI:10.1007/978-3-319-00819-6. $\uparrow 12$

[Ber94] A. Jon Berrick. Groups with no nontrivial linear representations. Bull. Austral. Math. Soc., 50(1):1-11, 1994. DOI:10.1017/S0004972700009503. $\uparrow 47$

[Bes87] Arthur L. Besse. Einstein manifolds, volume 10 of Ergebnisse der Mathematik und ihrer Grenzgebiete (3) [Results in Mathematics and Related Areas (3)]. Springer-Verlag, Berlin, 1987. DOI:10.1007/978-3-540-74311-8. ^8, 9, 15, 18, 19, 27, 28

[BK08] V. Balaji and János Kollár. Holonomy groups of stable vector bundles. Publ. Res. Inst. Math. Sci., 44(2):183-211, 2008. DOI:10.2977/prims/1210167326. $\uparrow 41,44,45,46$

[BKT13] Yohan Brunebarbe, Bruno Klingler, and Burt Totaro. Symmetric differentials and the fundamental group. Duke Math. J., 162(14):2797-2813, 2013. DOI:10.1215/00127094-2381442. $\uparrow 6,48$

[BtD85] Theodor Bröcker and Tammo tom Dieck. Representations of compact Lie groups, volume 98 of Graduate Texts in Mathematics. Springer-Verlag, New York, 1985. DOI:10.1007/978-3-662-12918-0. $\uparrow 40$

[Bum04] Daniel Bump. Lie groups, volume 225 of Graduate Texts in Mathematics. Springer-Verlag, New York, 2004. DOI:10.1007/978-1-4757-4094-3. ^38

[Cam91] Frédéric Campana. On twistor spaces of the class $\mathscr{C}$. J. Differential Geom., 33(2):541-549, 1991. euclid.jdg/1214446329. $\uparrow 46$

[Cam95] Frédéric Campana. Fundamental group and positivity of cotangent bundles of compact Kähler manifolds. J. Algebraic Geom., 4(3):487-502, 1995. ^ 6, 46, 47

[CGP13] Frédéric Campana, Henri Guenancia, and Mihai Păun. Metrics with cone singularities along normal crossing divisors and holomorphic tensor fields. Ann. Sci. Éc. Norm. Supér. (4), 46(6):879-916, 2013. $\uparrow 32,33$

[CL06] Paolo Cascini and Gabriele La Nave. Kähler-Ricci Flow and the Minimal Model Program for Projective Varieties. Preprint arXiv:0603064., 2006. $\uparrow 11$

[Dem85] Jean-Pierre Demailly. Mesures de Monge-Ampère et caractérisation géométrique des variétés algébriques affines. Mém. Soc. Math. France (N.S.), 19:124, 1985. 12

[Dem12] Jean-Pierre Demailly. Complex Analytic and Differential Geometry, June 2012. OpenContent Book, freely available from the author's web site, http://www-fourier.ujf-grenoble.fr/ demailly/books.html. $\uparrow 34,38$

[DG94] Gerd Dethloff and Hans Grauert. Seminormal complex spaces. In Several Complex Variables VII, volume 74 of Encyclopaedia Math. Sci., pages 183-220. Springer, Berlin, 1994. $\uparrow 10$

[DP10] Jean-Pierre Demailly and Nefton Pali. Degenerate complex Monge-Ampère equations over compact Kähler manifolds. Internat. J. Math., 21(3):357-405, 2010. $\uparrow 11$

[Dru18] Stéphane Druel. A decomposition theorem for singular spaces with trivial canonical class of dimension at most five. Invent. Math., 211(1):245-296, 2018. 


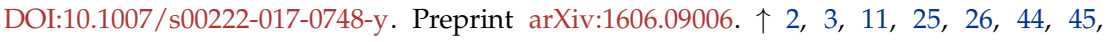
46

[EGZ09] Philippe Eyssidieux, Vincent Guedj, and Ahmed Zeriahi. Singular Kähler-Einstein metrics. J. Amer. Math. Soc., 22(3):607-639, 2009. DOI:10.1090/S0894-0347-09-00629-8. 个 2, 3, 4, 11, $12,13,32$

[Fav16] Filippo Favale. Calabi-Yau quotients with terminal singularities. Preprint arXiv:1306.3555., 2016. $\uparrow 51,52$

[FH91] William Fulton and Joe Harris. Representation theory. A first course, volume 129 of Graduate Texts in Mathematics. Springer-Verlag, New York, 1991. DOI:10.1007/978-1-4612-0979-9. $\uparrow 46,49$

[FL81] William Fulton and Robert Lazarsfeld. Connectivity and its applications in algebraic geometry. In Algebraic geometry (Chicago, Ill., 1980), volume 862 of Lecture Notes in Math., pages 26-92. Springer, Berlin, 1981. DOI:10.1007/BFb0090889. $\uparrow 6$

[GKKP11] Daniel Greb, Stefan Kebekus, Sándor J. Kovács, and Thomas Peternell. Differential forms on log canonical spaces. Inst. Hautes Études Sci. Publ. Math., 114(1):87-169, November 2011. DOI:10.1007/s10240-011-0036-0 An extended version with additional graphics is available as arXiv:1003.2913. $\uparrow 26$

[GKP14] Daniel Greb, Stefan Kebekus, and Thomas Peternell. Reflexive differential forms on singular spaces. Geometry and cohomology. J. Reine Angew. Math., 697:57-89, 2014. DOI:10.1515/crelle-2012-0097. Preprint arXiv:1202.3243. $\uparrow 38,42$

[GKP16a] Daniel Greb, Stefan Kebekus, and Thomas Peternell. Movable curves and semistable sheaves. Int. Math. Res. Not., 2016(2):536-570, 2016. DOI:10.1093/imrn/rnv126. Preprint arXiv:1408.4308. $\uparrow 22$

[GKP16b] Daniel Greb, Stefan Kebekus, and Thomas Peternell. Singular spaces with trivial canonical class. In Minimal Models and Extremal Rays, Kyoto, 2011, volume 70 of Adv. Stud. Pure Math., pages 67-113. Mathematical Society of Japan, Tokyo, 2016. Preprint arXiv:1110.5250. $\uparrow 2,4$, $5,11,19,22,24,25,29,42,43,46,47,51$

[GKP16c] Daniel Greb, Stefan Kebekus, and Thomas Peternell. Étale fundamental groups of Kawamata log terminal spaces, flat sheaves, and quotients of abelian varieties. Duke Math. J., 165(10):1965-2004, 2016. DOI:10.1215/00127094-3450859. Preprint arXiv:1307.5718. $\uparrow 3$, $24,26,27,39,48$

[Gra16] Patrick Graf. A Mehta-Ramanathan theorem for linear systems with basepoints. Math. Nachr., 289(10):1208-1218, 2016. DOI:10.1002/mana.201500180. Preprint arXiv:1501.04210. $\uparrow 44$

[Gro70] Alexander Grothendieck. Représentations linéaires et compactification profinie des groupes discrets. Manuscripta Math., 2:375-396, 1970. DOI:10.1007/BF01719593. $\uparrow 48$

[Gro71] Alexandre Grothendieck. Revêtements étales et groupe fondamental (SGA 1). Springer-Verlag, Berlin, 1971. Séminaire de Géométrie Algébrique du Bois Marie 1960-1961, Dirigé par Alexandre Grothendieck. Augmenté de deux exposés de Michèle Raynaud, Lecture Notes in Mathematics, Vol. 224. Also available as arXiv:math/0206203. $\uparrow 10$

[Gue16] Henri Guenancia. Semistability of the tangent sheaf of singular varieties. Algebraic Geometry, 3(5):508-542, November 2016. DOI:10.14231/AG-2016-024. Preprint arXiv:1502.03711. 个 3, 4, 7, 19, 21, 22, 26, 29, 31, 32, 33, 34, 35

[Gun90] Robert C. Gunning. Introduction to holomorphic functions of several variables. Vol. III. Homological theory. The Wadsworth \& Brooks/Cole Mathematics Series. Wadsworth \& Brooks/Cole Advanced Books \& Software, Monterey, CA, 1990. $\uparrow 28$

[GW09] Roe Goodman and Nolan R. Wallach. Symmetry, representations, and invariants, volume 255 of Graduate Texts in Mathematics. Springer, Dordrecht, 2009. DOI:10.1007/978-0-387-79852-3. $\uparrow 42,46$

[GZ07] Vincent Guedj and Ahmed Zeriahi. The weighted Monge-Ampère energy of quasiplurisubharmonic functions. J. Funct. Anal., 250(2):442-482, 2007. DOI:10.1016/j.jfa.2007.04.018. $\uparrow 32$

[Har77] Robin Hartshorne. Algebraic geometry. Springer-Verlag, New York, 1977. Graduate Texts in Mathematics, No. 52. DOI:10.1007/978-1-4757-3849-0. 77

[HL10] Daniel Huybrechts and Manfred Lehn. The geometry of moduli spaces of sheaves. Cambridge Mathematical Library. Cambridge University Press, Cambridge, second edition, 2010. DOI:10.1017/CBO9780511711985. $\uparrow 26$

[HP17] Andreas Höring and Thomas Peternell. Algebraic integrability of foliations with numerically trivial canonical bundle. Preprint arXiv:1710.06183, October 2017. $\uparrow 2,4$

[Huy05] Daniel Huybrechts. Complex geometry. Universitext. Springer-Verlag, Berlin, 2005. DOI:10.1007/b137952. $\uparrow 8$

[Joy00] Dominic D. Joyce. Compact manifolds with special holonomy. Oxford Mathematical Monographs. Oxford University Press, Oxford, 2000. $\uparrow 8$ 
[Kle10] Bruce Kleiner. A new proof of Gromov's theorem on groups of polynomial growth. J. Amer. Math. Soc., 23(3):815-829, 2010. DOI:10.1090/S0894-0347-09-00658-4. 个 47, 48

[Kli13] Bruno Klingler. Symmetric differentials, Kähler groups and ball quotients. Invent. Math., 192(2):257-286, 2013. DOI:10.1007/s00222-012-0411-6. $\uparrow 48$

[KLS06] Dmitry Kaledin, Manfred Lehn, and Christoph Sorger. Singular symplectic moduli spaces. Invent. Math., 164(3):591-614, 2006. DOI:10.1007/s00222-005-0484-6. ^53

[KM98] János Kollár and Shigefumi Mori. Birational geometry of algebraic varieties, volume 134 of Cambridge Tracts in Mathematics. Cambridge University Press, Cambridge, 1998. With the collaboration of C. H. Clemens and A. Corti, Translated from the 1998 Japanese original. DOI:10.1017/CBO9780511662560. $\uparrow 7,10,11$

[KN96a] Shoshichi Kobayashi and Katsumi Nomizu. Foundations of differential geometry. Vol. I. Wiley Classics Library. John Wiley \& Sons, Inc., New York, 1996. Reprint of the 1963 original. $\uparrow 9$, 15

[KN96b] Shoshichi Kobayashi and Katsumi Nomizu. Foundations of differential geometry. Vol. II. Wiley Classics Library. John Wiley \& Sons, Inc., New York, 1996. Reprint of the 1969 original. $\uparrow 9$

[Kob80] Shoshichi Kobayashi. The first Chern class and holomorphic symmetric tensor fields. J. Math. Soc. Japan, 32(2):325-329, 1980. DOI:10.2969/jmsj/03220325. 39

[Kob87] Shoshichi Kobayashi. Differential geometry of complex vector bundles, volume 15 of Publications of the Mathematical Society of Japan. Iwanami Shoten and Princeton University Press, Princeton, NJ, 1987. Kanô Memorial Lectures, 5. $\uparrow 17,34$

[Ko195] János Kollár. Shafarevich maps and automorphic forms. M. B. Porter Lectures. Princeton University Press, Princeton, NJ, 1995. $\uparrow 46$

[Koł98] Sławomir Kołodziej. The complex Monge-Ampère equation. Acta Math., 180(1):69-117, 1998. DOI:10.1007/BF02392879. $\uparrow 33$

[Laz04] Robert Lazarsfeld. Positivity in algebraic geometry. I. Classical setting: line bundles and linear series, volume 48 of Ergebnisse der Mathematik und ihrer Grenzgebiete. 3. Folge. SpringerVerlag, Berlin, 2004. DOI:10.1007/978-3-642-18808-4. $\uparrow 11,25,51$

[Mat15] Daisuke Matsushita. On base manifolds of Lagrangian fibrations. Sci. China Math., 58(3):531-542, 2015. DOI:10.1007/s11425-014-4927-7. ^50, 52

[Nak04] Noboru Nakayama. Zariski-decomposition and abundance, volume 14 of MSJ Memoirs. Mathematical Society of Japan, Tokyo, 2004. $\uparrow 11$

[Nam01] Yoshinori Namikawa. A note on symplectic singularities. preprint, arXiv:math/0101028, 2001. $\uparrow 51,52$

[OS01] Keiji Oguiso and Jun Sakurai. Calabi-Yau threefolds of quotient type. Asian J. Math., 5(1):43-77, 2001. DOI:10.4310/AJM.2001.v5.n1.a5. $\uparrow 40,51$

[OS11] Keiji Oguiso and Stefan Schröer. Enriques manifolds. J. Reine Angew. Math., 661:215-235, 2011. DOI:10.1515/CRELLE.2011.077. 447

[OZ96] Keiji Oguiso and De-Qi Zhang. On the most algebraic K3 surfaces and the most extremal log Enriques surfaces. Amer. J. Math., 118(6):1277-1297, 1996. DOI:10.1353/ajm.1996.0052. $\uparrow 52$

[Pău08] Mihai Păun. Regularity properties of the degenerate Monge-Ampère equations on compact Kähler manifolds. Chin. Ann. Math., Ser. B, 29(6):623-630, 2008. 111

[Pet94] Thomas Peternell. Minimal varieties with trivial canonical classes. I. Math. Z., 217(3):377405, 1994. DOI:10.1007/BF02571950. $\uparrow 30$

[PR18] Arvid Perego and Antonio Rapagnetta. The moduli spaces of sheaves on K3 surfaces are irreducible symplectic varieties. Preprint arXiv:1802.01182., February 2018. 553

[RZ11] Xiaochun Rong and Yuguang Zhang. Continuity of extremal transitions and flops for Calabi-Yau manifolds. J. Differential Geom., 89(2):233-269, 2011. Appendix B by Mark Gross. euclid.jdg/1324477411. $\uparrow 15$

[Sim62] James Simons. On the transitivity of holonomy systems. Ann. of Math. (2), 76:213-234, 1962. $\uparrow 19$

[SY94] Richard M. Schoen and Shing-Tung Yau. Lectures on differential geometry. Conference Proceedings and Lecture Notes in Geometry and Topology, I. International Press, Cambridge, MA, 1994. $\uparrow 15$

[Tak03] Shigeharu Takayama. Local simple connectedness of resolutions of log-terminal singularities. Internat. J. Math., 14(8):825-836, 2003. DOI:10.1142/S0129167X0300196X. ^47, 48

[Tia96] Gang Tian. Kähler-Einstein metrics on algebraic manifolds. In Transcendental methods in algebraic geometry (Cetraro, 1994), volume 1646 of Lecture Notes in Math., pages 143-185. Springer, Berlin, 1996. DOI:10.1007/BFb0094304. $\uparrow 39$

[Tos09] Valentino Tosatti. Limits of Calabi-Yau metrics when the Kähler class degenerates. J. Eur. Math. Soc. (JEMS), 11(4):755-776, 2009. DOI:b10.4171/JEMS/165. Preprint arXiv:0710.4579. $\uparrow 15$ 
[Tsu88] Hajime Tsuji. Existence and degeneration of kähler-einstein metrics on minimal algebraic varieties of general type. Math. Ann., 281(1):123-133, 1988. DOI:10.1007/BF01449219. $\uparrow 11$

[TZ06] Gang Tian and Zhou Zhang. On the Kähler-Ricci flow on projective manifolds of general type. Chinese Ann. Math. Ser. B, 27(2):179-192, 2006. DOI:10.1007/s11401-005-0533-x. 111

[Yau78] Shing-Tung Yau. On the Ricci curvature of a compact Kähler manifold and the complex Monge-Ampère equation. I. Comm. Pure Appl. Math., 31(3):339-411, 1978. DOI:10.1002/cpa.3160310304. $\uparrow 32$

[Zha06] Zhou Zhang. On degenerate Monge-Ampère equations over closed Kähler manifolds. Int. Math. Res. Not., pages 1-18, 2006. DOI:10.1155/IMRN/2006/63640. 11

DANIEL Greb, ESSENER Seminar FÜr Algebraische GeOMETRIE Und ARITHMEtiK, FAKUltät FÜr MATHEMATIK, UNIVERSITÄT DUISBURG-ESSEN, 45117 ESSEN, GERMANY

E-mail address: daniel.greb@uni-due.de

URL: http://www.esaga.uni-due.de/daniel.greb

Henri Guenancia, Department of Mathematics, Stony Brook University, Stony BROOK, NY 11794-3651, U.S.A.

E-mail address: henri.guenancia@stonybrook.edu

URL: http://www . math. stonybrook. edu/ guenancia

Stefan Kebekus, Mathematisches Institut, Albert-Ludwigs-Universität Freiburg, ERNST-ZERMELO-STRASSE 1, 79104 FREIBURG IM BREISGAU, GERMANY \& FREIBURG INSTITUTE FOR ADVANCED STUdies (FRIAS), FREIBURG IM BREISGAU, GERMANY \& UNIVERSITY OF STRASBOURG INSTITUTE FOR ADVANCED STUDY (USIAS), STRASBOURG, FRANCE

E-mail address: stef an .kebekus@math. uni-freiburg. de URL: https://cplx.vm.uni-freiburg.de 